\title{
A geometric recipe for twisted superpotentials
}

\author{
Lotte Hollands, ${ }^{a, b, c}$ Philipp Rüter $^{a, b}$ and Richard J. Szabo ${ }^{a, b, c}$ \\ ${ }^{a}$ Department of Mathematics, Heriot-Watt University, \\ Edinburgh Campus, Edinburgh, U.K. \\ ${ }^{b}$ Maxwell Institute for Mathematical Sciences, \\ Bayes Centre, Edinburgh, U.K. \\ ${ }^{c}$ Higgs Centre for Theoretical Physics, \\ James Clerk Maxwell Building, Edinburgh, U.K. \\ E-mail: 1.hollands@hw.ac.uk, pr26@hw.ac.uk, r.j.szabo@hw.ac.uk
}

ABSTRACT: We give a pedagogical introduction to spectral networks and abelianization, as well as their relevance to $\mathcal{N}=2$ supersymmetric field theories in four dimensions. Motivated by a conjecture of Nekrasov-Rosly-Shatashvili, we detail a geometric recipe for computing the effective twisted superpotential for $\mathcal{N}=2$ field theories of class $\mathcal{S}$ as a generating function of the brane of opers, with respect to the spectral coordinates found from abelianization. We present two new examples, the simplest Argyres-Douglas theory and the pure $\mathrm{SU}(2)$ gauge theory, while we conjecture the $\epsilon$-expansion of the effective twisted superpotential for the $E_{6}$ Minahan-Nemeschansky theory.

Keywords: Differential and Algebraic Geometry, Duality in Gauge Field Theories, Supersymmetric Gauge Theory

ARXIV EPRINT: 2109.14699 


\section{Contents}

1 Introduction 1

1.1 Background 2

1.2 Summary and outline 3

1.3 Open questions 4

2 Spectral networks and abelianization $\quad 6$

2.1 WKB spectral networks 6

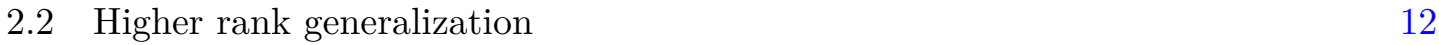

2.3 Abelianization 14

$\begin{array}{lll}2.4 & \text { Spectral coordinates } & 19\end{array}$

2.5 Nonabelianization 24

3 Theories of class $\mathcal{S}$ and their BPS states $\quad 26$

3.1 Seiberg-Witten geometry 26

$\begin{array}{lll}3.2 & \text { Class } \mathcal{S} \text { geometry } & 30\end{array}$

3.3 BPS states from spectral networks 36

4 Twisted superpotentials, Hitchin systems and opers 43

4.1 Effective twisted superpotentials 43

4.2 Hitchin systems 46

4.3 Opers 49

4.4 Exact WKB analysis 53

5 The Nekrasov-Rosly-Shatashvili correspondence $\quad 61$

5.1 The NRS conjecture $\quad 62$

5.2 A geometric recipe 63

$5.3 \quad \mathrm{AD}_{2}$ theory 66

$\begin{array}{lll}5.4 & \text { Pure } \mathrm{SU}(2) \text { theory } & 68\end{array}$

$\begin{array}{lll}5.5 & E_{6} \text { Minahan-Nemeschansky theory } & 75\end{array}$

$\begin{array}{ll}5.6 & \text { Derivation from quantum field theory }\end{array}$

$\begin{array}{lll}5.7 & \text { A more general effective twisted superpotential } & 79\end{array}$

\section{Introduction}

In these notes we give a pedagogical introduction to four-dimensional $\mathcal{N}=2$ supersymmetric field theories of class $\mathcal{S}$ and certain novel geometric methods that can be used to study them. 


\subsection{Background}

The origin of class $\mathcal{S}$ theories lies in six dimensions in the $\mathcal{N}=(2,0)$ superconformal field theory with gauge algebra $\mathfrak{g}$. This theory is rather mysterious and elusive: it does not admit a Lagrangian description and it might not even exist as a classical theory. ${ }^{1}$ However, it can be studied indirectly after compactification on lower-dimensional submanifolds where it gives rise to a host of new structures in both quantum field theory and geometry. In fact, it has broadened our fundamental understanding of what a quantum field theory really is.

The first evidence for the six-dimensional $(2,0)$-theory traces back to the seminal work by Nahm in his classification of the simple Lie superalgebras which can occur as the symmetry algebra of a superconformal quantum field theory. The highest dimensions in which a superconformal field theory can exist was found to be six with the superconformal algebra $\mathfrak{o s p}(2,6 \mid 2 k)$; the theory with the highest amount of supercharges in six dimensions is the $\mathcal{N}=(2,0)$ theory [107]. At the time there was no known way to actually construct this theory, but later on constructions of this six-dimensional superconformal field theory in terms of string theory were realized independently by Witten [141] and Strominger [129].

One way to construct this theory is in type IIB string theory on an ADE singularity. This is defined in the ten-dimensional spacetime which is the direct product of $\mathbb{R}^{5,1}$ with the resolution of an orbifold singularity. An orbifold singularity is a quotient $\mathbb{C}^{2} / \Gamma$ by the natural action of a finite subgroup $\Gamma \subset \mathrm{SU}(2)$ (with $\mathbb{C}^{2}$ viewed as the fundamental representation of $\mathrm{SU}(2))$. By the McKay correspondence, $\Gamma$ can be identified with the Dynkin diagram of type ADE of a simply-laced Lie algebra $\mathfrak{g}=\mathfrak{g}(\Gamma)$. By wrapping a D3-brane on a vanishing two-cycle of the resolution of $\mathbb{C}^{2} / \Gamma$, the localized dynamics at the tip $0 \in \mathbb{C}^{2} / \Gamma$ describes Nahm's six-dimensional superconformal field theory. The resulting theory on $\mathbb{R}^{5,1}$ is labeled by the corresponding Lie algebra as $\mathfrak{X}=\mathfrak{X}[\mathfrak{g}]$.

The theory $\mathfrak{X}$ also has a realization in M-theory as the worldvolume theory of M5branes. Consider a collection of M5-branes, whose interactions are mediated by open M2branes ending on them. The $(2,0)$-theory is then realized in the limit where the M5-branes coincide; for instance, $K$ flat coincident M5-branes correspond to the $(2,0)$-theory $\mathfrak{X}[K]$ at an $A_{K-1}$-singularity (with $\mathfrak{g}=\mathfrak{s u}(K)$ ). For further reading about the six-dimensional $(2,0)$-theory we suggest [106].

The $(2,0)$-theory can be compactified on a (possibly punctured) Riemann surface $C$. In these notes we will study the theory $\mathfrak{X}[\mathfrak{g}]$ on the six-dimensional backgrounds

$$
C \times \mathbb{R}^{3,1} .
$$

After a partial topological twist of the theory, in the limit where $C$ collapses to a point this leads to a four-dimensional $\mathcal{N}=2$ supersymmetric field theory on $\mathbb{R}^{3,1}$ which depends only on the complex structure of the punctured Riemann surface $C$, together with certain specified singularity data $\mathcal{D}$ at the punctures of $C$. We denote this theory by $\mathrm{T}_{\mathfrak{g}}[C, \mathcal{D}]$, or simply by $\mathrm{T}$. This is called a theory of class $\mathcal{S}[54,59] .^{2}$

\footnotetext{
${ }^{1}$ It is possible to find the field content and a Lagrangian description in the specific case of the abelian gauge algebra $\mathfrak{g}=\mathfrak{u}(1)$ [33].

${ }^{2}$ The $\mathcal{S}$ stands for 'six', or alternatively for 'S-duality' which acts in a particularly nice way on the Riemann surface $C$ [132].
} 
Class $\mathcal{S}$ theories can be studied from the perspective offered by the geometry of the Riemann surface $C$, and this is the perspective we will take in these notes. One advantage of such a geometric approach is that it is not only useful for understanding the conventional four-dimensional field theories, where a Lagrangian description allows the usual methods of quantum field theory to be employed, but also for more unconventional theories where a Lagrangian description is not available and other methods fail. The theories $\mathrm{T}$ of class $\mathcal{S}$ naturally encompass both types of field theories.

\subsection{Summary and outline}

The goal of these notes is to define and compute the effective twisted superpotential for any four-dimensional $\mathcal{N}=2$ theory $\mathrm{T}$ of class $\mathcal{S}$ in the $\frac{1}{2} \Omega$-background, following a conjecture by Nekrasov, Rosly and Shatashvili [114]. ${ }^{3}$ The two main ingredients are the "brane of opers", which is a holomorphic Lagrangian submanifold in the moduli space of complexified flat connections on $C$, and a suitable system $\left\{x_{i}, y^{i}\right\}$ of holomorphic Darboux coordinates on this moduli space. With these choices the effective twisted superpotential $\widetilde{\mathcal{W}}^{\text {eff }}$ may be extracted as the generating function

$$
y^{i}=\frac{1}{\epsilon} \frac{\partial \widetilde{\mathcal{W}}^{\mathrm{eff}}(x ; \epsilon)}{\partial x_{i}}
$$

of the brane of opers with respect to the Darboux coordinates $\left\{x_{i}, y^{i}\right\}$, where $\epsilon$ is the deformation parameter of the $\frac{1}{2} \Omega$-background.

These notes have grown out of a series of lectures by the first author at the PreStringMath Summer School 2017. Paralleling these lectures, we have tried to keep these notes accessible and self-contained by including extensive background material in the first few sections. In particular, we define spectral networks on the Riemann surface $C$ and point out how they encode the four-dimensional BPS particle spectrum of the theory $T$. We introduce the central ideas of Seiberg-Witten theory and motivate the importance of $\mathcal{N}=2$ theories of class $\mathcal{S}$. We also review the abelianization method for constructing Darboux coordinate systems on the moduli space of complexified flat connections on $C$, which we relate to the exact WKB method as well as TBA-like equations. Along the way we introduce the Hitchin system and flat oper connections. All of these elements play an important role in reaching our final goal: a geometric recipe for computing the effective twisted superpotential $\widetilde{\mathcal{W}}^{\text {eff }}$ for any theory $\mathrm{T}$ of class $\mathcal{S}$.

These notes are naturally structured in four parts. Most of our new results are part of section 5 , and the expert reader may wish to skip over the earlier sections.

In section 2 we present some of the geometry, introducing (Fenchel-Nielsen type) spectral networks, abelianization and (Fenchel-Nielsen type) spectral coordinates, while emphasizing their generalization to Riemann surfaces $C$ which have irregular punctures. Throughout we illustrate all of the formalism with many explicit examples (which will be useful in section 5).

\footnotetext{
${ }^{3}$ For readers more familiar with topological string theory, we mention that this superpotential equals the refined topological string free energy $\mathcal{F}^{\mathrm{NS}}$ in the two-dimensional Nekrasov-Shatashvili limit.
} 
In section 3 we move on to the physics side of the story. After a brief introduction to Seiberg-Witten theory, we introduce four-dimensional $\mathcal{N}=2$ theories of class $\mathcal{S}$ and, in particular, our main examples. These are the simplest Argyres-Douglas theory, the pure $\mathrm{SU}(2)$ gauge theory, the conformal $\mathrm{SU}(2)$ gauge theory coupled to four fundamental hypermultiplets, and the $E_{6}$ Minahan-Nemeschansky theory. As one application of the theory of spectral networks, we review how to determine the BPS particle spectrum of any theory of class $\mathcal{S}$.

In section 4 , we combine the geometry and physics perspectives through the richness of the Hitchin system. We introduce the $\frac{1}{2} \Omega$-background, along with the effective twisted superpotential of an $\mathcal{N}=2$ theory of class $\mathcal{S}$ in this background. We further motivate the brane of opers as a quantization of the Coulomb branch of the $\mathcal{N}=2$ theory, while we relate the abelianization method, applied to flat oper connections, to the exact WKB method. This shows that the spectral coordinates from section 2 have good WKB asymptotics when evaluated on flat oper connections.

In section 5, we explain the Nekrasov-Rosly-Shatashvili conjecture which relates the generating function of opers in Fenchel-Nielsen type coordinates to the effective twisted superpotential of an $\mathcal{N}=2$ theory of class $\mathcal{S}$ in the $\frac{1}{2} \Omega$-background. We then formulate our geometric recipe, and test it by computing the effective twisted superpotential $\widetilde{\mathcal{W}}^{\text {eff }}(x ; \epsilon)$ - in a perturbative expansion in the coupling or ultraviolet scale, which is exact in $\epsilon$ - in two new examples: the simplest Argyres-Douglas theory and the pure $\mathrm{SU}(2)$ gauge theory; for the treatment of the conformal $\mathrm{SU}(2)$ theory we refer to [74].

For the pure $\mathrm{SU}(2)$ theory this requires computing the asymptotically small solutions of the Mathieu equation in terms of the Mathieu functions; we are able to do this in a perturbation expansion in the ultraviolet scale, which is exact in $\epsilon$, following the approach of [74] for the Heun oper. For the $E_{6}$ Minahan-Nemeschansky theory, we content ourselves with conjecturing the $\epsilon$-expansion of the effective twisted superpotential. We conclude by describing the string theory magic behind the geometric recipe, and by giving an interpretation to the generating function of opers computed with respect to other types of spectral coordinates.

In the time elapsed between delivering and writing up these lecture notes, various other relevant works have appeared. We have tried to incorporate them into these notes, either in the main text or in section 1.3 below. Our presentation in these notes is distinguished by deriving the familiar effective twisted superpotential from scratch, without using any ingredients that are not known for instrinsically strongly coupled theories (such as the Matone relation [100]). In particular, for the weakly coupled pure $\mathrm{SU}(2)$ gauge theory we simply obtain the superpotential from the expression

$$
\widetilde{\mathcal{W}}^{\text {eff }}=\frac{1}{2} \int \mathrm{d} x \log \frac{\operatorname{Wr}\left(\psi_{1}^{\prime}, \psi_{1}^{\prime \prime}\right)}{\operatorname{Wr}\left(\psi_{1}, \psi_{1}^{\prime \prime}\right)} \frac{\operatorname{Wr}\left(\psi_{1}, \psi_{2}^{\prime \prime}\right)}{\operatorname{Wr}\left(\psi_{1}^{\prime}, \psi_{2}^{\prime \prime}\right)},
$$

where Wr denotes the Wronskian and the $\psi$ 's are certain solutions to the Mathieu equation (5.24); see section 5.4 for details.

\subsection{Open questions}

These notes do not by any means close the door on this topic. There are various open computational as well as conceptual questions to be addressed. Let us mention a few here: 
- Can one extend the computations of the effective twisted superpotential for the pure $\mathrm{SU}(2)$ theory to higher order, or perhaps even to all orders?

- Is it possible to find the exact form of the effective twisted superpotential for the $E_{6}$ Minahan-Nemeshansky theory? Can one verify its relation to the corresponding topological string partition function?

- There are many more interesting examples to apply the recipe to, for instance more general Argyres-Douglas theories. Is there some new physics encoded in the corresponding effective twisted superpotentials?

- Is it possible to find a proof of the geometric recipe (in one of the examples or even in general), perhaps taking inspiration from [85]?

- Can one develop a better physical framework for studying the relation between $\mathcal{N}=2$ boundary conditions and spectral coordinates?

- Any flat connection $\nabla$ can be rewritten as an oper connection, possibly with apparent singularities. In these notes we only discuss the generating function for the brane of opers without apparent singularities. Can one define more general generating functions? What is the physical relevance of these? (This may compute the $\mathcal{N}=2$ superpotential of an $\mathcal{N}=2$ theory with added surface defects.)

- Can one lift the geometric recipe to three dimensions, to compute the effective twisted superpotentials of five-dimensional $\mathcal{N}=1$ theories in the background $\mathbb{R}_{\epsilon}^{2} \times \mathbb{R}^{2} \times S^{1}$ ?

- Could we also extend the recipe to four dimensions, to compute the full Nekrasov instanton partition function of the $\mathcal{N}=2$ theory in the $\Omega$-background $\mathbb{R}_{\epsilon_{1}}^{2} \times \mathbb{R}_{\epsilon_{2}}^{2}$ (see [136] for some initial results)? And likewise to five dimensions?

- In $[35,36]$ the dual topological string (or free fermion) partition function in the self-dual $\Omega$-background $\epsilon_{1}=-\epsilon_{2}=\epsilon$ is characterized as an isomonodromic taufunction with respect to the same types of spectral coordinates, found using either abelianization or the exact WKB method. Can we understand this result from the perspective of the present paper? Aside from the difference between the self-dual and Nekrasov-Shatashvili limits, the isomonodromic tau-function is formulated with respect to opers with apparent singularities. A gauge theoretic understanding of this relation has been recently found in [86, 112], see also [23] for earlier work in this direction.

- Equally intriguing are the non-perturbative approaches to topological string theory formulated in $[34,67,80]$, which are centred around the spectral determinant of the five-dimensional mirror curve. This spectral determinant is compared to the exact WKB method for the four-dimensional pure $\mathrm{SU}(2)$ theory in [65], and for the $\mathrm{SU}(2)$ theory with one flavour in [66], in the $\frac{1}{2} \Omega$-background. What is the role of the spectral determinant in our set-up? 
- Finally, another closely related avenue should be the mathematical programme outlined in [18, 25-27], which studies a class of Riemann-Hilbert problems arising in Donaldson-Thomas theory (inspired by $[56,59]) .{ }^{4}$ This theory is worked out in the example of the hypergeometric differential equation on the three-punctured sphere in $[82,83]$, producing the corresponding four-dimensional Nekrasov partition function in the self-dual limit. On the physics side this programme has inspired a better understanding of the non-perturbative topological string partition function $[6,7,9]$.

\section{Acknowledgments}

This work is based on lectures given by the first author at the Pre-StringMath Summer School, 17-21 July 2017, and at StringMath 2017, 24-28 July 2017, Hamburg University.

LH wishes to thank Andrew Neitzke for years of discussions and collaboration on the topics of these notes, and the organisors of the Pre-StringMath Summer School and StringMath 2017 in Hamburg for the invitation to deliver these lectures, and for hospitality during the school and conference. We thank Andrew Neitzke for his Mathematica program swn-plotter [110]. The work of LH is supported by a Royal Society Dorothy Hodgkin Fellowship. The work of PR is supported by a James Watt Scholarship from Heriot-Watt University. The work of RJS was supported by the Consolidated Grant ST/P000363/1 from the Science and Technology Facilities Council.

\section{Spectral networks and abelianization}

In this section we introduce spectral networks and explain how each spectral network gives a coordinate system on the moduli space of flat connections. Spectral networks were first defined in [58], where it was shown that they are a fundamental ingredient in the story of BPS states and wall-crossing for $\mathcal{N}=2$ gauge theories of class $\mathcal{S}$ [54, 59]. In [58] it was also observed that spectral networks can be used to construct coordinate systems for moduli spaces of flat connections. This method, called abelianization, was further developed in [75]. In this section we follow the exposition of [75], while extending the abelianization method, as well as the notion of a Fenchel-Nielsen network, to Riemann surfaces with irregular punctures. $^{5}$

\subsection{WKB spectral networks}

A spectral network is a certain kind of network that can be drawn on a (possibly punctured) Riemann surface $C$. Suppose that $C$ has $n$ punctures at positions $z_{i}$ and write $C=$ $\bar{C} \backslash\left\{z_{1}, \ldots, z_{n}\right\}$, where $\bar{C}$ is compact. Our initial input is a fixed meromorphic quadratic differential $p_{2}$ on $C$, which can locally be written as

$$
p_{2}(z)=u(z) \mathrm{d} z \otimes \mathrm{d} z .
$$

\footnotetext{
${ }^{4}$ Very relevant here as well is the series of papers by Allegretti (see for instance [10, 11]) providing a mathematically rigorous framework for understanding the conformal limit of the spectral coordinates introduced by Gaiotto, Moore and Neitzke.

${ }^{5}$ We have used the Mathematica program swn-plotter from Andrew Neitzke [110] for many of the spectral network plots in these notes; we encourage the reader to play around with this her/himself.
} 
The differential $p_{2}$ may be singular at the punctures. A singular point is regular if $u(z)$ has a pole of order 2 there; otherwise it is irregular. In a neighbourhood of a regular puncture $z_{i},{ }^{6}$ the function $u(z)$ may be brought to the form

$$
u(z) \simeq \frac{m_{i}^{2}}{\left(z-z_{i}\right)^{2}},
$$

where $m_{i}$ are called the mass parameters. ${ }^{7}$

Consider the square root $\lambda=\sqrt{p_{2}}$. This is a one-form on $C$ which is locally given by $\lambda(z)=\sqrt{u(z)} \mathrm{d} z$. The one-form $\lambda$ is a priori multi-valued on $C$, but we will remedy this by choosing appropriate branch cuts on $C$. Fix a phase $\vartheta \in \mathbb{R} / 2 \pi \mathbb{Z}$. A $\vartheta$-trajectory is then a real curve $\gamma$ on $C$ such that

$$
\mathrm{e}^{-\mathrm{i} \vartheta} \sqrt{p_{2}}(v) \in \mathbb{R}^{\times}=\mathbb{R} \backslash\{0\}
$$

for any tangent vector $v$ to $\gamma$. This means that $\mathrm{e}^{-\mathrm{i} \vartheta} \sqrt{p_{2}}$ restricts to a real and non-vanishing one-form on $\gamma$. We can write this condition more concretely by choosing a parametrization $\gamma(t)$ of the curve and using the local form of $p_{2}$ as in (2.1). Then (2.3) says

$$
\mathrm{e}^{-\mathrm{i} \vartheta} \sqrt{u(\gamma(t))} \frac{\mathrm{d} \gamma(t)}{\mathrm{d} t} \in \mathbb{R}^{\times}
$$

The set of all $\vartheta$-trajectories form the leaves of a foliation of $C^{\prime}=C \backslash\left\{z \mid p_{2}(z)=0\right\}$. To see this, consider local coordinates $w(z)$ defined around any point $z_{0} \in C^{\prime}$ by

$$
w(z)=\int_{z_{0}}^{z} \sqrt{u\left(z^{\prime}\right)} \mathrm{d} z^{\prime} .
$$

Then $\sqrt{p_{2}(z)}=\mathrm{d} w$ and the $\vartheta$-trajectories are just straight line segments of inclination $\vartheta$. With respect to the coordinates $w$ we thus get a foliation whose leaves are straight lines of inclination $\vartheta .^{8}$

At a simple zero of $p_{2}$ the foliation by $\vartheta$-trajectories becomes singular. In a neighbourhood of the simple zero we can choose a local coordinate $z$ in which the zero is located at $z=0$; in this coordinate, locally $p_{2}(z) \simeq z \mathrm{~d} z \otimes \mathrm{d} z$. Consider a trajectory starting at $z=0$ as $\gamma(t)=\mathrm{e}^{\mathrm{i} \alpha} t$ for $t>0$. Then

$$
\sqrt{p_{2}}(v)=\sqrt{\gamma(t)} \frac{\mathrm{d} \gamma}{\mathrm{d} t}=\mathrm{e}^{\frac{3}{2} \mathrm{i} \alpha} t^{1 / 2} .
$$

The condition for $\gamma$ to be a $\vartheta$-trajectory is then

$$
\mathrm{e}^{-\mathrm{i} \vartheta} \mathrm{e}^{\frac{3}{2} \mathrm{i} \alpha} t^{1 / 2} \in \mathbb{R}^{\times}
$$

which yields

$$
\alpha=\frac{2 \vartheta}{3}+k \frac{2 \pi}{3} \quad \text { for } \quad k=0,1,2 .
$$

Thus there are three $\vartheta$-trajectories emitted from every simple zero of $p_{2}$ as shown in figure 1 .

On the other hand, around regular poles of $p_{2}$ the behaviour of $\vartheta$-trajectories depends on the value of the corresponding mass parameter $m$, as depicted in figure 2 .

\footnotetext{
${ }^{6}$ We use the terminology regular pole, regular singularity, and regular puncture interchangeably to mean the same thing.

${ }^{7}$ We will discuss irregular punctures later on and for the moment assume that $p_{2}$ has only regular singularities at all punctures.

${ }^{8}$ Note also that $\mathrm{d} w$ is non-vanishing on $C^{\prime}$ and thus defines a nowhere vanishing vector field on $C^{\prime}$.
} 


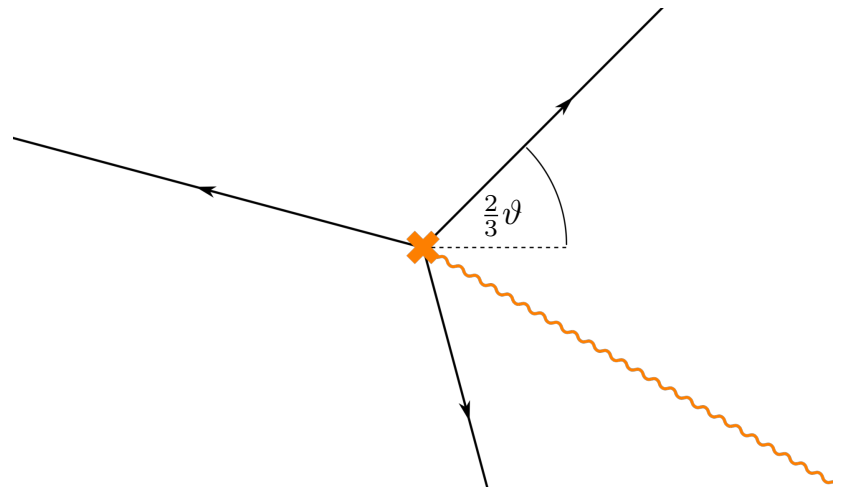

Figure 1. Illustration of the three $\vartheta$-trajectories emitted from a simple zero of $p_{2}$. The orange cross depicts the position of the zero, whereas the wavy line represents a choice of branch cut.

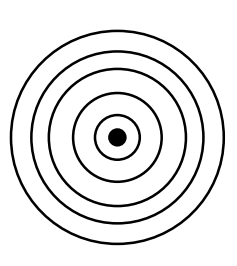

$m \mathrm{e}^{-\mathrm{i} \vartheta} \in \mathbb{R}$

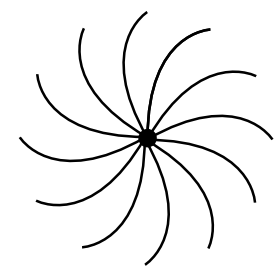

$\operatorname{Im}\left(m \mathrm{e}^{-\mathrm{i} \vartheta}\right)^{2}>0$

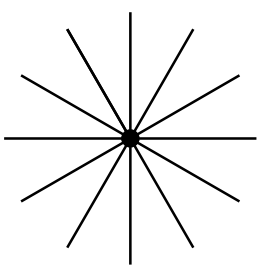

$m \mathrm{e}^{-\mathrm{i} \vartheta} \in \mathrm{i} \mathbb{R}$

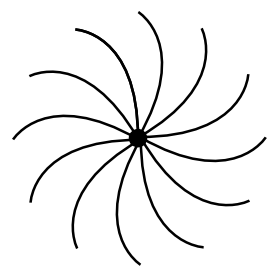

$\operatorname{Im}\left(m \mathrm{e}^{-\mathrm{i} \vartheta}\right)^{2}<0$

Figure 2. Behaviour of $\vartheta$-trajectories near a puncture (depicted as a solid circle) depending on the phase of $m \mathrm{e}^{-\mathrm{i} \vartheta}$.

A generic $\vartheta$-trajectory has both endpoints at punctures of $C$. We call a trajectory critical if one or both of its endpoints are zeroes of $p_{2}$. We then define the critical graph to be the union of all critical trajectories together with the zeroes of $p_{2}$. The trajectories may be oriented as outgoing from the zeroes, and, together with a choice of branch cuts of $\sqrt{p_{2}}$, labeled by either 12 or by 21 if the sign of $\sqrt{p_{2}}(v)$ is positive or negative, respectively. We call the critical graph together with orientations a WKB spectral network on $C$ and denote it by $\mathscr{W}\left(p_{2}, \vartheta\right)$.

Example 1. Consider $C=\mathbb{P}^{1} \backslash\{\infty\}=\mathbb{C}$ with the quadratic differential

$$
p_{2}(z)=\left(z^{2}+m\right) \mathrm{d} z \otimes \mathrm{d} z .
$$

This differential has an irregular singularity at $z=\infty$ of type $L=4$ (i.e. a pole of order six), since $p_{2}$ behaves as $z^{2} \mathrm{~d} z \otimes \mathrm{d} z$ near there. For $m>0$ and $\vartheta=(\pi / 2)^{+}$, we get the spectral network $\mathscr{W}$ shown in figure $3 \mathrm{~A}^{9}{ }^{9}$ Increasing the phase $\vartheta$ does not change the isotopy class of the network $\mathscr{W}$, but decreasing $\vartheta$ to $\vartheta_{\text {c }}=\pi / 2$ does. At $\vartheta=\vartheta_{\text {c }}$ the two trajectories in between the branch points overlap and form a saddle, as shown in figure $3 \mathrm{~B}$.

By decreasing $\vartheta$ further to $\vartheta=(\pi / 2)^{-}$we end up with the network in figure $3 \mathrm{C}$. That is, if we vary $\vartheta$ from $(\pi / 2)^{+}$to $(\pi / 2)^{-}$the isotopy class of $\mathscr{W}$ changes, with a saddle at the critical value $\vartheta_{\mathrm{c}}=\pi / 2$. We call such a topology change a flip. We will explain in section 3.3 why this physically describes the appearence of a BPS hypermultiplet of mass $m$.

\footnotetext{
${ }^{9}$ In figure 3 we have left out the branch cuts at infinity, which we rectify in example 7 below.
} 


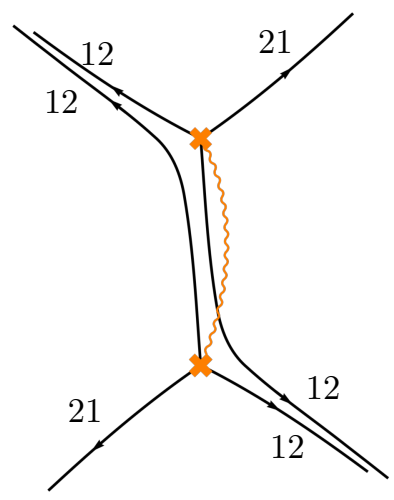

A

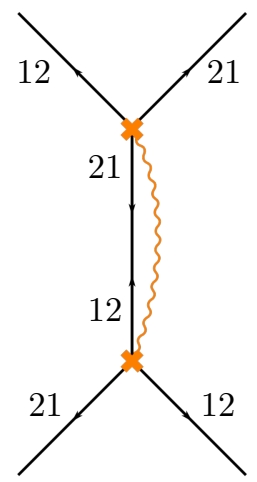

B

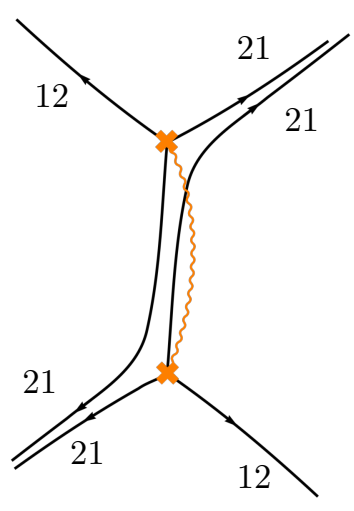

C

Figure 3. Spectral networks $\mathscr{W}\left(p_{2}, \vartheta\right)$ for $m>0$ as $\vartheta$ is varied.

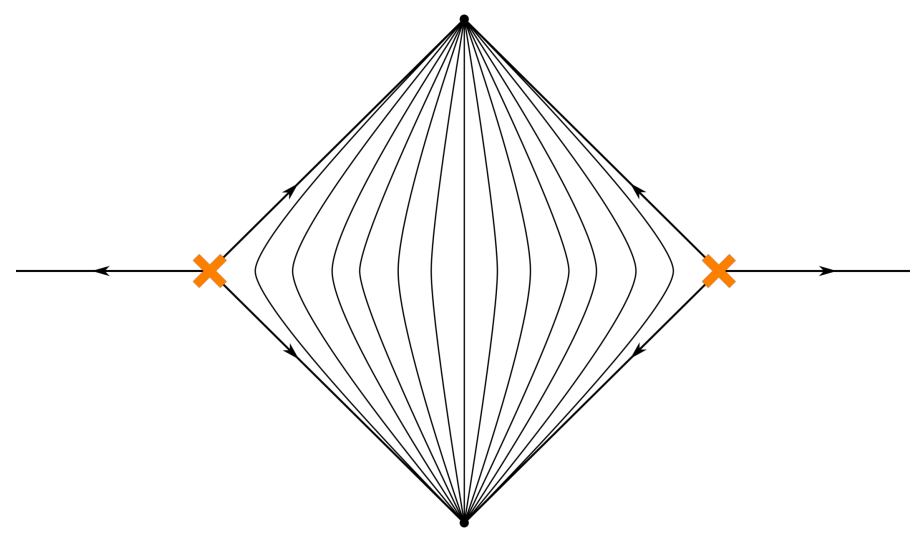

Figure 4. Topology of a cell of the foliation. The cell is bounded by critical trajectories running from a branch point to a puncture and it includes an infinite family of generic trajectories that run between the punctures.

Example 2. Let $C$ be any punctured Riemann surface, and take $p_{2}$ to be a generic differential with regular singularities at the punctures. For generic values of $\vartheta$ every trajectory ends on a puncture and the foliation defined by $p_{2}$ looks locally as in figure 4 . We call the resulting spectral network a Fock-Goncharov network (in the terminology of [75]). For a Fock-Goncharov network we can use the cells of the foliation to define an ideal triangulation $\mathscr{T}=\mathscr{T}\left(p_{2}, \vartheta\right)$ of $C$. The vertices of $\mathscr{T}$ are the punctures of $C$ and the edges are obtained by choosing one generic trajectory in each cell [59,75]. An example on the four-punctured sphere is shown in figure 5 .

Example 3. Consider $C=\mathbb{C}^{\times}=\mathbb{C} \backslash\{0\}$ with the quadratic differential

$$
p_{2}(z)=\left(\frac{1}{z^{3}}+\frac{9}{4 z^{2}}+\frac{1}{z}\right) \mathrm{d} z \otimes \mathrm{d} z .
$$




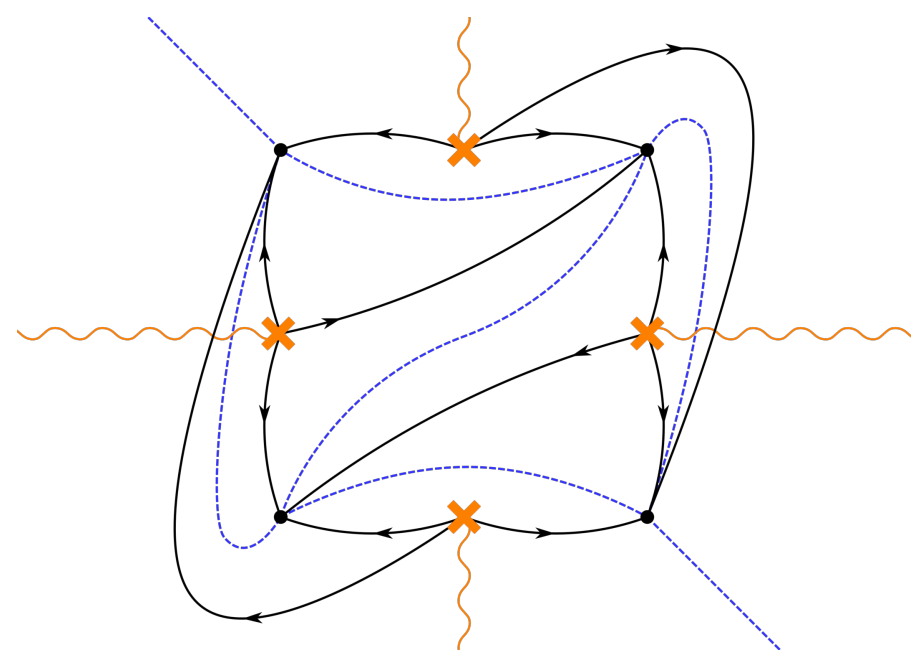

Figure 5. The four-punctured sphere (viewed as the plane with the point at infinity omitted) with a Fock-Goncharov network $\mathscr{W}$. The edges of an ideal triangulation $\mathscr{T}$ are indicated in blue.

This differential has irregular singularities at $z=0$ and $z=\infty$, since the behaviour of $p_{2}$ is given by $\frac{1}{\tilde{z}^{3}} \mathrm{~d} \tilde{z} \otimes \mathrm{d} \tilde{z}$ near there (in the local coordinate $\tilde{z}=z$ and $\tilde{z}=\frac{1}{z}$, respectively). Near $\vartheta=\vartheta_{\mathrm{c}}=\pi / 2$, something interesting happens: close to this critical phase, some trajectories start to wind around the cylinder $C=\mathbb{C}^{\times}$, see figure 6 . If one studies the winding for $\vartheta<\vartheta_{\mathrm{c}}$ (and the unwinding for $\vartheta>\vartheta_{\mathrm{c}}$ ), one finds that it is really an infinite sequence of flips; this is explained in detail in [59, section 5.9]. At $\vartheta=\vartheta_{c}$ the network changes topology. At this phase a family of closed trajectories emerges that surround the cylinder. This is illustrated in figure 6, and is called a juggle (see also [59, section 6.6.3]). As we will see in section 3.3, a juggle corresponds to a BPS vector multiplet in the corresponding supersymmetric field theory. The two limits $\vartheta \rightarrow \vartheta_{\mathrm{c}}^{ \pm}$are called the resolutions of the spectral network at the critical phase; they are illustrated schematically in figure 7 .

Example 4. If $C$ is any (possibly punctured) Riemann surface with regular punctures, we may consider a special class of quadratic differentials $\mathrm{e}^{-2 \mathrm{i} \vartheta} p_{2}$ for which all trajectories are compact and the critical graph is built out of saddles. These differentials are known as Strebel differentials. In particular, given any pants decomposition of $C$ along with a choice of "length" parameters $l_{k}$, there is a unique Strebel differential $\mathrm{e}^{-2 \mathrm{i} \vartheta} p_{2}$ respecting this pants decomposition and satisfying

$$
\mathrm{e}^{-\mathrm{i} \vartheta} \oint_{\alpha_{k}} \sqrt{p_{2}}=l_{k}
$$

for all pants curves $\alpha_{k}$. The corresponding spectral network is dual to the pants decomposition of $C$. We call such a network a Fenchel-Nielsen network (for more details see [75]). See figures 8 and 9 for examples of Fenchel-Nielson networks on the three-punctured and four-punctured sphere, respectively.

If the Riemann surface $C$ has irregular singularities, the Strebel condition is too strong (there are always non-compact trajectories in this case). Yet we would like to call a spectral 

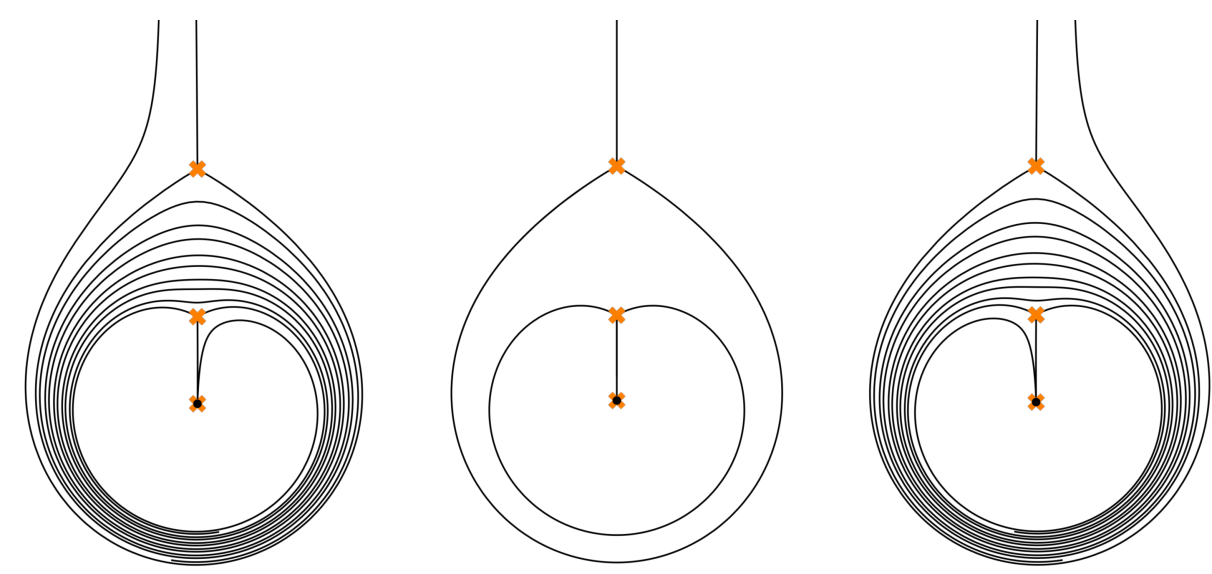

Figure 6. Spectral networks $\mathscr{W}\left(p_{2}, \vartheta\right)$, rotated through $-90^{\circ}$, for the quadratic differential (2.10) and phases $\vartheta_{\mathrm{c}}-\delta, \vartheta_{\mathrm{c}}, \vartheta_{\mathrm{c}}+\delta$, respectively, with $\vartheta_{\mathrm{c}}=\frac{\pi}{2}$ and $\delta$ small. The transition of the spectral network from $\vartheta_{\mathrm{c}}-\delta$ to $\vartheta_{\mathrm{c}}+\delta$ is called a juggle.
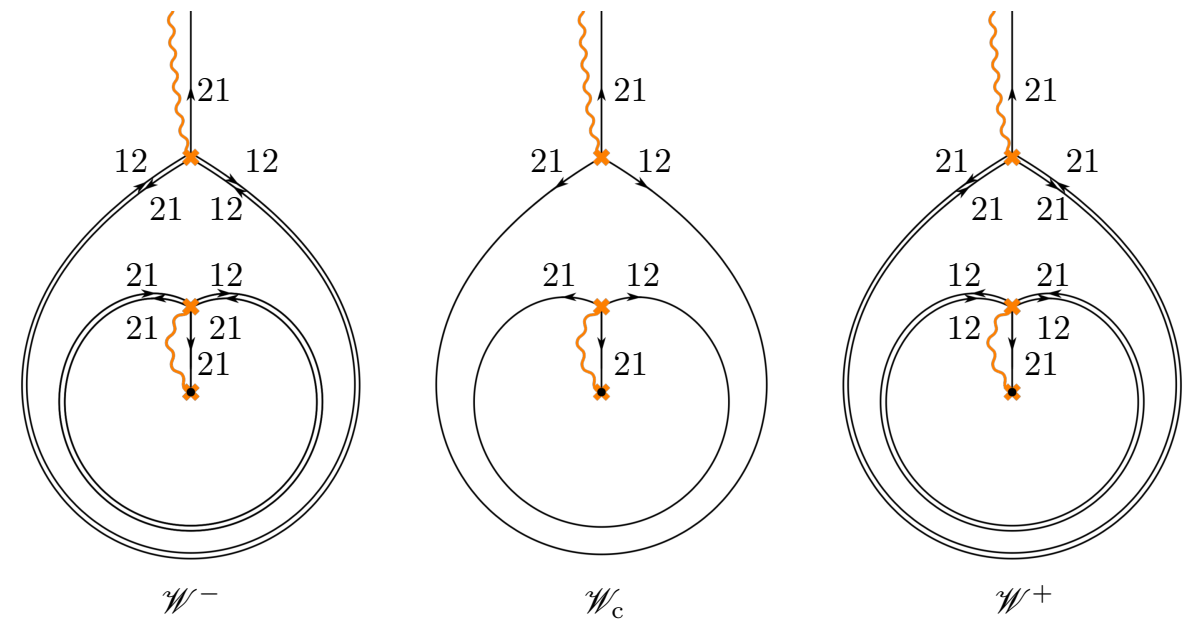

Figure 7. In the middle: the spectral network $\mathscr{W}_{\mathrm{c}}=\mathscr{W}\left(p_{2}, \vartheta_{\mathrm{c}}\right)$ for the quadratic differential (2.10) and critical phase $\vartheta_{\mathrm{c}}=\frac{\pi}{2}$. On either side: its resolutions $\mathscr{W}^{ \pm}=\mathscr{W}\left(p_{2}, \vartheta_{\mathrm{c}}^{ \pm}\right)$.

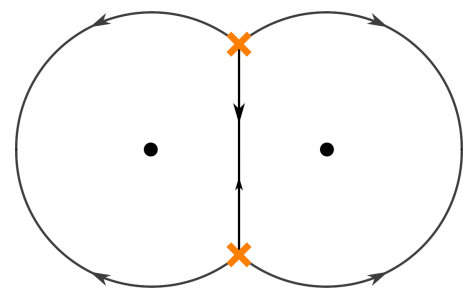

Figure 8. One of two Fenchel-Nielsen network topologies on the three-punctured sphere with the third puncture being a regular puncture at infinity. This is equivalent to a pair of pants, if the punctures are viewed as boundaries instead. 

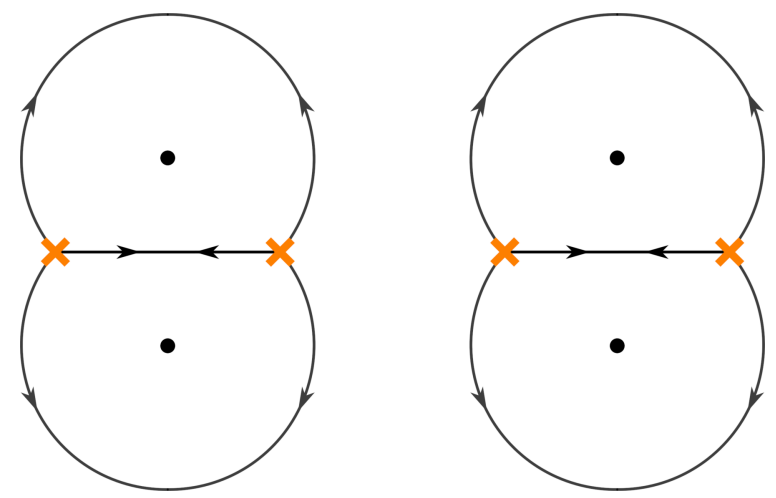

Figure 9. A Fenchel-Nielsen network on the four-punctured sphere glued together from two pairs of pants.

network $\mathscr{W}\left(p_{2}, \vartheta\right)$ of Fenchel-Nielsen type if there is a maximal number of non-degenerate ring domains (see also [35]). This is the case, for instance, at the critical phase $\vartheta_{\mathrm{c}}=\frac{\pi}{2}$ in example 3. We can thus consider the middle network in figure 6 as another example of a Fenchel-Nielsen network.

\subsection{Higher rank generalization}

So far we considered spectral networks defined by a single quadratic differential $p_{2}$. This may be generalized to a higher rank version of a WKB spectral network by considering a tuple of differentials $p=\left(p_{2}, \ldots, p_{K}\right)$ on $C$, where each $p_{k}$ is a meromorphic $k$-differential on $C$, possibly singular at the punctures $z_{i}$. In this case we call a singularity regular if the order of the pole of each $p_{k}$ is at most $k$, and irregular otherwise. As we will see later on, regular punctures may be classified by Young diagrams with $K$ boxes and at most $K-1$ rows.

The tuple of differentials $\left(p_{2}, \ldots, p_{K}\right)$ defines a (possibly branched) $K$-fold covering $\Sigma \subset T^{*} C$ of $C$, by the equation ${ }^{10}$

$$
\Sigma: \quad \lambda^{K}-\sum_{k=2}^{K} p_{k} \lambda^{K-k}=0,
$$

where $\lambda=w \mathrm{~d} z$ is the tautological one-form on the cotangent bundle $T^{*} C$. The curve $\Sigma$ is called the spectral curve, and we will encounter it again in section 3 when we explain how spectral networks are relevant to $\mathcal{N}=2$ field theories. In the following we assume that the covering $\Sigma \rightarrow C$ has only simple branch points, where exactly two sheets come together; this is the generalization of the requirement that $p_{2}$ has only simple zeroes for $K=2$.

Let $\lambda_{i}$ be the restriction of the tautological one-form $\lambda$ to the $i$-th sheet. We define an $i j$-trajectory on $C$ for $i \neq j$ to be a real curve $\gamma$ on $C$ such that

$$
\mathrm{e}^{-\mathrm{i} \vartheta}\left(\lambda_{i}-\lambda_{j}\right)(v) \in \mathbb{R}^{\times}
$$

\footnotetext{
${ }^{10}$ In $(2.12)$ we have changed the sign in front of the coefficients $p_{k}$ to match our conventions from section 2.1. This agrees with the conventions of [59], but unfortunately not with those of [58]. In particular, to verify the networks below, one needs to introduce an additional minus sign in swn-plotter [110].
} 


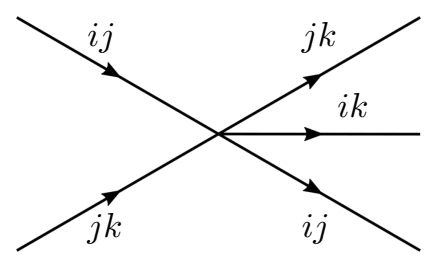

Figure 10. The crossing of walls with labels $i j$ and $j k$ results in a new wall with label $i k$ which is "born" at the intersection.

for any non-zero tangent vector $v$ to $\gamma$. We call such a trajectory critical if at least one of its endpoints is a branch point. The critical graph is again defined as the union of all critical trajectories, and we add an orientation and a label $i j$ to each $i j$-trajectory to get a spectral network $\mathscr{W}(p, \vartheta)$ on $C$. Sometimes we will call $\mathscr{W}(p, \vartheta)$ a spectral network subordinate to the cover $\Sigma \rightarrow C$.

There is a new phenomenon for critical trajectories in higher rank networks for which $K>2$ : trajectories with different labels (say $i j$ and $j k$ ) may cross paths and form a junction. For instance, the crossing of a 12 and a 23 trajectory will lead to a new trajectory of type 13, see figure 10. In this way new trajectories can be "born". The possibility of such crossings vastly increases the complexity of the higher rank networks. In fact, they are largely unexplored apart from the cases of higher rank generalizations of Fock-Goncharov and Fenchel-Nielsen networks [58, 75, 76].

Example 5. Let $K=3$ and $C=\mathbb{P}_{0,1, \infty}^{1}=\mathbb{P}^{1} \backslash\{0,1, \infty\}$ be the three-punctured sphere with the differentials

$$
\begin{aligned}
& p_{2}=\frac{c_{\infty} z^{2}-\left(c_{0}-c_{1}+c_{\infty}\right) z+c_{0}}{z^{2}(z-1)^{2}}(\mathrm{~d} z)^{\otimes 2} \\
& p_{3}=\frac{d_{\infty} z^{3}+u z^{2}+\left(d_{0}+d_{1}-d_{\infty}-u\right) z-d_{0}}{z^{3}(z-1)^{3}}(\mathrm{~d} z)^{\otimes 3}
\end{aligned}
$$

where $u \in \mathbb{C}$ is a free parameter, while

$$
c_{l}=-m_{l, 1}^{2}-m_{l, 1} m_{l, 2}-m_{l, 2}^{2} \quad \text { and } \quad d_{l}=m_{l, 1} m_{l, 2}\left(m_{l, 1}+m_{l, 2}\right),
$$

and $m_{l, 1} \neq m_{l, 2}$. The residues of these differentials at the regular punctures $z_{l}=l$ are given by $\left\{m_{l, 1}, m_{l, 2},-m_{l, 1}-m_{l, 2}\right\}$, respectively, and the spectral curve $\Sigma$ is a three-fold branched covering of $C$ with six simple branch points. This implies that $\Sigma$ is a punctured Riemann surface of genus one. In the limit $m_{l, j} \rightarrow 0$, the branch points of the covering $\Sigma \rightarrow C$ move towards the punctures and the topology of the corresponding spectral networks $\mathscr{W}(u, \vartheta)$ depends only on the phase of the quantity $\mathrm{e}^{-3 \mathrm{i} \vartheta} u$. For generic phase the network seems to be "wild", that is, it is dense in at least some parts of $C$. For non-generic phase the network is compact, with

$$
\mathrm{e}^{-\mathrm{i} \vartheta} \oint_{\gamma} \lambda \in \mathbb{R}
$$

for some one-cycle $\gamma$ on $\Sigma$. 


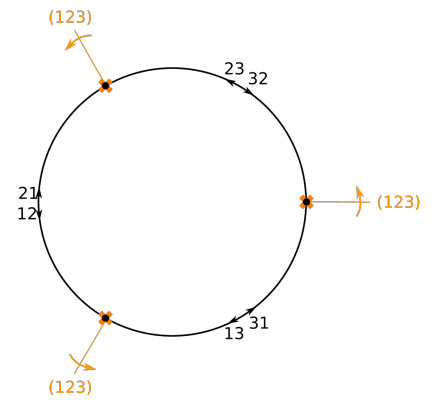

A

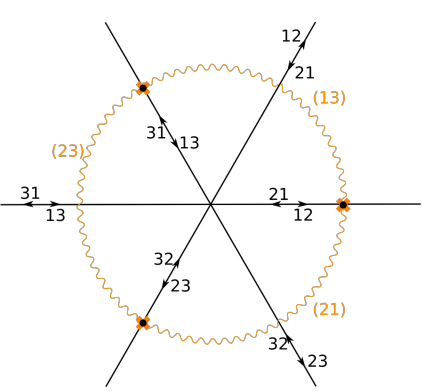

B

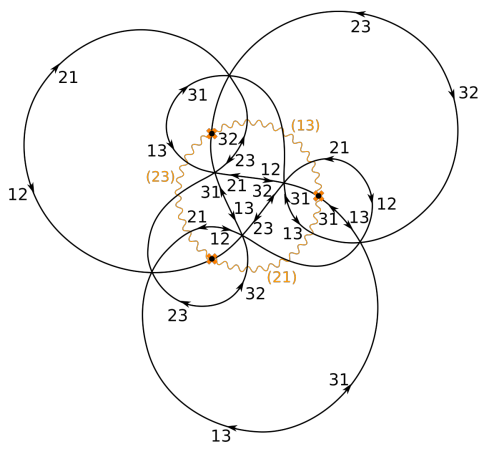

C

Figure 11. Three examples of higher rank Fenchel-Nielsen networks on the three-punctured sphere (taken from [76]): A $\mathscr{W}_{[1,0]} ; \mathbf{B} \quad \mathscr{W}_{[1,2]} ; \mathbf{C} \mathscr{W}_{[1,3]}$. Here we have moved the three punctures to $z=1, \omega, \omega^{2}$ with $\omega=\mathrm{e}^{2 \pi \mathrm{i} / 3}$.

These non-generic compact networks were studied in [76]. They are labelled by two coprime integers $p$ and $q$. The three simplest topologies $\mathscr{W}_{[1,0]}, \mathscr{W}_{[1,2]}$ and $\mathscr{W}_{[1,3]}$ are illustrated in figure 11. These networks are rather degenerate: if we were to perturb $\vartheta$ slightly away from the critical value, we would see that there is infinite winding around the saddles. For this reason we call these networks of higher rank Fenchel-Nielsen type.

As we will learn in section 3.3 , each network $\mathscr{W}_{[q, p]}$ encodes all BPS states of electromagnetic charge $(q, p)$ in the $E_{6}$ Minahan-Nemeschansky theory.

\subsection{Abelianization}

In the following we fix a branched $K$-fold cover $\pi: \Sigma \rightarrow C$ as in (2.12), and a spectral network $\mathscr{W}$ on $C$ subordinate to the covering. Let $\Sigma^{\prime}$ denote $\Sigma$ with the branch points removed. The general idea of abelianization is to lift non-abelian structures on a given rank $K$ vector bundle $E$ over $C$ to construct corresponding abelian structures on a line bundle $\mathcal{L}$ over the spectral cover $\Sigma^{\prime}$. In particular, starting from a flat $\mathrm{SL}(K, \mathbb{C})$ connection $\nabla$ on $C$, we construct a flat $\mathrm{GL}(1, \mathbb{C})$ connection $\nabla^{\mathrm{ab}}$ on $\Sigma^{\prime}$, using the data of the spectral network $\mathscr{W}$. This is illustrated in figure 12 . We first consider $\nabla$ on the connected components, or cells, of $C \backslash \mathscr{W}$. The cells are either contractible or tubular, and the construction works for either case [75]. On these we can find a gauge which diagonalizes $\nabla$. We then glue this patchwise diagonalization of $\nabla$ together by assigning unipotent gauge transformations to the trajectories of $\mathscr{W}$.

Concretely, in each cell we look for a basis of sections $\left(s_{1}, \ldots, s_{K}\right)$ of $E$ with respect to which $\nabla$ is diagonal,

$$
\nabla s_{i}=d_{i} \otimes s_{i},
$$

where $d_{i}$ are closed one-forms on $C$ for $i=1, \ldots, K$. On crossing a trajectory with label $i j$ we require a unipotent transformation

$$
s_{i} \longmapsto s_{i}+c_{i j} s_{j}=: s_{i}^{\prime} \quad \text { and } \quad s_{k} \longmapsto s_{k} \text { for } \quad k \neq i,
$$




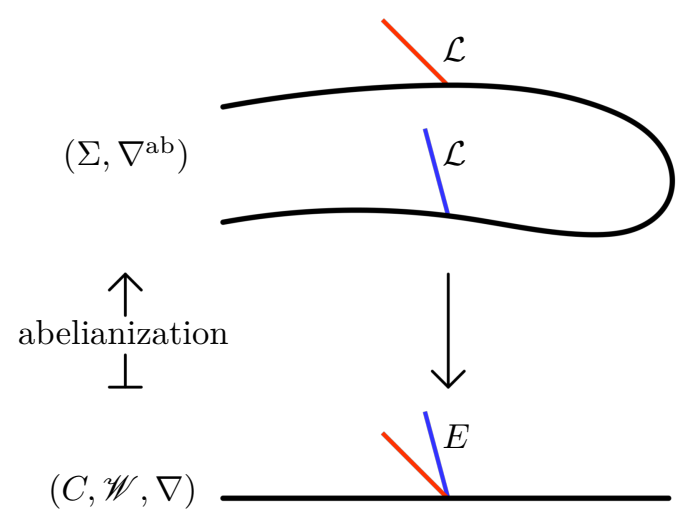

Figure 12. Given a spectral network $\mathscr{W}$ and a generic flat $\operatorname{SL}(K, \mathbb{C})$ connection $\nabla$ on the vector bundle $E$ over $C$, abelianization is a way of bringing $\nabla$ into an almost diagonal form, such that it may be lifted to a flat $\mathrm{GL}(1, \mathbb{C})$ connection $\nabla^{\mathrm{ab}}$ on the line bundle $\mathcal{L}$ over the spectral cover $\Sigma^{\prime}$.

for some function $c_{i j}$. On crossing a branch cut of type $(i j)$ we require that the sections on either side are related by a permutation matrix as

$$
s_{i} \longmapsto s_{j}, \quad s_{j} \longmapsto-s_{i} \quad \text { and } \quad s_{k} \longmapsto s_{k} \quad \text { for } \quad k \neq i, j .
$$

If we can find such a gauge, we may lift $\nabla$ on $C$ to a $\mathrm{GL}(1, \mathbb{C})$ connection $\nabla^{\text {ab }}$ on the spectral cover $\Sigma^{\prime}$ as follows. On $\Sigma \backslash \pi^{-1}(\mathscr{W})$ we define $\nabla^{\mathrm{ab}}$ on the $i$-th sheet by the diagonal entries $d_{i}$ of $\nabla$ :

$$
\nabla^{\mathrm{ab}}\left(s_{i}\right)=d_{i} \otimes s_{i} .
$$

To show that the unipotent gauge transformations (2.18) extend $\nabla^{\text {ab }}$ across the trajectories to all of $\Sigma$, we need to show that $\nabla s_{i}^{\prime}=d_{i} \otimes s_{i}^{\prime}$ for all $i=1, \ldots, K{ }^{11}$ Indeed, since $\nabla$ is an $\operatorname{SL}(K, \mathbb{C})$ connection, on crossing a trajectory with label $i j, \nabla s_{i}=d_{i} \otimes s_{i}$ is sent to

$$
\nabla s_{i}^{\prime}=d_{i}^{\prime} \otimes s_{i}^{\prime}=d_{i}^{\prime} \otimes s_{i}+d_{i}^{\prime} \otimes c_{i j} s_{j}
$$

with

$$
\nabla s_{i}^{\prime}=\nabla\left(s_{i}+c_{i j} s_{j}\right)=\nabla s_{i}+\mathrm{d} c_{i j} \otimes s_{j}+c_{i j} \nabla s_{j}=d_{i} \otimes s_{i}+\left(\mathrm{d} c_{i j}+c_{i j} d_{j}\right) \otimes s_{j},
$$

showing that $d_{i}^{\prime}=d_{i}$ as desired (and $\mathrm{d} c_{i j}=c_{i j}\left(d_{i}-d_{j}\right)$, so the functions $c_{i j}$ are bicovariantly constant). Note that, since $\nabla$ is an $\operatorname{SL}(K, \mathbb{C})$ connection, the connection $\nabla^{\text {ab }}$ carries some extra structure: parallel transport of the sections $\left(s_{1}, \ldots, s_{K}\right)$ along a path in $C \backslash \mathscr{W}$ (not crossing any branch cuts) is given by a diagonal matrix with determinant equal to 1 . We say that $\nabla^{\text {ab }}$ is equivariant, see [75, section 4.2] and [74, section 5.3].

To help us find such a gauge, and to show it is unique, we may need some additional discrete choices on $\nabla$; this is called a framing of $\nabla$.

Example 6. Fix a Fock-Goncharov network $\mathscr{W}$ and consider a flat $\mathrm{SL}(2, \mathbb{C})$ connection $\nabla$ on $C$. Locally $C \backslash \mathscr{W}$ contains cells of the form shown in figure $13 \mathrm{~A}$. For any puncture $z_{i}$,

\footnotetext{
${ }^{11}$ The connection $\nabla^{\mathrm{ab}}$ can be extended to a connection on all of $\Sigma$ which is almost flat: its holonomy around a simple branch point is $-\nVdash$.
} 


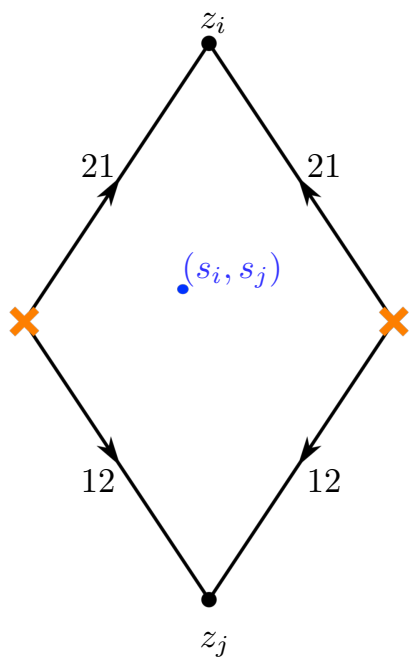

A

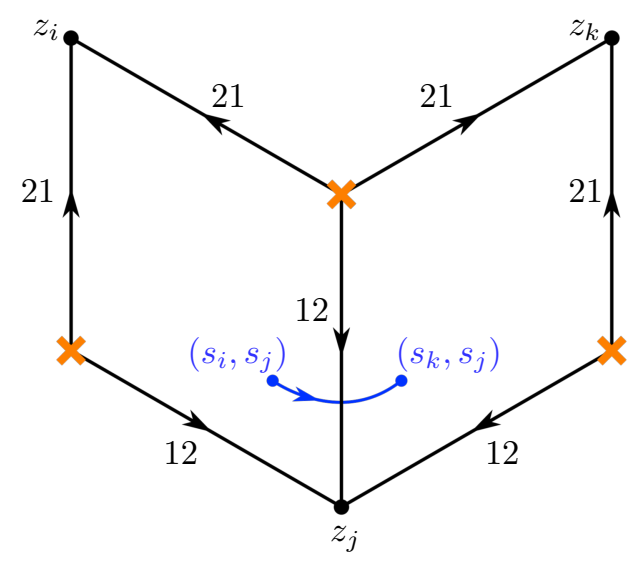

B

Figure 13. (A): a single cell of a Fock-Goncharov network, with basis of sections $\left(s_{i}, s_{j}\right)$. (B): adjacent cells with bases related by transport over a trajectory.

we defined the framing of $\nabla$ at $z_{i}$ to be a choice of a $\nabla$-invariant line sub-bundle $\ell_{i}$ of $E$ in a neighbourhood of $z_{i}$ with the following condition. If, for each cell with two punctures $z_{i}$ and $z_{j}$, we parallel transport the respective line bundles $\ell_{i}$ and $\ell_{j}$ to a common point $z$ in the cell, we require that $\ell_{i}(z) \neq \ell_{j}(z)$. We call the connection $\nabla$ together with this framing data a $\mathscr{W}$-framed connection.

Note that the condition above is automatically satisfied for a generic connection $\nabla$. Moreover, for generic $\nabla$ there are exactly $2^{n}$ possible $\mathscr{W}$-framings, where $n$ is the number of punctures. Indeed the monodromy around each puncture has two distinct eigenspaces, and a $\mathscr{W}$-framing corresponds to choosing just one of them.

How do we abelianize the $\mathscr{W}$-framed connection $\nabla$ ? Let us locally trivialize the cover $\Sigma$ over a cell with two punctures $z_{i}$ and $z_{j}$. Suppose that puncture $z_{i}$ has incoming trajectories of type 21 , whereas puncture $z_{j}$ has incoming trajectories of type 12 . The basis $\left(s_{i}, s_{j}\right)$ is then obtained by choosing $\left(s_{i} \in \ell_{i}, s_{j} \in \ell_{j}\right)$ for each cell.

The local picture for two neighbouring cells is shown in figure $13 \mathrm{~B}$. Each of the cells has basis $\left(s_{i}, s_{j}\right)$ defined as above, and crossing the trajectory between them gives a unipotent transformation that leaves one of the sections unchanged and modifies the other one. A choice of local bases of sections like this thus defines a $\mathscr{W}$-abelianization of $\nabla$. This can be shown to give a canonical one-to-one correspondence between $\mathscr{W}$-framings of $\nabla$ and $\mathscr{W}$-abelianizations of $\nabla[75$, section 5.2].

Example 7. Let us revisit example 1 with $C=\mathbb{C}$ and the spectral network $\mathscr{W}=\mathscr{W}\left(p_{2}, \vartheta_{c}\right)$ with

$$
p_{2}(z)=\left(z^{2}+m\right) \mathrm{d} z \otimes \mathrm{d} z \quad \text { and } \quad \vartheta_{\mathrm{c}}=\frac{\pi}{2} .
$$

Since $p_{2}$ has an irregular singularity at $z=\infty$ (a pole of order six), we consider flat $\operatorname{SL}(2, \mathbb{C}$ ) connections $\nabla$ with a corresponding irregular singularity at $z=\infty$. Any such connection 
$\nabla$ experiences the Stokes phenomenon at $z=\infty$. That is, it is impossible to find a single, well-defined section $s$ that is asymptotically small as $z \rightarrow \infty$. Instead, for this particular singularity, we require four sections $\tilde{s}_{1}, \tilde{s}_{2}, \tilde{s}_{3}$ and $\tilde{s}_{4}$ that each become asymptotically small as $z \rightarrow \infty$ along what is called a Stokes ray. (In section 4.4 we will write down explicit expressions for the sections $\tilde{s}_{i}$ when $\nabla$ is an oper connection.)

To keep track of the angular information, we consider the blow-up of the singularity at infinity and replace the puncture at $z=\infty$ with a small circle $S^{1}$ bounding an infinitesimal disc $D_{\infty}$. We then mark four points $\tilde{z}_{i}$ on this circle corresponding to the Stokes rays, and to these points we assign the sections $\tilde{s}_{i}$ that become asymptotically small along the corresponding rays. Each section $\tilde{s}_{i}$ has good asymptotics in the angular regions adjacent to the Stokes ray labeled by $\tilde{s}_{i}$. On the overlap of the regions where two sections are well-defined, they are related by a Stokes matrix [22].

To frame the connection $\nabla$ at infinity, we similarly replace the puncture at $z=\infty$ by a small circle $S^{1}$ with four marked points $z_{i}$ corresponding to the four incoming trajectories. The framing of $\nabla$ then corresponds to a choice of $\nabla$-invariant sub-bundle $\ell_{i}$ for every marked point $z_{i}$, again with the constraint that if two marked points $z_{i}$ and $z_{j}$ are connected by a path which does not cross any trajectories, and we parallel transport $\ell_{i}$ and $\ell_{j}$ to a common point $z$ on this path, then $\ell_{i}(z) \neq \ell_{j}(z)$. More precisely, consider the network $\mathscr{W}\left(p_{2}, \vartheta\right)$ for either $\vartheta=(\pi / 2)^{+}$or $\vartheta=(\pi / 2)^{-}$; these are sometimes called the two resolutions of the (Fenchel-Nielsen type) network $\mathscr{W}$, and we denote them by $\mathscr{W}^{ \pm}$respectively. ${ }^{12}$ Then the choice of framing $\ell_{1}, \ell_{2}, \ell_{3}, \ell_{4}$ determines a unique $\mathscr{W}^{ \pm}$-abelianization of $\nabla$, whose basis of sections in each cell is shown in figure 14, with $s_{i} \in \ell_{i}$. Note that the two bases on either side of a trajectory are indeed related by a unipotent transformation of the type (2.18). On either side of a branch cut they are related by a permutation matrix as in (2.19). (To trivialize the covering $\Sigma \rightarrow C$, we choose not only the branch cuts connecting the two branch points, but also an additional two branch cuts at $z=\infty$.) The bases of sections for each network $\mathscr{W}^{ \pm}$, as illustrated in figure 14, brings the connection $\nabla$ in an almost-diagonal gauge, so that it may be lifted to a $\mathrm{GL}(1, \mathbb{C})$ connection $\nabla_{ \pm}^{\mathrm{ab}}$ on the spectral cover $\Sigma$ by identifying the two elements $\left(s_{i}, s_{j}\right)$ of the basis in each cell with the sheets of $\Sigma$.

Example 8. Let us now go back to example 3, where $C=\mathbb{C}^{\times}$and the spectral network $\mathscr{W}\left(p_{2}, \vartheta_{\mathrm{c}}\right)$ is defined by (see figures 6 and 7 )

$$
p_{2}(z)=\left(\frac{1}{z^{3}}+\frac{9}{4 z^{2}}+\frac{1}{z}\right) \mathrm{d} z \otimes \mathrm{d} z \quad \text { and } \quad \vartheta_{\mathrm{c}}=\frac{\pi}{2} .
$$

Since $p_{2}$ has irregular singularities at $z=0$ and $z=\infty$, we consider flat $\operatorname{SL}(2, \mathbb{C})$ connections $\nabla$ with a corresponding irregular singularity at these points. Any such connection $\nabla$ may have a monodromy around the cylinder and is described by Stokes theory at $z=0$ and $z=\infty$. For this particular type of singularity (the mildest case where $p_{2}$ has a pole of order three), there is just one Stokes ray emitted from each puncture.

\footnotetext{
${ }^{12}$ It is not possible to abelianize precisely at the critical phase $\vartheta_{c}=\pi / 2$, we have to make a choice of resolution.
} 

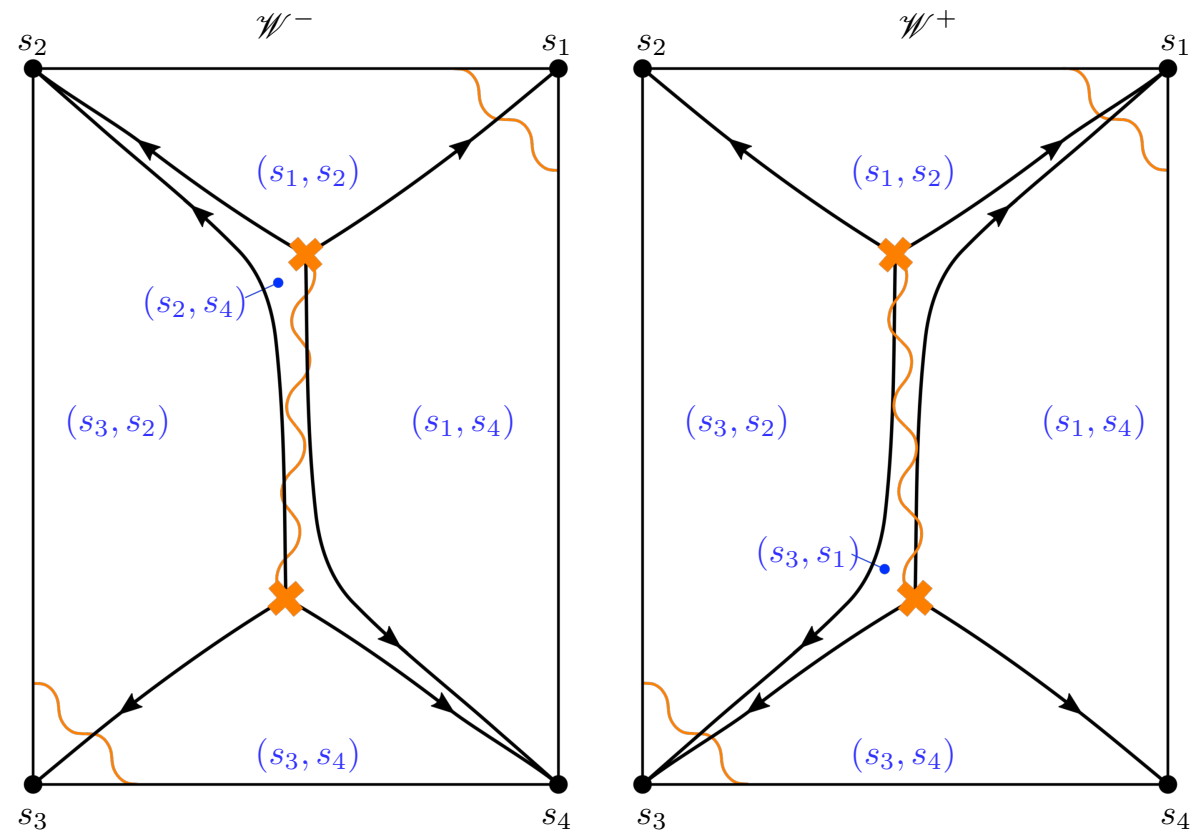

Figure 14. Bases of sections $\left(s_{i}, s_{j}\right)$ in the cells of $C \backslash \mathscr{W}^{ \pm}$defining the abelianization of $\nabla$ for the two networks $\mathscr{W}^{ \pm}$.

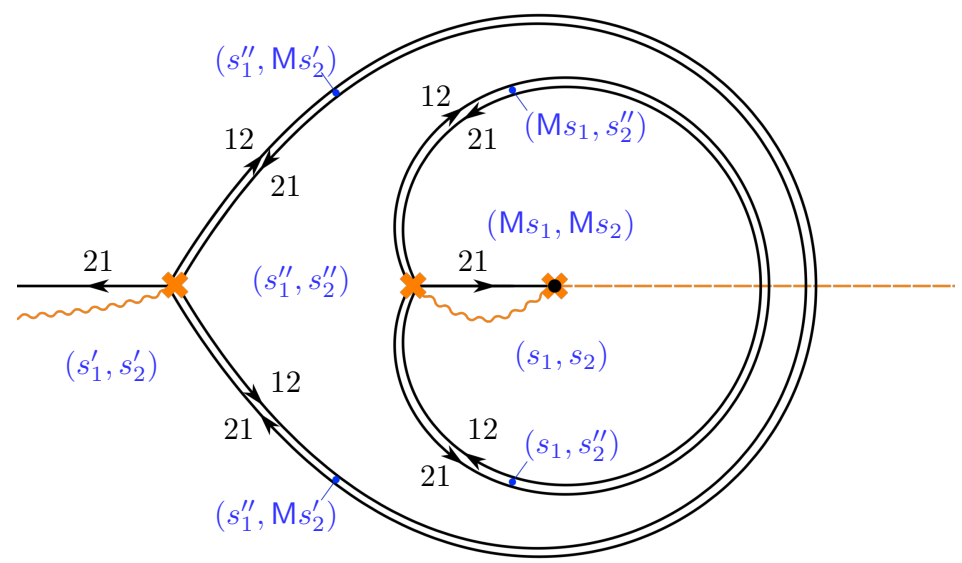

Figure 15. Bases of sections for the resolution $\mathscr{W}^{-}$. The dashed orange line is a monodromy cut for the monodromy around the cylinder. Note that $\mathrm{M} s_{1}=s_{2}$ and $\mathrm{M} s_{1}^{\prime}=s_{2}^{\prime}$.

To frame the connection $\nabla$ we choose $\nabla$-invariant line bundles $\ell$ and $\ell^{\prime}$ in the neighbourhood of $z=0$ and $z=\infty$, respectively, as well as an eigenline $\ell^{\prime \prime}$ of the counterclockwise monodromy $\mathrm{M}$ of $\nabla$ around $z=0$. With this choice of framing data there is a unique $\mathscr{W}^{ \pm}$ abelianization of $\nabla$, for each of the two resolutions $\mathscr{W}^{ \pm}$of $\mathscr{W}\left(p_{2}, \vartheta_{\mathrm{c}}\right)$. The abelianization of $\nabla$ with respect to the resolution $\mathscr{W}^{-}$is illustrated in figure 15 .

This abelianization is characterized by the bases of sections shown in figure 15 (in agreement with [77, section 4.3]). In this figure $s_{i}$ and $s_{i}^{\prime}$ are local bases of sections in the neighbourhood of the incoming trajectories at $z=0$ and $z=\infty$, respectively, with $s_{1} \in \ell$ 
and $s_{1}^{\prime} \in \ell^{\prime}$, and $s_{i}^{\prime \prime}$ are local sections in some simply-connected domain of the intermediate annulus, with $s_{2}^{\prime \prime} \in \ell^{\prime \prime}$ (in the conventions of [75, sections 5.2 and 5.3]).

After possibly rescaling some of these sections, the change of basis matrices can be brought in the desired triangular form. For instance, the matrix taking $\left(s_{1}, s_{2}\right)$ to $\left(s_{1}^{\prime \prime}, s_{2}^{\prime \prime}\right)$ has the form

$$
\left(\begin{array}{ll}
* & * \\
0 & *
\end{array}\right)\left(\begin{array}{ll}
* & 0 \\
* & *
\end{array}\right)
$$

relative to the basis $\left(s_{1}, s_{2}\right)$ in resolution $\mathscr{W}^{+}$, and

$$
\left(\begin{array}{ll}
* & 0 \\
* & *
\end{array}\right)\left(\begin{array}{ll}
* & * \\
0 & *
\end{array}\right)
$$

in resolution $\mathscr{W}^{-}$.

It is not always that easy (or even possible) to find a suitable framing on $\nabla$ which makes the abelianization process one-to-one. A nice example of this is the circular higher Fenchel-Nielsen network of example 5. Here it turns out that there are generically 12 abelianizations which may be identified with the singular fibers of an auxiliary rational elliptic surface [77, section 6].

\subsection{Spectral coordinates}

One of the applications of $\mathscr{W}$-abelianization is the construction of a system of Darboux coordinates on the moduli space $\mathscr{M}_{\text {flat }}^{\mathscr{W}}(C, \mathrm{SL}(K, \mathbb{C}))$ of $\mathscr{W}$-framed flat $\mathrm{SL}(K, \mathbb{C})$ connections on $C$. This moduli space is of importance for example in the study of the Hitchin system, and it also shows up in a variety of other problems in mathematical physics.

Given a basis of one-cycles on $\Sigma$, we first define a coordinate system on the moduli space $\mathscr{M}_{\text {flat }}^{\prime}(\Sigma, \mathrm{GL}(1, \mathbb{C}))$ of equivariant $\mathrm{GL}(1, \mathbb{C})$ connections on $\Sigma$ via the holonomies

$$
\mathcal{X}_{\gamma}=\operatorname{Hol}_{\gamma} \nabla^{\mathrm{ab}} \in \mathbb{C}^{\times},
$$

which depend only on the homology class $[\gamma] \in \Gamma=H_{1}(\bar{\Sigma}, \mathbb{Z})$ of a one-cycle $\gamma$. Through $\mathscr{W}$-abelianization they also give a system of coordinates $\left\{\mathcal{X}_{\gamma}^{\mathscr{W}}\right\}_{\gamma \in \Gamma}$ on $\mathscr{M}_{\text {flat }}^{\mathscr{W}}(C, \mathrm{SL}(K, \mathbb{C}))$, by first abelianizing $\nabla$ into $\nabla_{\mathscr{W}}^{\mathrm{ab}}$ and then using (2.27). We refer to these coordinates as spectral coordinates, since they are defined in terms of spectral data, i.e. data on the spectral curve $\Sigma$. Note that this coordinate system depends only on the isotopy class of the network $\mathscr{W}$.

The moduli space $\mathscr{M}_{\text {flat }}^{\mathscr{W}}(C, \mathrm{SL}(K, \mathbb{C}))$ has a natural holomorphic symplectic form given locally by the Atiyah-Bott-Goldman formula [15, 63]

$$
\Omega=\frac{1}{2} \int_{C} \operatorname{Tr}(\delta \mathcal{A} \wedge \delta \mathcal{A}),
$$

where $\mathcal{A}$ is the connection one-form of a $\mathscr{W}$-framed flat $\operatorname{SL}(K, \mathbb{C})$ connection $\nabla$ on $C$. Any spectral coordinate system $\left\{\mathcal{X}_{\gamma}\right\}_{\gamma \in \Gamma}$ consists of (exponentiated) holomorphic Darboux 


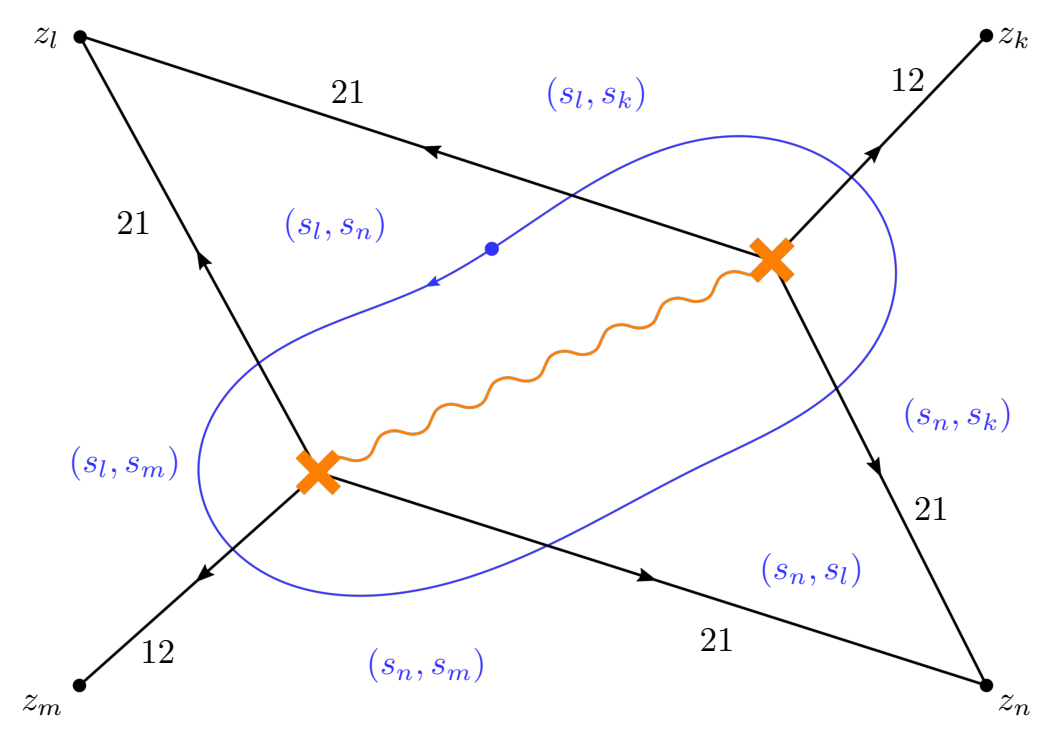

Figure 16. A local Fock-Goncharov network with one-cycle $\gamma$ indicated in blue, and the data needed to compute its holonomy.

coordinates with respect to this holomorphic symplectic form, as it brings the corresponding holomorphic Poisson structure $\{\cdot, \cdot\}$ on $\mathscr{M}_{\text {flat }}^{\mathscr{W}}(C, \mathrm{SL}(K, \mathbb{C}))$ to the form

$$
\left\{\mathcal{X}_{\gamma}, \mathcal{X}_{\gamma^{\prime}}\right\}=\left\langle\gamma, \gamma^{\prime}\right\rangle \mathcal{X}_{\gamma} \mathcal{X}_{\gamma^{\prime}}=\left\langle\gamma, \gamma^{\prime}\right\rangle \mathcal{X}_{\gamma+\gamma^{\prime}}
$$

where $\langle\cdot, \cdot\rangle$ is the intersection pairing on $\Gamma$. In examples, we may compute the coordinates $\mathcal{X}_{\gamma}$ explicitly through abelianization in terms of framing data [58].

Example 9. Suppose $K=2$, and consider a Fock-Goncharov network $\mathscr{W}$. Given a $\mathscr{W}$ framed $\operatorname{SL}(2, \mathbb{C})$ connection $\nabla$ and a one-cycle $\gamma$ on $\Sigma$, we would like to compute the holonomy $\mathcal{X}_{\gamma}$ of the corresponding $\mathrm{GL}(1, \mathbb{C})$ connection $\nabla^{\mathrm{ab}}$ along $\gamma$. The local geometry of a generic one-cycle $\gamma$ in a Fock-Goncharov network is illustrated in figure 16.

Before starting the computation, let us recall that crossing a trajectory of type 12 maps the basis from $\left(s_{i}, s_{j}\right)$ to

$$
s_{j} \longmapsto s_{j} \quad \text { and } \quad s_{i} \longmapsto s_{i}+c_{i j} s_{j}
$$

Suppose that the basis of sections on the other side of the trajectory is $\left(s_{k}, s_{j}\right)$. Then $s_{i}^{\prime}=s_{i}+c_{i j} s_{j}$ must be proportional to the already chosen basis section $s_{k}$ :

$$
s_{i}^{\prime}=\lambda s_{k} .
$$

Taking the exterior product on both sides with $s_{j}$, we find

$$
\lambda=\frac{s_{i} \wedge s_{j}}{s_{k} \wedge s_{j}}
$$


We may thus alternatively write the mapping of $s_{i}$ as

$$
s_{i} \longmapsto s_{i}^{\prime}=\frac{s_{i} \wedge s_{j}}{s_{k} \wedge s_{j}} s_{k}
$$

In a similar manner, a trajectory of type 21 leaves the first section in the basis $\left(s_{i}, s_{j}\right)$ unchanged and maps the second section according to (2.33). It is useful to note that the trajectory going into a puncture $z_{i}$ leaves invariant the section $s_{i}$ associated to it.

Let us now begin the computation of the holonomy of $\nabla^{\mathrm{ab}}$ along $\gamma$. Start at the marked point on the first sheet with section $s_{l}$. Note that parallel transport by $\nabla^{\mathrm{ab}}$ along $\gamma$ will multiply the section $s_{l}$ by this holonomy:

$$
s_{l} \longmapsto \mathcal{X}_{\gamma} s_{l}
$$

We can compute $\mathcal{X}_{\gamma}$ by following the path $\gamma$ and using the rules (2.33) when crossing a trajectory. The first trajectory we cross goes into $z_{l}$ and thus does not change $s_{l}$. We then cross the trajectory going into $z_{m}$ and find

$$
s_{l} \longmapsto \frac{s_{l} \wedge s_{m}}{s_{n} \wedge s_{m}} s_{n}
$$

This is left unchanged as we cross the third and fourth trajectories, as both of these go into $z_{n}$. The next trajectory goes into $z_{k}$ and implies

$$
s_{n} \longmapsto \frac{s_{n} \wedge s_{k}}{s_{l} \wedge s_{k}} s_{l},
$$

which is again left invariant when we cross the last trajectory and close the cycle. Hence in the end, by following the path $\gamma$ we arrive at the section $s_{l}$ multiplied by the cross-ratio

$$
\mathcal{X}_{\gamma}=\frac{s_{n} \wedge s_{k}}{s_{l} \wedge s_{k}} \frac{s_{l} \wedge s_{m}}{s_{n} \wedge s_{m}}
$$

This is the spectral coordinate that we set out to compute. It is indeed the familiar formula for a Fock-Goncharov coordinate on the moduli space of framed flat $\mathrm{SL}(2, \mathbb{C})$-connections on $C$, attached to the ideal triangulation $\mathscr{T}$ of $C$ dual to the Fock-Goncharov network $\mathscr{W}[59,60]$. These coordinates and their generalizations to higher rank are for instance useful for studying the quantization of the moduli spaces $\mathscr{M}_{\text {flat }}^{\mathscr{W}}(C, \mathrm{SL}(K, \mathbb{C}))$.

Example 10. Going back to example 7, we first need to address a subtlety. Flat $\operatorname{SL}(2, \mathbb{C})$ connections on this geometry may experience a monodromy around the irregular singularity at infinity, which is usually fixed. This implies that the moduli space of such connections is actually zero-dimensional. However, we may consider a larger moduli space (of complex dimension two) by considering flat $\mathrm{SL}(2, \mathbb{C})$ connections with any monodromy around infinity, and where we instead fix the gauge at infinity. This latter requirement means choosing a trivialization of the bundle $E$ at infinity.

This extended moduli space can be parameterized by two spectral coordinates $\mathcal{X}_{A}$ and $\mathcal{X}_{B}$ corresponding to the two one-cycles shown in figure 17 . Note that the $B$-cycle begins and ends on a different sheet of the spectral covering $\Sigma$, and $\mathcal{X}_{B}$ is only a holonomy 

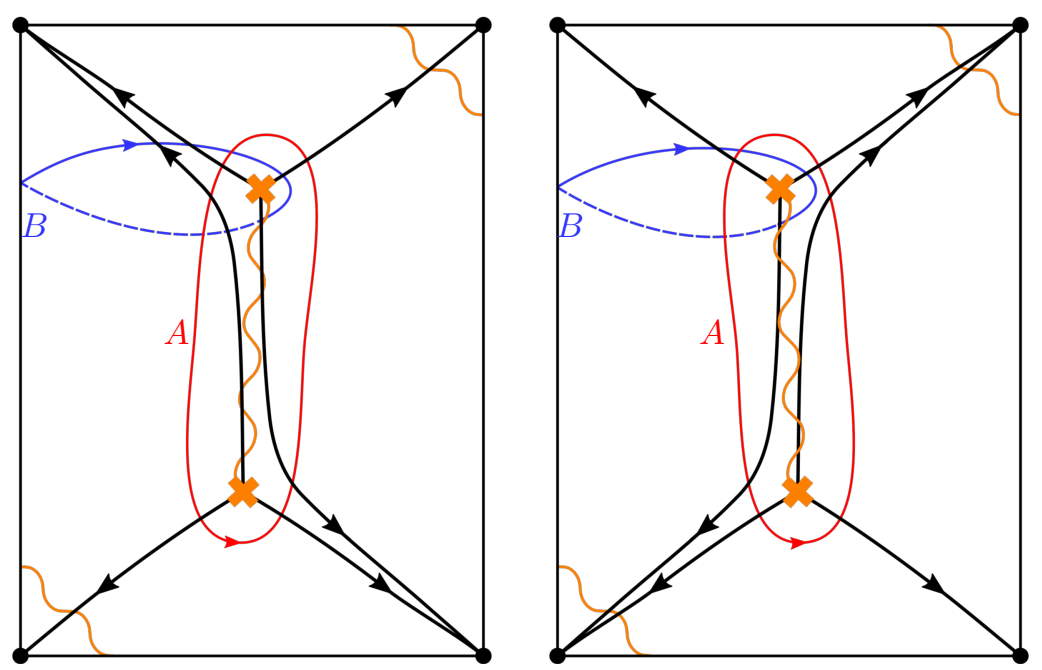

Figure 17. Choice of $A$-cycle and $B$-cycle on $\Sigma$ for $\mathscr{W}^{ \pm}$. The $B$-cycle is non-compact and requires the gauge at infinity to be fixed.

invariant because we fixed the gauge at infinity. The coordinates $\mathcal{X}_{A}$ and $\mathcal{X}_{B}$ can be computed similarly to example 9 . We find that $\mathcal{X}_{A}$ is given by the cross-ratio

$$
\mathcal{X}_{A}=\frac{s_{1} \wedge s_{2}}{s_{3} \wedge s_{2}} \frac{s_{3} \wedge s_{4}}{s_{1} \wedge s_{4}}
$$

for both resolutions $\mathscr{W}^{+}$and $\mathscr{W}^{-}$, while $\mathcal{X}_{B}$ can be brought in the form

$$
\mathcal{X}_{B}^{+}=\frac{s_{1} \wedge s_{4}}{s_{2} \wedge s_{4}} \quad \text { and } \quad \mathcal{X}_{B}^{-}=-\frac{s_{1} \wedge s_{3}}{s_{1} \wedge s_{2}}
$$

for the networks $\mathscr{W}^{+}$and $\mathscr{W}^{-}$, respectively. The gauge fixing at infinity means that the sections $s_{3}$ and $s_{4}$ are completely fixed (a GL $(1, \mathbb{C})$ gauge transformation would multiply them by a constant), so that $\mathcal{X}_{B}^{ \pm}$are indeed invariants. It may seem like there are various other choices to be made for the $B$-cycle, but it actually turns out that all of them result in a spectral coordinate that is equal to $\mathcal{X}_{B}$ up to multiplication by a power of $\mathcal{X}_{A}$. This follows easily after realizing that the exterior products $s_{1} \wedge s_{2}, s_{1} \wedge s_{4}, s_{3} \wedge s_{4}$ and $s_{3} \wedge s_{2}$ are equal to one another, up to the monodromy $\mathcal{X}_{A}$. This is because $\nabla$ is an $\operatorname{SL}(2, \mathbb{C})$ connection and hence the exterior products $s_{i} \wedge s_{j}$ are invariant after crossing a trajectory. ${ }^{13}$

Example 11. Consider the Fenchel-Nielsen network from example 8, with the choice of $A$-cycle and $B$-cycle on $\Sigma$ as shown in figure 18. The spectral coordinate $\mathcal{X}_{A}$ is simply an eigenvalue of the monodromy matrix $\mathrm{M}$ of $\nabla$, since the one-cycle $A$ on $\Sigma$ does not cross any trajectories. (More precisely, it is the eigenvalue corresponding to the eigenvector $s_{2}^{\prime \prime}$.) The coordinate $\mathcal{X}_{B}$, on the other hand, is given by the cross-ratio

$$
\mathcal{X}_{B}^{-}=\mathcal{X}_{A}^{2} \frac{\left(s_{1} \wedge s_{2}^{\prime \prime}\right)^{2}\left(s_{1}^{\prime} \wedge s_{1}^{\prime \prime}\right)^{2}}{\left(s_{1} \wedge s_{2}\right)\left(s_{1}^{\prime} \wedge s_{2}^{\prime}\right)\left(s_{1}^{\prime \prime} \wedge s_{2}^{\prime \prime}\right)^{2}}
$$

\footnotetext{
${ }^{13}$ One should exercise caution here, since these relations only hold locally and do not account for possible monodromies in non-simply connected regions.
} 


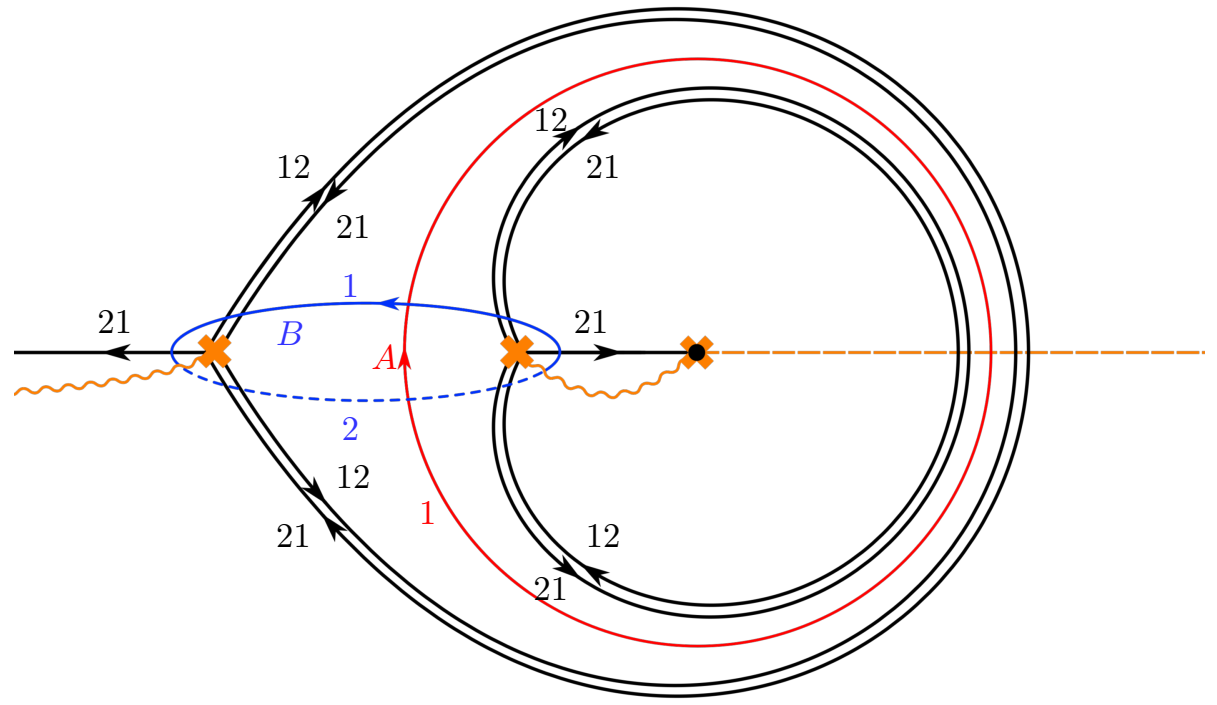

Figure 18. Choice of $A$-cycle and $B$-cycle for $\mathscr{W}^{-}$.

in resolution $\mathscr{W}^{-}$, and by

$$
\mathcal{X}_{B}^{+}=\mathcal{X}_{A}^{-2} \frac{\left(s_{1} \wedge s_{2}\right)\left(s_{1}^{\prime} \wedge s_{2}^{\prime}\right)\left(s_{1}^{\prime \prime} \wedge s_{2}^{\prime \prime}\right)^{2}}{\left(s_{1} \wedge s_{1}^{\prime \prime}\right)^{2}\left(s_{1}^{\prime} \wedge s_{2}^{\prime \prime}\right)^{2}}
$$

in resolution $\mathscr{W}^{+}$, where the sections $s_{i}, s_{i}^{\prime}$ and $s_{i}^{\prime \prime}$ are all defined in example 8 (see figure 15 ). Similarly to the previous examples, this follows by computing the parallel transport of $\nabla^{\text {ab }}$ along the one-cycle $B$ on $\Sigma$ and using the rules (2.33) when crossing a trajectory.

To compute $\mathcal{X}_{B}^{-}$, consider the setup in figure 15 , where we have introduced a mondromy cut (the dashed orange line) to take care of the monodromy that the local sections $s_{i}$, $s_{i}^{\prime}$ and $s_{i}^{\prime \prime}$ experience under parallel transport around the $A$-cycle. Using the identities $\mathrm{M} s_{1}=s_{2}, \mathrm{M} s_{1}^{\prime}=s_{2}^{\prime}, \mathrm{M} s_{1}^{\prime \prime}=\mu^{-1} s_{1}^{\prime \prime}$ and $\mathrm{M} s_{2}^{\prime \prime}=\mu s_{2}^{\prime \prime}$ with $\mu=\mathcal{X}_{A}$, as well as the fact that $\mathrm{M} s \wedge \mathrm{M} s^{\prime}=s \wedge s^{\prime}$, we indeed find

$$
\mathcal{X}_{B}^{-}=\frac{s_{2}^{\prime} \wedge s_{1}^{\prime \prime}}{s_{2}^{\prime \prime} \wedge s_{1}^{\prime \prime}} \frac{s_{2}^{\prime \prime} \wedge s_{1}}{s_{2} \wedge s_{1}} \frac{s_{2} \wedge s_{2}^{\prime \prime}}{s_{1}^{\prime \prime} \wedge s_{2}^{\prime \prime}} \frac{s_{1}^{\prime \prime} \wedge \mathrm{M} s_{2}^{\prime}}{s_{2}^{\prime} \wedge \mathrm{M} s_{2}^{\prime}}=\mu^{2} \frac{\left(s_{1} \wedge s_{2}^{\prime \prime}\right)^{2}\left(s_{1}^{\prime} \wedge s_{1}^{\prime \prime}\right)^{2}}{\left(s_{1} \wedge s_{2}\right)\left(s_{1}^{\prime} \wedge s_{2}^{\prime}\right)\left(s_{1}^{\prime \prime} \wedge s_{2}^{\prime \prime}\right)^{2}} .
$$

An analogous computation of $\mathcal{X}_{B}^{+}$considers instead figure 15 in the opposite resolution, that is, with the saddle trajectories interchanged, and correspondingly modified bases of sections "in between" the saddle trajectories. This yields

$$
\mathcal{X}_{B}^{+}=\frac{s_{2}^{\prime} \wedge s_{1}^{\prime}}{s_{2}^{\prime \prime} \wedge s_{1}^{\prime}} \frac{s_{2}^{\prime \prime} \wedge s_{1}^{\prime \prime}}{s_{2} \wedge s_{1}^{\prime \prime}} \frac{s_{2} \wedge \mathrm{M} s_{2}}{s_{1}^{\prime \prime} \wedge \mathrm{M} s_{2}} \frac{s_{1}^{\prime \prime} \wedge s_{2}^{\prime \prime}}{s_{2}^{\prime} \wedge s_{2}^{\prime \prime}}=\mu^{-2} \frac{\left(s_{1} \wedge s_{2}\right)\left(s_{1}^{\prime} \wedge s_{2}^{\prime}\right)\left(s_{1}^{\prime \prime} \wedge s_{2}^{\prime \prime}\right)^{2}}{\left(s_{1} \wedge s_{1}^{\prime \prime}\right)^{2}\left(s_{1}^{\prime} \wedge s_{2}^{\prime \prime}\right)^{2}} .
$$

Following the averaging prescription of $[74,77]$, we may thus associate the average $B$-cycle coordinate

$$
\mathcal{X}_{B}=\sqrt{\mathcal{X}_{B}^{-} \mathcal{X}_{B}^{+}}=\frac{s_{1}^{\prime} \wedge s_{1}^{\prime \prime}}{s_{1} \wedge s_{1}^{\prime \prime}} \frac{s_{1} \wedge s_{2}^{\prime \prime}}{s_{1}^{\prime} \wedge s_{2}^{\prime \prime}}
$$

to the Fenchel-Nielsen network $\mathscr{W}$ at the critical phase (in particular, this agrees with the computation in [77, appendix A]). 
Example 12. A construction analogous to example 11 for $K=2$ in the case of a FenchelNielson network on a surface $C$ with only regular punctures yields a complexified version of the well-known Fenchel-Nielsen coordinates. Indeed, one finds that there are two spectral coordinates $\mathcal{X}_{A}$ and $\mathcal{X}_{B}$ associated to each pants cycle in the pants decomposition of $C$, dual to the Fenchel-Nielsen network $\mathscr{W}$. Here $\mathcal{X}_{A}$ is an eigenvalue of the monodromy of $\nabla$ along the pants cycle, while $\mathcal{X}_{B}$ is characterized by a certain property under what is called the 'twist flow' on the moduli space of suitably framed flat $S L(2, \mathbb{C})$ connections on $C$ (details are found in [75, section 8.4]). These are exponentiated and complexified versions of the original length and twist coordinates introduced in the context of hyperbolic geometry, which for instance played an important role in Kontsevich's proof [93] of Witten's conjecture on the intersection numbers of the moduli space of curves [139].

The spectral coordinates obtained in example 11 are examples of Fenchel-Nielsen type coordinates on a Riemann surface with irregular punctures. Higher rank generalizations of Fenchel-Nielsen length-twist coordinates have been obtained through abelianization in [74], ${ }^{14}$ while higher rank Fenchel-Nielsen type coordinates on the three-punctured sphere have been introduced and studied in [77] by abelianization with respect to the circular Fenchel-Nielsen network in figure $11 .^{15}$

\subsection{Nonabelianization}

It is an interesting exercise to express the monodromies of a flat $\operatorname{SL}(K, \mathbb{C})$ connection $\nabla$ in terms of the data of the $\mathrm{GL}(1, \mathbb{C})$ connection $\nabla^{\mathrm{ab}}$. This is called nonabelianization [58]. It enables one to find the monodromy representation of $\nabla$ in terms of spectral coordinates $\mathcal{X}_{\gamma}$.

Example 13. Returning to example 8, let us describe the corresponding nonabelianization. For this, we suppose instead that $\nabla_{ \pm}^{\text {ab }}$ is the resulting GL(1, $\left.\mathbb{C}\right)$ connection on $\Sigma$. Then we may construct the $\mathrm{SL}(2, \mathbb{C})$ monodromies of $\nabla$ in terms of the parallel transport of $\nabla_{ \pm}^{\text {ab }}$ as follows. Consider the path groupoid depicted in figure 19. Associate a diagonal $\operatorname{SL}(2, \mathbb{C})$ matrix $\mathrm{D}_{i}$ to each path $\varrho_{i}$ that does not cross a branch cut, and an off-diagonal $\operatorname{SL}(2, \mathbb{C})$ matrix $\tilde{\mathrm{D}}_{i}$ if the path $\varrho_{i}$ crosses a branch cut. We also associate a unipotent matrix $\mathrm{S}_{k}$ with non-zero entry at position $j i$ to each trajectory of type $i j$.

Let the entries of the matrices

$$
\mathrm{D}_{i}=\left(\begin{array}{cc}
d_{i} & 0 \\
0 & d_{i}^{-1}
\end{array}\right) \quad \text { and } \quad \tilde{\mathrm{D}}_{i}=\left(\begin{array}{cc}
0 & -\tilde{d}_{i} \\
\tilde{d}_{i}^{-1} & 0
\end{array}\right)
$$

encode the parallel transport of the GL $(1, \mathbb{C})$ connection $\nabla_{ \pm}^{\mathrm{ab}}$ along lifts of the paths $\varrho_{i}$ to $\Sigma$. That is, we choose the non-zero elements $d_{i}$ and $\tilde{d}_{i}$ of the matrices $\mathrm{D}_{i}$ and $\tilde{\mathrm{D}}_{i}$ as

$$
d_{1}=\frac{g_{1}}{g_{2}}, \quad \tilde{d}_{2}=g_{1} g_{2} \mathcal{X}_{B}, \quad d_{3}=\frac{g_{3}}{g_{2}}, \quad d_{4}=\frac{g_{4}}{g_{3}}, \quad \tilde{d}_{5}=g_{3} g_{4}, \quad d_{6}=\frac{1}{\mathcal{X}_{A}},
$$

for some complex numbers $g_{1}, g_{2}, g_{3}, g_{4}$, where $\mathcal{X}_{A}$ and $\mathcal{X}_{B}$ are the spectral coordinates computed in example 11.

\footnotetext{
${ }^{14}$ Another proposal for SL(3, C) Darboux coordinates on the four-punctured sphere is found in [85].

${ }^{15}$ Other accounts and examples of abelianization may be found in e.g. [36, 118, 144].
} 


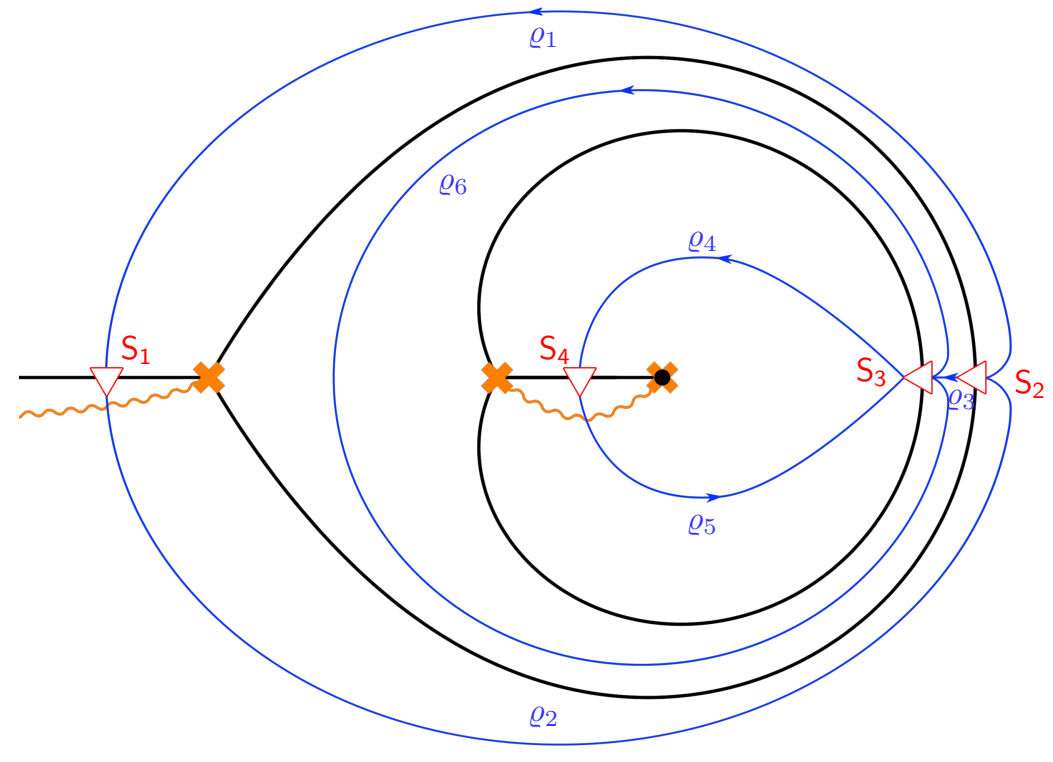

Figure 19. A choice of paths $\varrho_{i}$ on $C=\mathbb{C}^{\times}$for the network $\mathscr{W}$. Each path begins and ends at a trajectory of the network on an arrow which is labeled by a matrix $S_{k}$.

The unipotent transformations are of the form

$$
\mathrm{S}_{1}=\left(\begin{array}{cc}
1 & f_{1} \\
0 & 1
\end{array}\right), \quad \mathrm{S}_{2}=\left(\begin{array}{cc}
1 & 0 \\
f_{2} & 1
\end{array}\right)\left(\begin{array}{ll}
1 & f_{3} \\
0 & 1
\end{array}\right), \quad \mathrm{S}_{3}=\left(\begin{array}{cc}
1 & 0 \\
f_{4} & 1
\end{array}\right)\left(\begin{array}{ll}
1 & f_{5} \\
0 & 1
\end{array}\right), \quad \mathrm{S}_{4}=\left(\begin{array}{ll}
1 & f_{6} \\
0 & 1
\end{array}\right)
$$

or

$$
\mathrm{S}_{1}=\left(\begin{array}{cc}
1 & \tilde{f}_{1} \\
0 & 1
\end{array}\right), \quad \mathrm{S}_{2}=\left(\begin{array}{cc}
1 & \tilde{f}_{2} \\
0 & 1
\end{array}\right)\left(\begin{array}{cc}
1 & 0 \\
\tilde{f}_{3} & 1
\end{array}\right), \quad \mathrm{S}_{3}=\left(\begin{array}{cc}
1 & \tilde{f}_{4} \\
0 & 1
\end{array}\right)\left(\begin{array}{cc}
1 & 0 \\
\tilde{f}_{5} & 1
\end{array}\right), \quad \mathrm{S}_{4}=\left(\begin{array}{cc}
1 & \tilde{f}_{6} \\
0 & 1
\end{array}\right)
$$

for the resolution $\mathscr{W}^{+}$or $\mathscr{W}^{-}$, respectively. Solving for the off-diagonal elements $f_{k}$ and $\tilde{f}_{k}$, by requiring that the $\operatorname{SL}(2, \mathbb{C})$ monodromy around the branch points on $C$ is equal to $\nVdash$, yields

$$
\begin{array}{rlrl}
f_{1} & =-\frac{\mathcal{X}_{B}}{\mathcal{X}_{A}}\left(1+\mathcal{X}_{A}^{2}\right) g_{1}^{2}=\tilde{f}_{1}, & \\
f_{2} & =\frac{\mathcal{X}_{A}}{\mathcal{X}_{B} g_{2}^{2}} \frac{1}{1-\mathcal{X}_{A}^{2}}, & \tilde{f}_{2} & =-\frac{1}{\mathcal{X}_{A} \mathcal{X}_{B} g_{2}^{2}}, \\
f_{3} & =-\frac{\mathcal{X}_{A}}{\mathcal{X}_{B}} g_{2}^{2}, & \tilde{f}_{3} & =\frac{\mathcal{X}_{A} \mathcal{X}_{B} g_{2}^{2}}{1-\mathcal{X}_{A}^{2}}, \\
f_{4} & =\frac{1}{\mathcal{X}_{A} g_{3}^{2}}, & \tilde{f}_{4} & =\frac{g_{3}^{2}}{\mathcal{X}_{A}}, \\
f_{5} & =-\frac{\mathcal{X}_{A} g_{3}^{2}}{1-\mathcal{X}_{A}^{2}}, & \tilde{f}_{5} & =-\frac{\mathcal{X}_{A}}{g_{3}^{2}\left(1-\mathcal{X}_{A}^{2}\right)}, \\
f_{6} & =-\frac{g_{4}^{2}}{\mathcal{X}_{A}}\left(1+\mathcal{X}_{A}^{2}\right)=\tilde{f}_{6} . &
\end{array}
$$


Then the monodromies of the $\mathrm{SL}(2, \mathbb{C})$ connection $\nabla$ along any path on $C$, written as a concatenation of the paths $\varrho_{i}$, are given by multiplying the corresponding matrices $\mathrm{D}_{i}, \tilde{\mathrm{D}}_{i}$ and $\mathrm{S}_{k}$. Notice that although there are various other parameters $g_{i}$ around (corresponding to an abelian gauge choice), the monodromy invariants of $\nabla$ can be expressed entirely in terms of the spectral coordinates $\mathcal{X}_{A}$ and $\mathcal{X}_{B}$.

A final point worth mentioning here is that the off-diagonal elements $f_{k}$ of the Stokes matrices $S_{k}$ have an interpretation in terms of the GL(1, C) connection $\nabla_{ \pm}^{\text {ab }}$ as well: they represent the parallel transport of $\nabla_{ \pm}^{\text {ab }}$ along "detour paths" on $\Sigma$ that follow the corresponding trajectory back to the branch point they are emitted from. Multiple terms in $f_{k}$ correspond to multiple trajectories in the network. For example, each term in the power series expansion

$$
f_{2}=\frac{\mathcal{X}_{A}}{\mathcal{X}_{B} g_{2}^{2}}\left(1+\mathcal{X}_{A}^{2}+\mathcal{X}_{A}^{4}+\cdots\right)
$$

corresponds to the parallel transport of $\nabla_{ \pm}^{\text {ab }}$ along a detour path which encircles the $A$-cycle on $\Sigma$ multiple times.

A more thorough description of nonabelianization (and its relation to abelianization) can be found in [75], where nonabelianization is shown to be one-to-one for the FockGoncharov and Fenchel-Nielsen type networks in rank $K=2$. However, this is not always the case, see for instance the discussion in [77] on nonabelianization for the higher rank Fenchel-Nielsen networks on the three-punctured sphere, illustrated in figure 11. Nonabelianization is extended to other Lie groups in [79].

\section{Theories of class $\mathcal{S}$ and their BPS states}

In this section we introduce class $\mathcal{S}$ theories, which are a rich subclass of $\mathcal{N}=2$ supersymmetric field theories in four dimensions. We start with a brief introduction to fourdimensional $\mathcal{N}=2$ supersymmetric field theories. In section 3.1 we go back to the seminal work of Seiberg and Witten [123, 124], introducing Seiberg-Witten geometry; ${ }^{16}$ here we follow the reviews $[39,95]$, see also [98] for a more recent account. ${ }^{17}$ In section 3.2 we specialize to a class of four-dimensional $\mathcal{N}=2$ theories known as class $\mathcal{S}$ theories, which was first discovered by Gaiotto [54]; for more extensive reviews see e.g. [106, 122, 131, 135]. Finally, in section 3.3 we make contact with the spectral networks introduced in section 2 . We explain how the BPS spectra of class $\mathcal{S}$ theories may be obtained from spectral networks, particularly for the example of Seiberg-Witten theory; more details about this can be found in [58].

\subsection{Seiberg-Witten geometry}

A four-dimensional $\mathcal{N}=2$ theory is a supersymmetric field theory on $\mathbb{R}^{3,1}$ which is invariant under two supercharges in the fundamental representation of $\operatorname{Spin}(3,1) \cong \operatorname{SL}(2, \mathbb{C})$, or

\footnotetext{
${ }^{16}$ However, we do not review the most physically interesting idea of Seiberg-Witten theory, which is the relation to monopole condensation and confinement.

${ }^{17}$ We further recommend the lectures of Witten for an introduction to Seiberg-Witten theory, which are available at https://www.youtube.com/watch?v=EC1SvnjYWsA and https://www.youtube.com/ watch? $=9 \mathrm{nPU} 3 \mathrm{WNhH}-0$.
} 
equivalently under eight real supercharges. One usually writes them as $Q_{\alpha}^{I}$ and $\bar{Q}_{\dot{\alpha}}^{I}$, where $I=1,2$ counts the supercharges (they are called R-symmetry indices: the $\mathrm{SU}(2)_{R} \subset$ $\mathrm{SL}(2, \mathbb{C})$ R-symmetry rotates them), and $\alpha, \dot{\alpha}=1,2$ are spinor indices. There are two different particle multiplets in an $\mathcal{N}=2$ gauge theory. The vector multiplet consists of a gauge field $A_{\mu}$, two spinors $\lambda_{\alpha}^{I}$ and $\bar{\lambda}_{\dot{\alpha}}^{I}$, and a complex scalar $\phi$. All of them transform in the adjoint representation of the gauge group $G$ (or more precisely the gauge algebra $\mathfrak{g}$ ). The other multiplet is the hypermultiplet. It consists of a pair of complex scalars $q^{I}$, and two spinors $\psi_{\alpha}^{I}$ and $\bar{\psi}_{\dot{\alpha}}^{I}$. These may transform in any representation of the gauge group $G$ (or more precisely $\mathfrak{g}$ ). ${ }^{18}$ The hypermultiplet representation can sometimes be reduced to half of its degrees of freedom, which is then called a half-hypermultiplet.

We start by studying the pure $\mathrm{SU}(2)$ gauge theory which just consists of a single vector multiplet in the adjoint representation of $\mathfrak{g}=\mathfrak{s u}(2)$. The Lagrangian includes a potential term for the scalar field $\phi$ which is given by

$$
V(\phi)=\operatorname{Tr}\left[\phi, \phi^{\dagger}\right]^{2} .
$$

This potential vanishes if $\phi$ is restricted to the complexified maximal torus of SU(2), i.e.

$$
\phi=a \sigma_{3}
$$

where $a \in \mathbb{C}$ and $\sigma_{3}$ generates the maximal torus of $\mathrm{SU}(2)$. The potential $V(\phi)$ thus has flat directions or moduli. Physically distinct vacua are parametrized by the gauge invariant Casimir element

$$
u(a)=\operatorname{Tr} \phi^{2},
$$

so that the classical moduli space of vacua is $\mathscr{B}_{\mathrm{c}} \cong \mathbb{P}^{1}$ (if we add a point at infinity). The space $\mathscr{B}_{c}$ is also called the (classical) Coulomb branch. Indeed, if $u \neq 0$ then all fields will acquire masses by the Higgs mechanism, except for the component of the vector multiplet in the direction of $\phi$. After integrating out the massive fields (after all, we are interested in the infrared physics), we are left with an effective abelian theory. This is obviously not possible at $u=0$ and $u=\infty$, and we therefore regard these two points as singularities of $\mathscr{B}_{\mathrm{c}}$.

The effective abelian theory is characterized by a prepotential $\mathcal{F}_{0}(a)$. The complexified gauge coupling

$$
\tau(a)=\frac{\theta}{\pi}+\frac{8 \pi \mathrm{i}}{g_{\mathrm{YM}}^{2}}
$$

is given as its second derivative

$$
\tau(a)=\frac{\partial^{2} \mathcal{F}_{0}(a)}{\partial a^{2}}
$$

Furthermore, the pair

$$
\Omega=\left(\begin{array}{c}
a_{\mathrm{D}} \\
a
\end{array}\right)
$$

\footnotetext{
${ }^{18}$ More precisely, $q^{1}$ and $q^{2}$ live in different representations of $G$ which are conjugate to each other, and similarly for $\psi_{\alpha}^{I}$ and $\bar{\psi}_{\dot{\alpha}}^{I}$.
} 
where

$$
a_{\mathrm{D}}=\frac{\partial \mathcal{F}_{0}(a)}{\partial a},
$$

is known to define a holomorphic section of an $\operatorname{Sp}(2, \mathbb{Z}) \cong \mathrm{SL}(2, \mathbb{Z})$ vector bundle over the Coulomb branch $\mathscr{B}_{\mathrm{c}}$. Seiberg and Witten have shown us how to find an explicit description of the quantum moduli space $\mathscr{B}=\mathscr{B}_{\mathrm{q}}$, i.e. an explicit description of this $\mathrm{SL}(2, \mathbb{Z})$ bundle [123].

The starting point for the Seiberg-Witten solution is the behaviour of $\mathcal{F}_{0}(a)$ around $u=\infty$, where the leading contribution can be computed in perturbation theory. The coupling constant has an expansion

$$
\tau(a)=\tau_{0}+\frac{\mathrm{i}}{\pi} \log \left(\frac{a}{\Lambda^{2}}\right)+\sum_{n=1}^{\infty} c_{n}\left(\frac{\Lambda}{a}\right)^{4 n},
$$

where the first term $\tau_{0}$ is the classical contribution, the second term is the one-loop contribution (this is the only perturbative correction), and the remaining terms are instanton corrections. The ultraviolet cutoff $\Lambda$ is exponentially small from the classical point of view, so that quantum mechanically the single classical singularity there splits in two. We will say more about this expansion in section 4 .

What is important here is that the logarithmic contributions determine the local monodromy of the $\mathrm{SL}(2, \mathbb{Z})$ bundle around $u=\infty$. If we follow a loop around $u=\infty$ we find the transformation

$$
\left(\begin{array}{c}
a_{\mathrm{D}} \\
a
\end{array}\right) \longmapsto\left(\begin{array}{c}
-a_{\mathrm{D}}+2 a \\
-a
\end{array}\right)=\mathrm{M}_{\infty}\left(\begin{array}{c}
a_{\mathrm{D}} \\
a
\end{array}\right) \quad \text { with } \quad \mathrm{M}_{\infty}=\left(\begin{array}{cc}
-1 & 2 \\
0 & -1
\end{array}\right)
$$

The simplest solution would be to extend the $\operatorname{SL}(2, \mathbb{Z})$ vector bundle over $\mathbb{P}^{1}$ by having the opposite monodromy around the origin $u=0$. This however turns out to violate the positivity constraint $\operatorname{Im}(\tau)=8 \pi / g_{\mathrm{YM}}^{2}>0$. The Seiberg-Witten solution assumes the minimal amount of a total of three singularities, say at $u=\infty$ and $u= \pm \Lambda^{2}$, with monodromy matrices generating a subgroup $\Gamma \subset \mathrm{SL}(2, \mathbb{Z})$. The quantum moduli space is then given by $\mathscr{B}=\mathbb{H} / \Gamma$, where $\mathbb{H}$ is the upper complex half-plane.

This moduli space also happens to parametrize elliptic curves of the form

$$
\Sigma: \quad w^{2}=\frac{\Lambda^{2}}{z^{3}}-\frac{2 u}{z^{2}}+\frac{\Lambda^{2}}{z},
$$

now known as Seiberg-Witten curves, ${ }^{19}$ where the first homology of $\bar{\Sigma}$ may be identified with the charge lattice $\Gamma$ of the $\operatorname{SL}(2, \mathbb{Z})$ bundle:

$$
\Gamma=H_{1}(\bar{\Sigma}, \mathbb{Z}) .
$$

Note that we have already encountered the family of curves $\Sigma$ (with $\Lambda=1$ and $u=-\frac{9}{8}$ ) in section 2, see examples $3,8,11$ and 13 , as a branched covering $\Sigma \rightarrow C$ defined by the quadratic differential $p_{2}(z)$ from (2.10). From the Riemann-Hurwitz formula it follows that $\Sigma$ is topologically a torus with two double punctures, see figure 20.

\footnotetext{
${ }^{19}$ This is a different formulation of $\Sigma$ compared to the original formulation given by Seiberg and Witten. It is the more recent class $\mathcal{S}$ theory formulation [54], which is needed to apply spectral network techniques.
} 

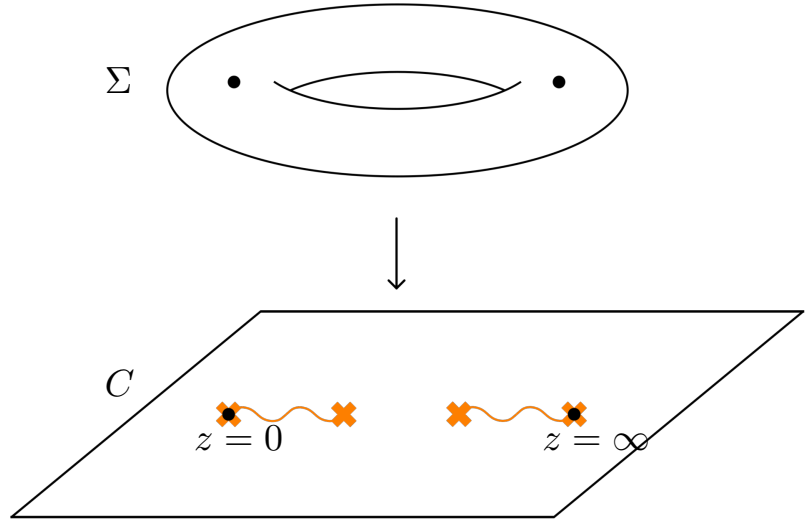

Figure 20. Seiberg-Witten geometry for the pure $\mathrm{SU}(2)$ theory. The ultraviolet curve $C$ is a sphere with irregular singularities (of order three) at $z=0$ and $z=\infty$, while the Seiberg-Witten curve $\Sigma$ is topologically a torus with two double punctures.

Seiberg and Witten discovered that this geometry is crucial for understanding the infrared properties of the pure $\mathrm{SU}(2)$ gauge theory. Indeed, one can show that the moduli $a$ and $a_{\mathrm{D}}$ are given by period integrals

$$
a=\oint_{A} \lambda \quad \text { and } \quad a_{\mathrm{D}}=\oint_{B} \lambda,
$$

where $(A, B)$ is a canonical basis of $H_{1}(\Sigma, \mathbb{Z})$ (see figure 21) and $\lambda$ is the unique one-form on $\Sigma$ given by

$$
\lambda=w \mathrm{~d} z
$$

which is called the Seiberg-Witten differential. The singularities at $u=\infty$ and $u= \pm \Lambda^{2}$ on $\mathscr{B}$ are then the points in the moduli space where the Seiberg-Witten curve $\Sigma$ degenerates, and some combination of one-cycles vanishes. As we will see later in section 3.3, one can read off the BPS spectrum of the field theory from the Seiberg-Witten curve. The singularities correspond to moduli where certain BPS states become massless: $W^{ \pm}$-bosons become massless at $u=\infty$ (restoring the $\mathrm{SU}(2)$ symmetry), whereas certain dyons become massless at $u= \pm \Lambda^{2}$.

The Seiberg-Witten solution was based on the premise that the abelian gauge field was the only massless field in the theory. Thus we expect the formalism to break down at $u=\infty$ and $u= \pm \Lambda^{2}$, where there are additional massless BPS particles (that could not be integrated out). Reinstating these massless states removes these singularities. The singularities on $\mathscr{B}$ are therefore not physical.

Following the works of Seiberg and Witten, similar techniques have been used to find Seiberg-Witten curves for many other Lagrangian $\mathcal{N}=2$ theories. More importantly, it was realized that Seiberg-Witten curves are not just auxiliary mathematical objects which are helpful in the study of $\mathcal{N}=2$ gauge theories, but they can actually be identified as physical objects appearing in various string theory constructions of $\mathcal{N}=2$ theories, see e.g. $[16,90,142]$. Perhaps the most insightful construction is obtained by considering M5branes in the M-theory background $\mathbb{R}^{3,1} \times X \times \mathbb{R}^{3}$, with an M5-brane wrapping $\mathbb{R}^{3,1} \times \Sigma \times\{0\}$, 

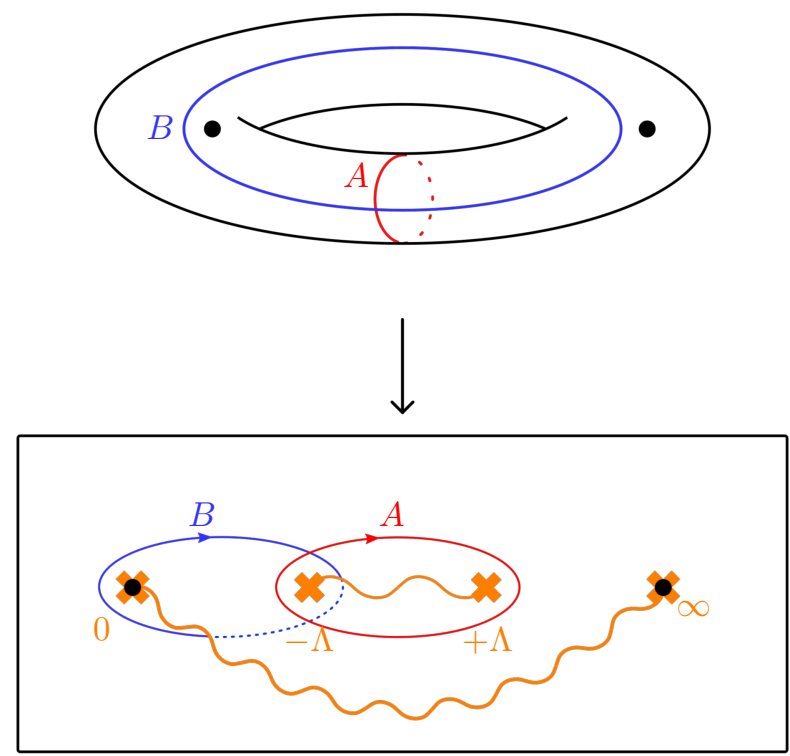

Figure 21. Canonical basis $(A, B)$ of one-cycles on $\Sigma$ viewed as a two-sheeted branched cover of a base curve $C$.

where $\Sigma \subset X$ is the Seiberg-Witten curve embedded in an ambient four-dimensional space $X$ and $\{0\} \subset \mathbb{R}^{3}$ is the origin of $\mathbb{R}^{3}$. This realizes the corresponding $\mathcal{N}=2$ theory as the worldvolume theory on the M5-brane.

\subsection{Class $\mathcal{S}$ geometry}

So far we have emphasized the feature that the low energy properties of any $\mathcal{N}=2$ field theory are encoded in a geometrical object, the Seiberg-Witten curve $\Sigma$, together with the Seiberg-Witten differential $\lambda$. From now on we will specialize to a subclass of $\mathcal{N}=2$ theories for which the Seiberg-Witten curve $\Sigma$ comes with some additional structure. Namely, $\Sigma$ is a (possibly branched) cover of another (possibly punctured) Riemann surface $C$, where $\Sigma \subset T^{*} C$ and $\lambda$ is the tautological one-form on the cotangent bundle $T^{*} C$ restricted to $\Sigma$. In contrast to $\Sigma$, the ultraviolet curve $C$, together with some additional data at its punctures, encodes the microscopic properties of the $\mathcal{N}=2$ field theory, and it defines the $\mathcal{N}=2$ theory uniquely. These $\mathcal{N}=2$ theories are called class $\mathcal{S}$ theories [54]. Note that this is the geometric setup of section 2, where we considered $K$-fold coverings $\Sigma \rightarrow C$ defined by a collection of higher differentials on $C$. Here the integer $K-1$ corresponds to the rank of the gauge algebra $\mathfrak{g}$ of the $\mathcal{N}=2$ theory, so that $K=2$ sheeted coverings describe $\mathrm{SU}(2)$ gauge theories.

The complex structure moduli of $C$ correspond to complexified gauge couplings of the $\mathcal{N}=2$ theory, which is often a quiver gauge theory consisting of various gauge theories coupled to one another using matter fields. The additional data needed at the higher rank punctures is determined by the type of singularities of the differentials. Regular singularities are characterized by Lie algebra homomorphisms $\varrho_{i}: \mathfrak{s u}(2) \rightarrow \mathfrak{g}$. Roughly speaking, they encode the flavour symmetries of the $\mathcal{N}=2$ theory, which are the global 

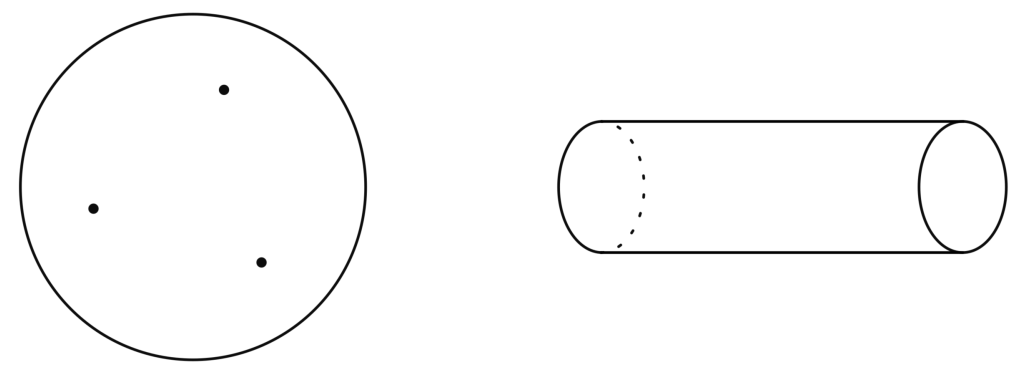

Figure 22. The three punctured sphere and the cylinder which constitute the building blocks for $\mathrm{SU}(2)$ class $\mathcal{S}$ theories.

symmetries acting on the matter fields of the $\mathcal{N}=2$ theory, with group $G^{Q_{i}}$ given by the centralizer of $\varrho_{i}$ in the gauge group $G$. We will take the gauge algebra to be $\mathfrak{g}=\mathfrak{s u}(K)$, and we denote the corresponding $\mathcal{N}=2$ theory of class $\mathcal{S}$ by $\mathrm{T}_{K}[C, \mathcal{D}]$, where $\mathcal{D}$ represents the additional data at the punctures, which in this case correspond to Young diagrams with $K$ boxes. The theory $\mathrm{T}_{K}[C, \mathcal{D}]$ may be realized physically as the worldvolume theory of $K$ M5-branes wrapped on $\mathbb{R}^{3,1} \times C \times\{0\}$ in the M-theory background $\mathbb{R}^{3,1} \times T^{*} C \times \mathbb{R}^{3}$, or equivalently as a (twisted) compactification of the six-dimensional $(2,0)$-theory $\mathfrak{X}[K]$ on the Riemann surface $C$.

Class $\mathcal{S}$ theories of type $\mathbf{S U}(2)$. Conformal $\mathrm{SU}(2)$ theories of class $\mathcal{S}$ can be constructed out of just two elementary building blocks (see figure 22):

- the three-punctured sphere with an associated $\mathrm{SU}(2)$ flavour symmetry group at each puncture (this is only possible for $K=2$ as there is only a single Young diagram with two boxes at each puncture); and

- the cylinder with complex structure parameter

$$
\tau_{\mathrm{UV}}=\frac{\theta}{2 \pi}+\mathrm{i} \frac{4 \pi}{g_{\mathrm{YM}}^{2}},
$$

where $g_{\mathrm{YM}}$ is real and $\theta$ is periodic with period $2 \pi$.

The cylinder corresponds to an $\mathcal{N}=2$ vector multiplet with gauge group $\mathrm{SU}(2)$ and complexified gauge coupling $\tau_{\mathrm{UV}}$, while the three-punctured sphere corresponds to a halfhypermultiplet in the trifundamental representation of $\mathrm{SU}(2)_{a} \times \mathrm{SU}(2)_{b} \times \mathrm{SU}(2)_{c}$ (see $[73$, 132] for further details about this half-hypermultiplet as well as a Lagrangian description). All theories that are constructed using these building blocks have a Lagrangian description and may be checked to be conformal (if all masses are set to zero). This means in particular that the complexified coupling $\tau_{\mathrm{UV}}$ is a dimensionless parameter. (However, in contrast to $\mathcal{N}=4$ supersymmetric Yang-Mills theory we should differentiate here between the (exactly marginal) microscopic coupling $\tau_{\mathrm{UV}}$ and the infrared coupling $\tau$.)

For example, we may construct the $\mathrm{SU}(2)$ gauge theory coupled to four additional fundamental hypermultiplets by gluing two three-punctured spheres using the plumbing fixture method. For this, pick a puncture on each sphere, with the first puncture at $z=0$ 


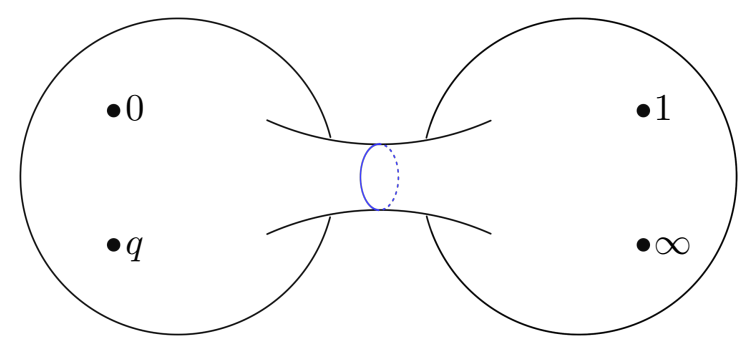

Figure 23. Plumbing fixture construction of the four-punctured sphere.

in a local coordinate $z$ and the second puncture at $w=0$ in a local coordinate $w$. Then make the identification $z w=q$, where

$$
q=\mathrm{e}^{2 \pi \mathrm{i} \tau_{\mathrm{UV}}}
$$

is the exponentiated gauge coupling (see figure 23). Indeed, on the gauge theory side this means coupling the two corresponding $\mathrm{SU}(2)$ flavour symmetries to a dynamical $\mathrm{SU}(2)$ gauge field with complexified coupling $\tau_{\mathrm{UV}}$. The four leftover $\mathrm{SU}(2)$ flavour symmetries combine to give the enhanced $\mathrm{SO}(8)$ flavour symmetry group of the $\mathrm{SU}(2)$ gauge theory coupled to four hypermultiplets. Note that, in general, the Lagrangian for a theory with four hypermultiplets in a complex representation of the gauge group is invariant under an $\mathrm{SU}(4)$ flavour symmetry rotating the four hypermultiplets. However, the case of $\mathrm{SU}(2)$ gauge group is special, since its fundamental representation is pseudoreal. This implies that the flavour symmetry is in fact enhanced to an $\mathrm{SO}(8)$ flavour symmetry which rotates the eight half-hypermultiplets. We can embed the flavour symmetries $\mathrm{SU}(2)_{i}$ acting on the individual hypermultiplets into this flavour symmetry group:

$$
\mathrm{SO}(8) \supset \mathrm{SU}(2)_{a} \times \mathrm{SU}(2)_{b} \times \mathrm{SU}(2)_{c} \times \mathrm{SU}(2)_{d} .
$$

Changing the complex structure parameter of the four-punctured sphere changes the complexified gauge coupling $\tau_{\mathrm{UV}}$. But we can ask about the effect of a $\operatorname{PSL}(2, \mathbb{Z})$ action on $\tau_{\mathrm{UV}}$ given by

$$
\tau_{\mathrm{UV}} \longmapsto \frac{a \tau_{\mathrm{UV}}+b}{c \tau_{\mathrm{UV}}+d} \quad \text { for } \quad\left(\begin{array}{ll}
a & b \\
c & d
\end{array}\right) \in \mathrm{SL}(2, \mathbb{Z}) .
$$

This action leaves the complex structure of the four-punctured sphere invariant and just permutes the punctures. It suggests that when the $\mathrm{SU}(2)$ gauge theory with $N_{\mathrm{f}}=4$ flavour hypermultiplets is strongly coupled, that is when $q \rightarrow 1$ or $q \rightarrow \infty$, we can equivalently describe it as a weakly coupled theory with $q \rightarrow 0$ and permuted flavour symmetry groups (see figure 24). This is indeed the S-duality found in the $\mathrm{SU}(2)$ gauge theory coupled to four hypermultiplets by Argyres and Seiberg [13]. In [54] this S-duality was generalized to any theory of class $\mathcal{S}$ : any theory $\mathrm{T}_{K}[C, \mathcal{D}]$ is invariant under a generalized S-duality that is realized geometrically as the mapping class group acting on the ultraviolet curve $C$. This is a main reason for naming them theories of class $\mathcal{S}$.

Let us finally relate this discussion to section 3.1 by noting that any asymptotically free theory of class $\mathcal{S}$ may be realized in this framework by sending some masses to infinity. 

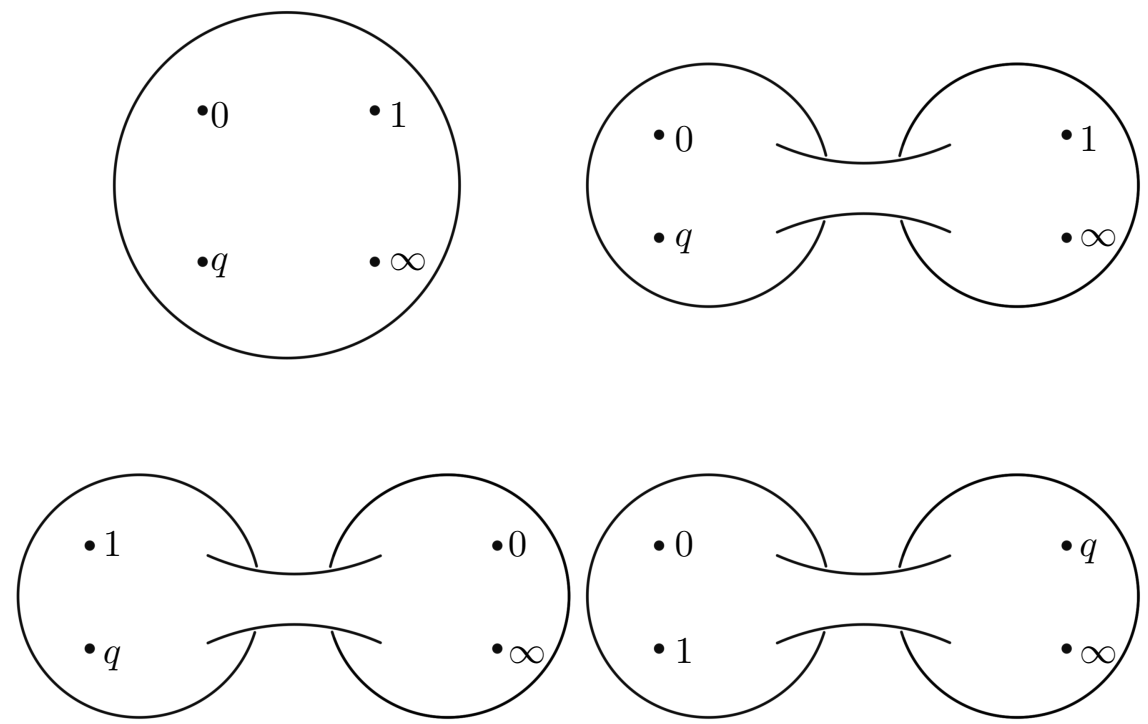

Figure 24. The four-punctured sphere and its limits when $q \rightarrow 0,1, \infty$.

For instance, the pure $\mathrm{SU}(2)$ gauge theory is obtained by sending all four masses $m_{i}$ of the hypermultiplets in the $N_{\mathrm{f}}=4 \mathrm{SU}(2)$ theory to infinity, leaving $q=\mathrm{e}^{2 \pi \mathrm{i} \tau_{\mathrm{UV}}}=$ $\Lambda^{4} / m_{1} m_{2} m_{3} m_{4}$ finite, where $\Lambda$ is the ultraviolet scale of the pure $\mathrm{SU}(2)$ theory. The resulting ultraviolet curve $C$ is a sphere with two irregular punctures. The corresponding Seiberg-Witten geometry is described by the quadratic differential

$$
p_{2}(z)=\left(\frac{\Lambda^{2}}{z^{3}}-\frac{2 u}{z^{2}}+\frac{\Lambda^{2}}{z}\right) \mathrm{d} z \otimes \mathrm{d} z,
$$

as anticipated from (3.10).

Class $\mathcal{S}$ theories of type $\mathbf{S U}(\boldsymbol{K})$. As for spectral networks, the story changes quite drastically for higher rank gauge groups $\mathrm{SU}(K)$ with $K>2$. Conformal $\mathrm{SU}(K)$ theories of class $\mathcal{S}$ can again be built out of three-punctured spheres and cylinders which are now labeled by Young diagrams with $K$ boxes. However, the corresponding $\mathcal{N}=2$ field theories are often "non-Lagrangian", meaning that they are intrinsically stongly coupled quantum field theories which do not admit a weakly coupled description that can be studied using perturbation theory. ${ }^{20}$

As an example, let us consider $K=3$. Class $\mathcal{S}$ theories of type $\mathrm{SU}(3)$ can be constructed out of three elementary building blocks (see figure 25):

- the three-punctured sphere with one "minimal" and two "maximal" punctures;

- the three-punctured sphere with three "maximal" punctures; and

- the cylinder with complex structure parameter $\tau_{\mathrm{UV}}$ labeled by two "maximal" punctures.

\footnotetext{
${ }^{20}$ They can be investigated physically as infrared limits of $\mathcal{N}=1$ gauge theories, where supersymmetry becomes enhanced at the fixed points, see e.g. [53, 99].
} 


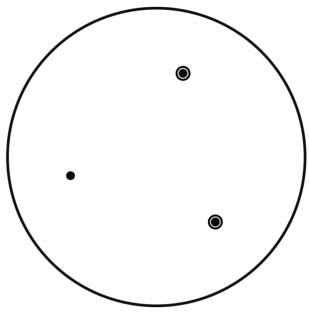

A

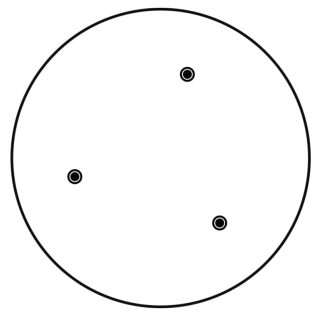

B

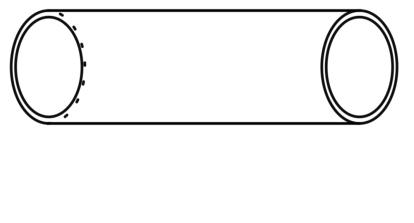

C

Figure 25. $\mathrm{SU}(3)$ class $\mathcal{S}$ theories are built from: (A) the sphere with two maximal and one minimal puncture; (B) the sphere with three maximal punctures; (C) the cylinder with two maximal punctures.

Here a maximal puncture refers to a puncture labeled by a Young diagram consisting of one row with three boxes, together with mass parameters $\left(m_{1}, m_{2},-m_{1},-m_{2}\right)$ labeling the residues of the Seiberg-Witten differential $\lambda$ at the (regular) puncture; the associated flavour symmetry group is $\mathrm{SU}(3)$ since the multiplicity of column heights is three. A minimal puncture refers to a puncture labeled by a Young diagram consisting of one column with two boxes and one column with one box, together with mass parameters $(m, m,-2 m)$; the associated flavour symmetry group is $\mathrm{U}(1)=S(\mathrm{U}(1) \times \mathrm{U}(1))$ since the multiplicities of column heights are $(1,1)$. Similarly to the rank one case $K=2$, the cylinder corresponds to an $\mathcal{N}=2$ vector multiplet with gauge group $\mathrm{SU}(3)$ and complexified coupling $\tau_{\mathrm{UV}}$, while the three-punctured sphere with two maximal and one minimal puncture now corresponds to a free hypermultiplet in the bifundamental representation of $\mathrm{SU}(3)_{a} \times \mathrm{SU}(3)_{c}$.

We may then construct the superconformal SU(3) gauge theory coupled to six hypermultiplets (this is the $\mathrm{SU}(3)$ analogue of the superconformal $N_{\mathrm{f}}=4 \mathrm{SU}(2)$ theory) by gluing two such three-punctured spheres using the plumbing fixture method; indeed, we can check that the $\mathrm{U}(6)$ flavour symmetry rotating the six hypermultiplets decomposes into

$$
\mathrm{U}(6) \supset \mathrm{U}(3) \times \mathrm{U}(3)=\left(\mathrm{SU}(3)_{a} \times \mathrm{U}(1)_{b}\right) \times\left(\mathrm{SU}(3)_{c} \times \mathrm{U}(1)_{d}\right) .
$$

This is illustrated in figure 26. Suppose the punctures are positioned at $z=0,1, q, \infty$, respectively, with $q$ close to 0 in this weakly coupled description of the $N_{\mathrm{f}}=6 \mathrm{SU}(3)$ theory. In the strongly coupled limit $q \rightarrow 1$, the ultraviolet curve can instead be described by the (degenerated) four-punctured sphere, as illustrated in figure 27 (to be precise, here we apply a mapping class group action to permute the punctures). This decomposes into a three-punctured sphere with three maximal punctures, and another three-punctured sphere with one maximal and two minimal punctures.

Gaiotto interpreted this geometry in [54] as the S-dual description of the strongly coupled $N_{\mathrm{f}}=6 \mathrm{SU}(3)$ theory. It has been argued by Argyres and Seiberg [13] that this S-dual description is given by an intrinsically strongly coupled interacting superconformal field theory, the $E_{6}$ Minahan-Nemeschansky theory [101], coupled to a weakly coupled $\mathrm{SU}(2)$ theory. In particular, the field theory description of the three-punctured sphere with three maximal punctures is given by the "non-Lagrangian" $E_{6}$ Minahan-Nemeschansky 


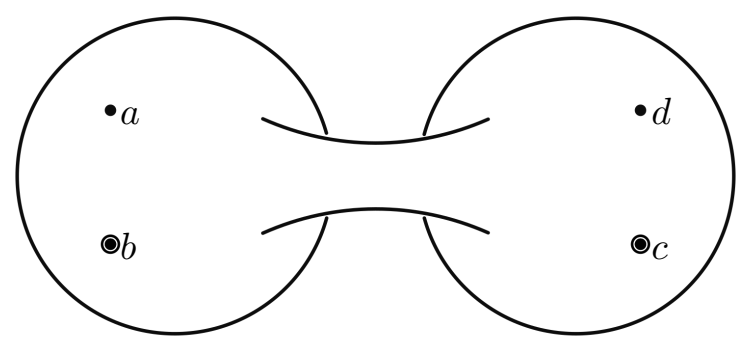

Figure 26. The ultraviolet curve for the superconformal SU(3) theory as $q \rightarrow 0$.

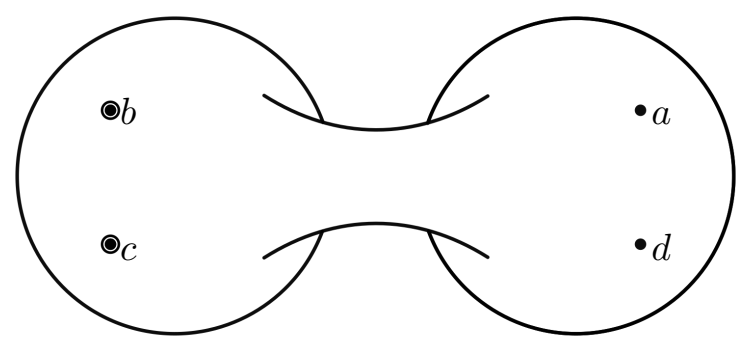

Figure 27. The ultraviolet curve for the superconformal SU(3) theory as $q \rightarrow 1$.

theory [54]. Microscopically, its flavour symmetry group is given by $\mathrm{SU}(3)_{a} \times \mathrm{SU}(3)_{b} \times$ $\mathrm{SU}(3)_{c}$, which in the low energy limit is enhanced to $E_{6}$. A classification of all the building blocks (or "tinkertoys") for theories of class $\mathcal{S}$ has been given in e.g. [31].

To summarise, there is a large class of quantum field theories $\mathrm{T}_{K}[C, \mathcal{D}]$, including most known Lagrangian ${ }^{21}$ as well as new non-Lagrangian $\mathcal{N}=2$ theories, whose microscopic data is encoded in a (possibly punctured) Riemann surface $C$. These theories may be built out of elementary blocks corresponding to three-punctured spheres and cylinders. The (quantum) Coulomb branch $\mathscr{B}$ of infrared vacua of a theory $\mathrm{T}_{K}[C, \mathcal{D}]$ is parameterized by $K$ - 1 -tuples of meromorphic $k$-differentials $\left(p_{2}, \ldots, p_{K}\right)$ on $C$, with $2 \leq k \leq K$, whose poles at the punctures are characterized by the singularity data $\mathcal{D}$; just as in section 2.2 , regular punctures correspond to poles of order $k$, and wild punctures have higher order singularities. Each tuple $\left(p_{2}, \ldots, p_{K}\right)$ defines a spectral curve

$$
\Sigma: \quad w^{K}-\sum_{k=2}^{K} p_{k} w^{K-k}=0
$$

in the cotangent bundle $T^{*} C$, with local coordinates $(z, w)$ where $z \in C$. The curve $\Sigma$ is the Seiberg-Witten curve for the theory $\mathrm{T}_{K}[C, \mathcal{D}]$ at the vacuum corresponding to $\left(p_{2}, \ldots, p_{K}\right)$. The Seiberg-Witten differential is simply the tautological one-form $\lambda=w \mathrm{~d} z$ on $T^{*} C$ restricted to $\Sigma$. Explicit descriptions of Seiberg-Witten curves and differentials for the theories discussed above may be found in e.g. [74].

\footnotetext{
${ }^{21} \mathcal{N}=2$ complete BPS quiver gauge theories were classified in [30]; there are 11 exceptional theories that are not of class $\mathcal{S}$.
} 


\subsection{BPS states from spectral networks}

In section 3.2 we introduced $\mathcal{N}=2$ theories $\mathrm{T}_{K}[C, \mathcal{D}]$ of class $\mathcal{S}$ and found that their moduli spaces of Coulomb vacua are parametrized by tuples $u=\left(p_{2}, \ldots, p_{K}\right)$ of $k$-differentials on $C$, each defining a $K$-fold (possibly branched) cover $\Sigma \rightarrow C$. We also know from section 2 that, together with a phase $\vartheta \in \mathbb{R} / 2 \pi \mathbb{Z}$, this data allows us to construct a spectral network $\mathscr{W}(u, \vartheta)$ on $C$. One may wonder at this stage whether this spectral network encodes any physical data of the theory $\mathrm{T}_{K}[C, \mathcal{D}]$ in the vacuum $u$. The answer is affirmative: the spectral network $\mathscr{W}(u, \vartheta)$ encodes in a beautiful way information about various types of BPS states [57-60]. In the following we will explain how spectral networks can be used to find the spectrum of BPS particles in the theory $\mathrm{T}_{K}[C, \mathcal{D}]$.

Any theory with extended supersymmetry has special representations, called BPS states, which are annihilated by certain linear combinations of supercharges. These BPS states have masses $M$ which saturate the BPS bound

$$
M \geq|Z|,
$$

where $Z$ is the central charge of the supersymmetry algebra. They are an important tool in understanding the theory and its dualities.

Any $\mathcal{N}=2$ field theory has half-BPS states, which are annihilated by half of the supercharges and form small representations of dimension two (rather than four). In the low energy limit, when the $\mathcal{N}=2$ theory is described by an abelian gauge theory with prepotential $\mathcal{F}_{0}(a)$, the central charge $Z$ takes the form

$$
Z=q_{i} a^{i}+p^{i} \frac{\partial \mathcal{F}_{0}(a)}{\partial a^{i}}
$$

The pair of integer vectors $(\vec{q}, \vec{p})$ labels the electric charges $\vec{q}$ and the magnetic charges $\vec{p}$ of the BPS state. The central charge can be expressed in terms of the Seiberg-Witten curve $\Sigma$ through the period integrals

$$
a^{i}=\oint_{A^{i}} \lambda \quad \text { and } \quad a_{\mathrm{D}, i}=\frac{\partial \mathcal{F}_{0}(a)}{\partial a^{i}}=\oint_{B_{i}} \lambda,
$$

where $\left(A^{i}, B_{i}\right)$ generate a symplectic basis ${ }^{22}$ of the first homology group $H_{1}(\bar{\Sigma}, \mathbb{Z})$ and $\lambda$ is the Seiberg-Witten differential. (This is a generalization of the formula (3.12) for the pure $\mathrm{SU}(2)$ gauge theory discussed in section 3.1.)

A four-dimensional BPS particle is a one-particle state in $\mathbb{R}^{3,1}$ that preserves half of the supersymmetry. Its electromagnetic charge $(\vec{q}, \vec{p})$ determines a one-cycle

$$
\gamma=q_{i} A^{i}+p^{i} B_{i}
$$

on $\Sigma$. Its central charge is then given by

$$
Z_{\gamma}=\oint_{\gamma} \lambda
$$

and its mass by $M_{\gamma}=\left|Z_{\gamma}\right|$.

\footnotetext{
${ }^{22} \mathrm{~A}$ symplectic basis is a homology basis for which the only non-zero intersection pairings are given by $\left\langle A^{i}, B_{j}\right\rangle=\delta_{j}^{i}$.
} 

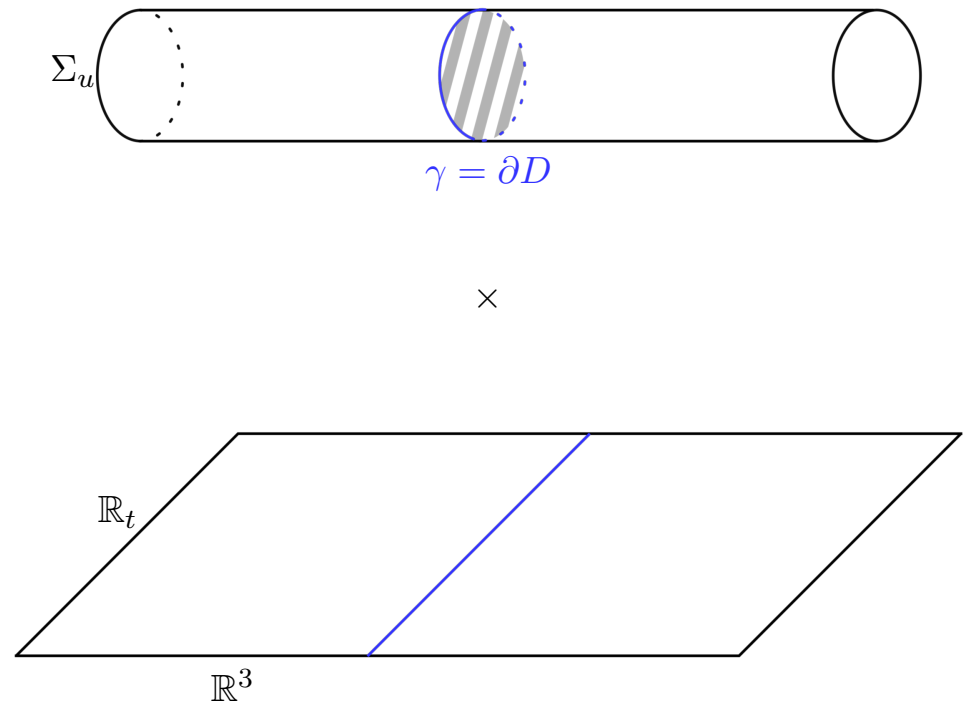

Figure 28. A local picture of an M5-brane wrapping the product of the Seiberg-Witten curve $\Sigma$ with $\mathbb{R}^{3,1}=\mathbb{R}^{3} \times \mathbb{R}_{t}$, with an M2-brane wrapping the product of a two-cycle $D$ with the wordline $\mathbb{R}_{t}$ of a BPS particle in $\mathbb{R}^{3,1}$.

Such a BPS particle in four dimensions can be regarded as a BPS string in six dimensions [90]. It can be embedded in M-theory as an M2-brane wrapping the product of a compact two-cycle $D$ in $T^{*} C$, which ends on $\Sigma$, with its worldline in $\mathbb{R}^{3,1}$ (see figure 28). Its electromagnetic charge $\gamma$ is encoded in the boundary $[\partial D]=\gamma$, while the BPS condition translates into a calibration condition on the two-cycle $D: D$ should be a holomorphic Lagrangian submanifold of $T^{*} C$ with boundary $[\partial D]=\gamma$. In the simplest case, $D$ is topologically a disc, and its projection to the ultraviolet curve $C$ is an interval $I$ bounded on either side by a branch point of type $(i j)$ of the covering $\Sigma \rightarrow C$ (see figure 29). Let $\lambda_{i}$ be the restriction of the Seiberg-Witten differential to the $i$-th sheet; the tension of an (ij)-string is given by $\lambda_{i}-\lambda_{j}$. Then the central charge of the corresponding BPS particle is given by

$$
Z_{i j}=\oint_{\gamma} \lambda=\int_{I}\left(\lambda_{i}-\lambda_{j}\right)
$$

while its mass is

$$
M_{i j}=\int_{I}\left|\lambda_{i}-\lambda_{j}\right|
$$

The BPS condition $M_{i j}=\left|Z_{i j}\right|$ is thus satisfied if and only if the absolute value of the integral of the one-form $\lambda_{i}-\lambda_{j}$ is the same as the integral of its absolute value, which is true if and only if

$$
\mathrm{e}^{-\mathrm{i} \vartheta}\left(\lambda_{i}-\lambda_{j}\right)(v) \in \mathbb{R}^{\times}
$$

for any tangent vector $v$ along the projection of $\gamma$ to $C$, where $\vartheta=\arg \left(Z_{i j}\right)$. (This also specifies which supercharges are preserved by this BPS state.) Note that (3.28) is exactly 


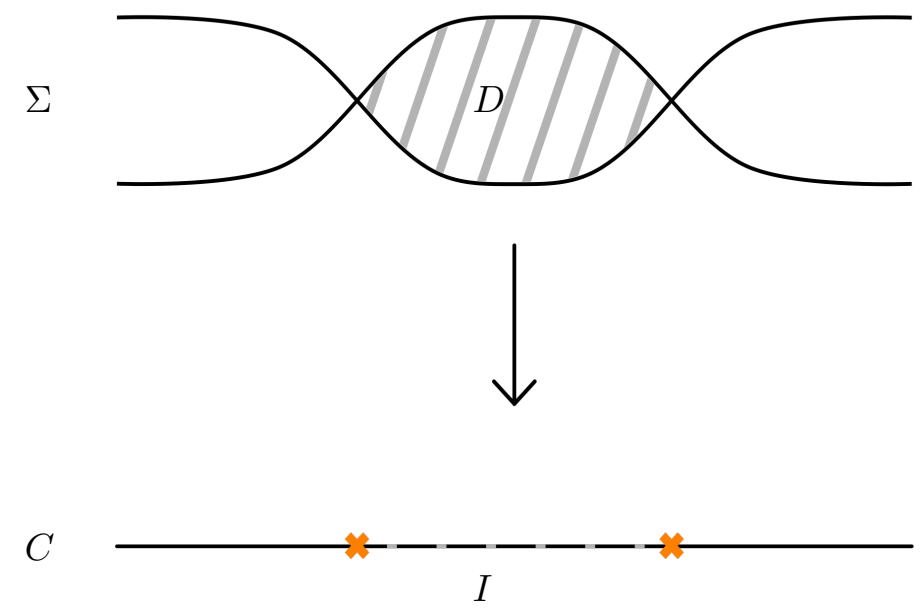

Figure 29. A disc $D$ bounded by $\Sigma$ which projects down to an interval $I$ on $C$.

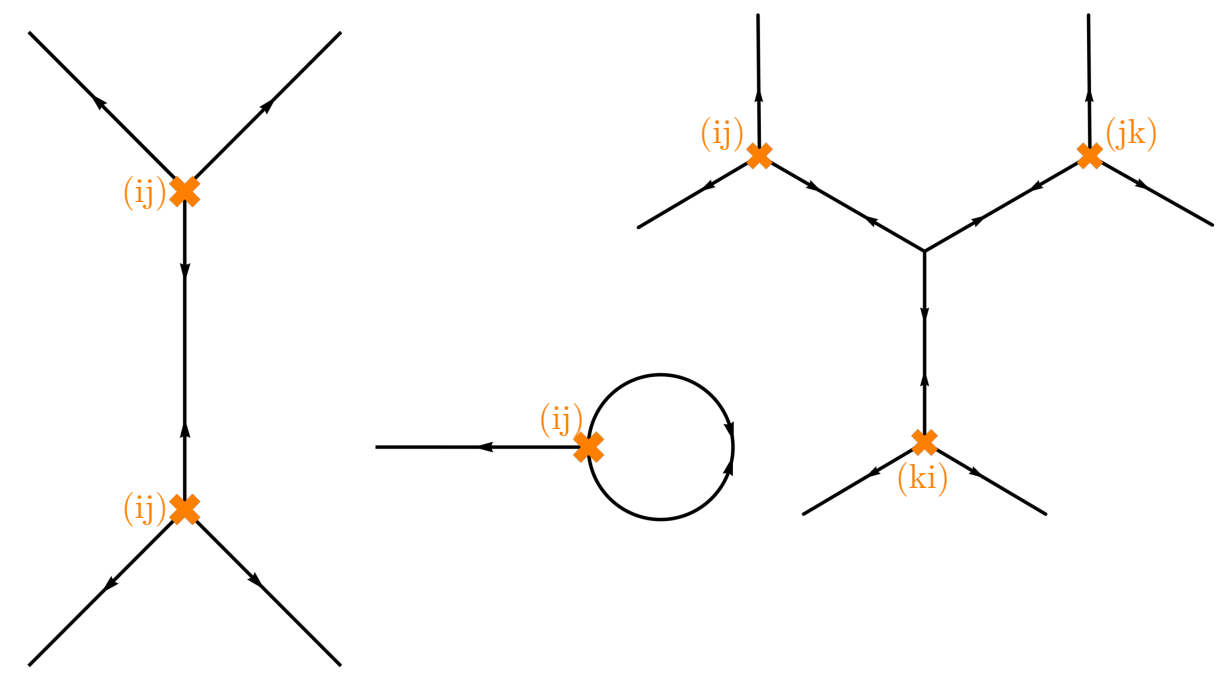

Figure 30. Three examples of finite webs. For class $\mathcal{S}$ theories with $\mathrm{SU}(2)$ gauge group, the first two webs are the only possibilities, but for $\mathrm{SU}(3)$ and higher rank gauge groups, more complicated webs can form, such as the third web shown. We mark the branch points with pairs of indices $i j$ that correspond to the sheets $\Sigma_{i}, \Sigma_{j}$ that come together there, as well as the trajectories that emerge from them, which are of type $(i j)$ or $(j i)$.

the condition for a trajectory of type $(i j)$. In other words, we can identify such a BPS particle of charge $\gamma$ with a saddle of the spectral network $\mathscr{W}(u, \vartheta)$ on $C$ that lifts to $\gamma$ on $\Sigma$.

More generally, any BPS particle in the vacuum $u \in \mathscr{B}$ with central charge $Z=\mathrm{e}^{\mathrm{i} \vartheta} M$ is encoded in the spectral network $\mathscr{W}(u, \vartheta)$ as a finite web [58], a web consisting of a finite number of saddles that have both ends on branch points or junctions (see figure 30 for some examples). If we vary $\vartheta$ and systematically scan for such finite webs, we uncover the spectrum of BPS particles of the class $\mathcal{S}$ theory $\mathrm{T}_{K}[C, \mathcal{D}]$ in the vacuum $u$. The rays in 
the complex $Z$-plane for which there exists a BPS particle in the vacuum $u$ are sometimes called 'BPS rays'. If we vary the point $u$ on the Coulomb branch, the central charges $Z_{\gamma}$ change and thus the BPS rays move in the $Z$-plane.

There might be real codimension one walls in the Coulomb branch $\mathscr{B}$ where the phases of some of the BPS particles coincide. Such walls are called 'walls of marginal stability'. These are the only places where BPS bound states can form or decay. The walls of marginal stability thus divide the Coulomb branch into different regions where there may be a different spectrum of BPS states.

Example 14. Recall example 1 with $C=\mathbb{C}$ and the quadratic differential

$$
p_{2}(z)=\left(z^{2}+m\right) \mathrm{d} z \otimes \mathrm{d} z .
$$

The corresponding four-dimensional $\mathcal{N}=2$ field theory is the simplest example of an Argyres-Douglas theory [12], which is the theory of a single hypermultiplet of mass $m$. It is sometimes called the $\mathrm{AD}_{2}$ theory [59].

The Coulomb branch $\mathscr{B}$ of the $\mathrm{AD}_{2}$ theory is a single point, corresponding to the differential $p_{2}(z)$ with fixed parameter $m$ (the mass of the hypermultiplet), so this theory contains a unique vacuum. Its Seiberg-Witten geometry is illustrated in figure 31; the charge lattice $\Gamma=H_{1}(\Sigma, \mathbb{Z}) \cong \mathbb{Z}$ is generated by the one-cycle $\gamma$.

The spectral networks for this theory were discussed in example 1: if we vary $\vartheta$ from 0 to $\pi$ a saddle appears at $\vartheta=\vartheta_{\mathrm{c}}=\pi / 2$, see figure 3 . The corresponding BPS particle is of course the BPS hypermultiplet of mass $m$. When $m=0$ the two branch points collide, the one-cycle $\gamma$ collapses, and the hypermultiplet becomes massless.

Example 15. Let us now determine the BPS spectrum of the pure SU(2) gauge theory, which we introduced in section 3.1, using spectral networks. The Seiberg-Witten geometry is determined by the quadratic differential

$$
p_{2}(z)=\left(\frac{\Lambda^{2}}{z^{3}}-\frac{2 u}{z^{2}}+\frac{\Lambda^{2}}{z}\right) \mathrm{d} z \otimes \mathrm{d} z,
$$

where $\Lambda$ is the ultraviolet scale and $u$ parametrizes the Coulomb branch $\mathscr{B}$. This differential has irregular (or wild) singularities (of order three) at $z=0$ and $z=\infty$, and two zeroes at

$$
z_{ \pm}=-\frac{u}{\Lambda^{2}} \pm \sqrt{\left(\frac{u}{\Lambda^{2}}\right)^{2}-1} .
$$

The Seiberg-Witten curve $\Sigma$ is defined as $w^{2}=p_{2}(z)$. As we saw in section 3.1, this is topologically a torus with two double punctures, see figure 20 .

Note that there are two special points on the Coulomb branch: at $u= \pm \Lambda^{2}$ both zeroes of $p_{2}(z)$ collide. These are the two quantum singularities of $\mathscr{B}$, where the Seiberg-Witten curve $\Sigma$ degenerates and some one-cycle $\gamma$ vanishes (see figure 32 ). We now know that this signals the presence of massless BPS particles of electromagnetic charge $\gamma$.

Indeed, this can be verified by examining the spectral networks $\mathscr{W}(u, \vartheta)$ close to the singularities. It actually turns out that the $u$-plane is divided into two regions in which 


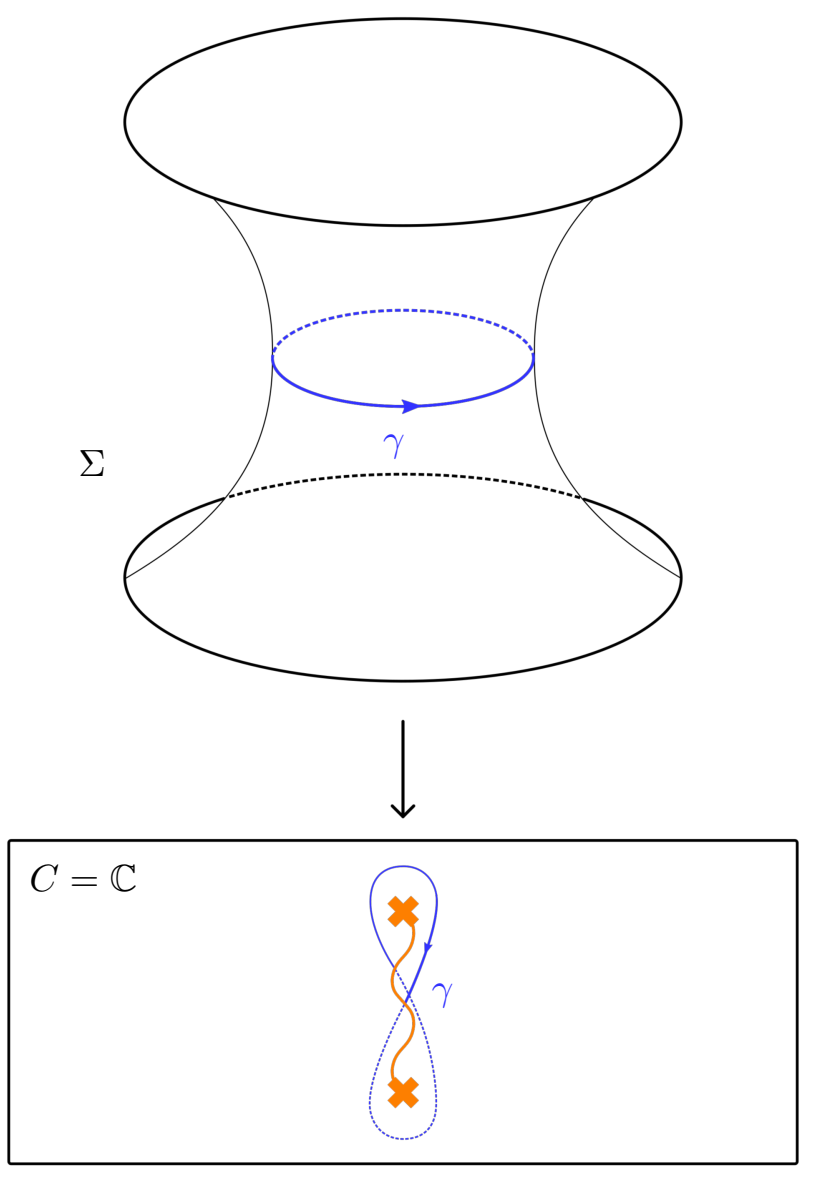

Figure 31. Seiberg-Witten geometry of the $\mathrm{AD}_{2}$ theory.

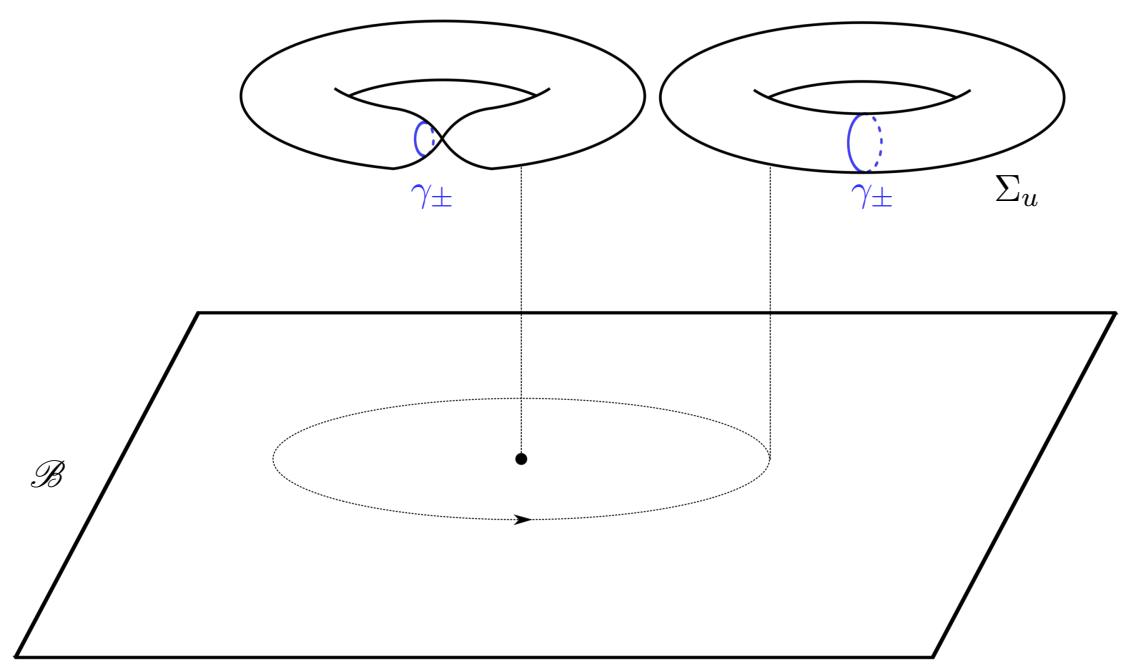

Figure 32. At the singularities $u= \pm \Lambda^{2}$ the Seiberg-Witten curve $\Sigma_{u}$ degenerates because a one-cycle $\gamma_{ \pm}$vanishes. This leads to an $\operatorname{SL}(2, \mathbb{Z})$ monodromy in the charge lattice $\Gamma$ around these two points. 


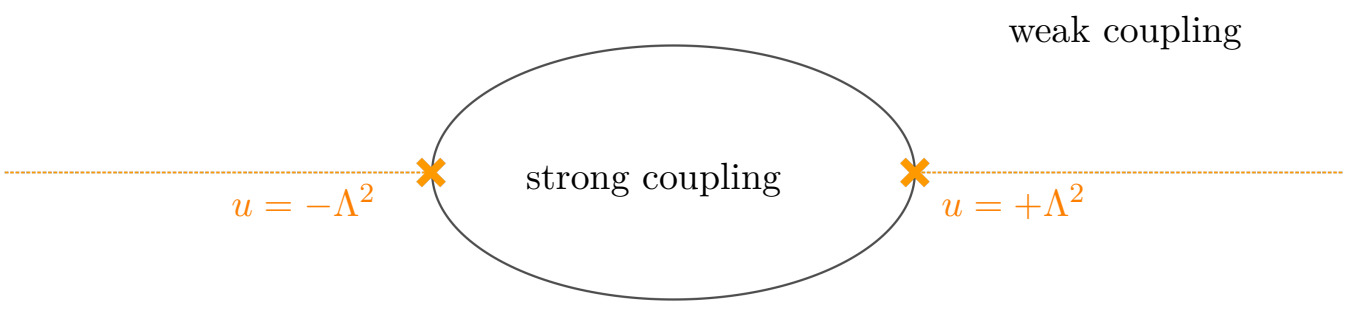

Figure 33. Coulomb branch, or $u$-plane, for the pure $\mathrm{SU}(2)$ theory. The wall of marginal stability, shown in black, separates a strongly coupled region near $u=0$ from a weakly coupled region near $u=\infty$. Since there is an $\operatorname{SL}(2, \mathbb{Z})$ monodromy around the singularities at $u= \pm \Lambda^{2}$, we have chosen the cut, shown in orange, to trivialize the corresponding local system.

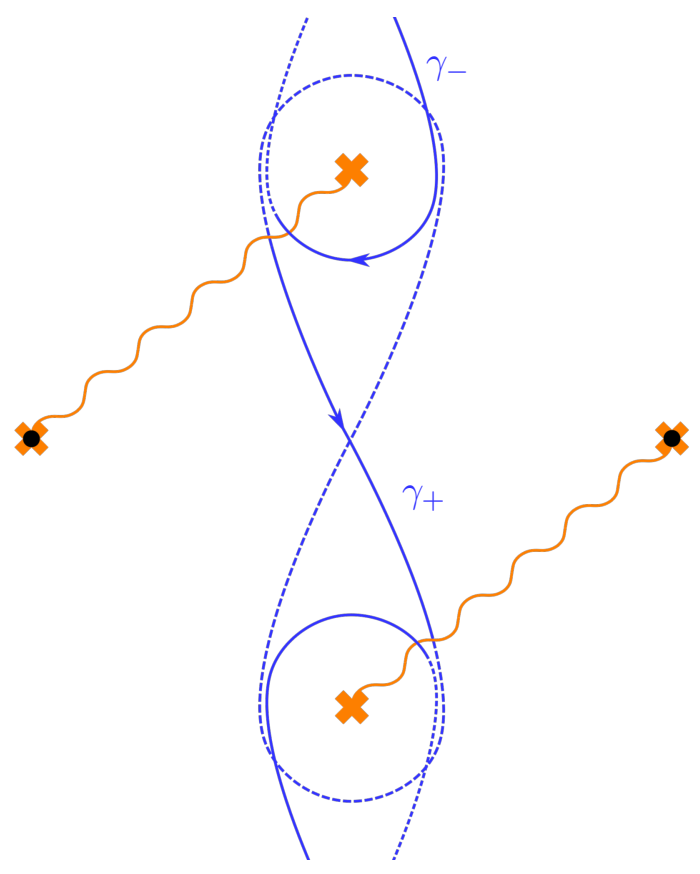

Figure 34. Choice of a basis of one-cycles $\gamma_{ \pm}$of $\Gamma$ drawn on the ultraviolet curve $C$, where we mapped $z=\infty$ to finite distance.

the networks $\mathscr{W}(u, \vartheta)$ behave very differently. These two regions are known as the strong coupling region (for small $u$ ) and the weak coupling region (for large $u$ ). They are divided by a wall of marginal stability, which is defined by the equation $\operatorname{Im}\left(a_{\mathrm{D}} / a\right)=0$ and can be approximated by an ellipse passing through the singularities (see figure 33). We choose cuts for the $\mathrm{SL}(2, \mathbb{Z})$ monodromy as in figure 33 , and trivialize the corresponding local system by choosing a basis of the charge lattice $\Gamma$ as in figure 34 .

Let us consider the family of spectral networks $\mathscr{W}(u, \vartheta)$ for varying phase $\vartheta$ at a point $u$ in the strongly coupled region first. In figure 35 we illustrate the topology changes that occur as $\vartheta$ is varied from $-\frac{\pi}{4}$ to $\frac{3 \pi}{4}$ at $u=0$. (The picture would be similar at any other point in the strongly coupled region.) In this family we encounter two saddles, at $\vartheta=0$ 

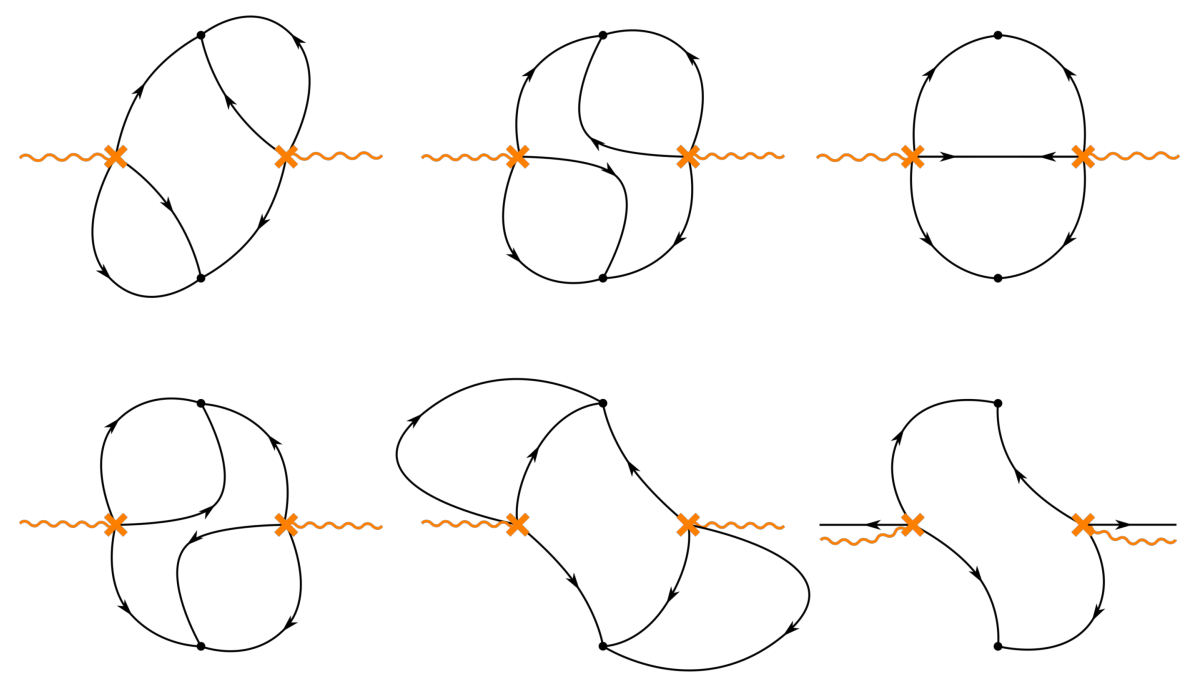

Figure 35. The spectral networks for the pure $\mathrm{SU}(2)$ theory as $\vartheta$ is varied over two flips. The networks are rotated through $-90^{\circ}$.

and $\vartheta=\frac{\pi}{2}$. The first saddle corresponds to a hypermultiplet of electromagnetic charge $\gamma_{+}$ and the second saddle to a hypermultiplet of electromagnetic charge $\gamma_{-}$. If we continue varying $\vartheta$ from $\frac{3 \pi}{4}$ to $\frac{7 \pi}{4}$, we come across two saddles corresponding to their antiparticles. The BPS spectrum in the strongly coupled region thus consists of two hypermultiplets, traditionally called the monopole and the dyon, and their charge conjugates.

The story in the weakly coupled region is slightly more complicated. Fortunately we have already studied the relevant networks in example 3 . Topology changes occur when $\vartheta$ is tuned to a critical phase $\vartheta_{\mathrm{c}}$ (as well as $\pi+\vartheta_{\mathrm{c}}$ ), see figure 6 . As $\vartheta$ increases to $\vartheta_{\mathrm{c}}$ we encounter an infinite number of flips, which correspond to an infinite tower of hypermultiplets with charges $(n+1) \gamma_{+}+n \gamma_{-}$for $n \in \mathbb{Z}_{\geq 0}$. The juggle at $\vartheta=\vartheta_{\text {c }}$ encodes a vector multiplet of charge $\gamma_{+}+\gamma_{-}$, and the infinite tower of flips as $\vartheta$ decreases to $\vartheta_{\mathrm{c}}$ correspond to another infinite number of hypermultiplets with charges $n \gamma_{+}+(n+1) \gamma_{-}$. At the phase $\vartheta=\pi+\vartheta_{\mathrm{c}}$ we find all of their charge conjugates.

Evidently, the spectra on either side of the wall of marginal stability are not the same, and some BPS states have decayed. This may be captured by the formula

$$
K_{\gamma_{+}} K_{\gamma_{-}}=K_{\gamma_{-}} K_{\gamma_{+}+2 \gamma_{-}} K_{2 \gamma_{+}+3 \gamma_{-}} \cdots K_{\gamma_{+}+\gamma_{-}}^{2} \cdots K_{3 \gamma_{+}+2 \gamma_{-}} K_{2 \gamma_{+}+\gamma_{-}} K_{\gamma_{+}},
$$

where $K_{\gamma}$ is the transformation

$$
K_{\gamma}: \mathcal{X}_{\gamma^{\prime}} \longmapsto \mathcal{X}_{\gamma^{\prime}}\left(1-(-1)^{\left\langle\gamma^{\prime}, \gamma\right\rangle} \mathcal{X}_{\gamma}\right)^{\left\langle\gamma^{\prime}, \gamma\right\rangle}
$$

of the spectral coordinates $\mathcal{X}_{\gamma^{\prime}}$ under a flip [59, section 7.6]. This is the famous KontsevichSoibelman wall-crossing formula [94]. For a good introduction to wall-crossing in $\mathcal{N}=2$ field theories, we recommend [105].

By now there is rich body of work on BPS states and wall-crossing in $\mathcal{N}=2$ theories, from many points of view. Of special note is the BPS quiver method, which is particularly 
powerful when there is a region (or chamber) in the Coulomb branch with a finite BPS spectrum. This led to the determination of the BPS spectra of all "complete" $\mathcal{N}=2$ theories [8].

The beautifully geometric spectral network method can be applied to any $\mathcal{N}=2$ theory of class $\mathcal{S}$ at any point $u \in \mathscr{B}$ of its Coulomb branch. It turns out to be especially valuable for conformal $\mathcal{N}=2$ theories with a single infinite BPS chamber. Examples include the intrinsically strongly coupled $E_{6}$ and higher rank Minahan-Nemeschansky theories discussed in section 3.2 , whose spectra are determined in $[69,76]$, where higher rank Fenchel-Nielsen networks play a crucial role. In these examples one finds not only saddles corresponding to hypermultiplets or closed loops corresponding to vector multiplets, but also more complicated topologies arise, such as the finite webs of figure 30 . The method of spectral networks can also be extended to compute the spin content of BPS states, with the spin identified with the writhe of paths on the Seiberg-Witten curve $\Sigma$ [62], which is related to a quantization of the moduli space of flat abelian connections on $\Sigma$. Further developments and applications of the spectral network method can be found in e.g. [61, 96, 97], while the connection between the techniques based on spectral networks and BPS quivers is discussed in [52].

\section{Twisted superpotentials, Hitchin systems and opers}

In this section we discuss a certain physical quantity, called the effective twisted superpotential $\widetilde{\mathcal{W}}^{\text {eff }}$ of an $\mathcal{N}=2$ field theory $\mathrm{T}_{K}[C, \mathcal{D}]$ of class $\mathcal{S}$ in the $\frac{1}{2} \Omega$-background; this is introduced in section 4.1. Interestingly, in [114] it was conjectured that $\widetilde{\mathcal{W}}^{\text {eff }}$ has a concrete geometric meaning, which may be extended to any $\mathcal{N}=2$ theory of class $\mathcal{S}$, in the Hitchin moduli space. This conjecture will be formulated in section 5 , while in the remainder of this section we explain the various geometric ingredients: the Hitchin integrable system in section 4.2, and its Lagrangian subspace of oper connections in section 4.3. We give an explicit description of the relevant oper connections in our main examples, and relate the abelianization and spectral coordinates associated to these connections with exact WKB methods in section 4.4 .

\subsection{Effective twisted superpotentials}

For any $\mathcal{N}=2$ theory $\mathrm{T}$, the low energy dynamics on its Coulomb branch $\mathscr{B}$ can be described in terms of its holomorphic prepotential $\mathcal{F}_{0}(a ; m ; q)$. This is a multi-valued analytic function depending on the Coulomb moduli $a$, the mass parameters $m$, and the exponentiated ultraviolet gauge couplings $q$. We have seen that in terms of Seiberg-Witten geometry it can be obtained from period integrals

$$
a_{i}=\oint_{A^{i}} \lambda \quad \text { and } \quad a_{\mathrm{D}}^{i}=\oint_{B_{i}} \lambda
$$

on the Seiberg-Witten curve $(\Sigma, \lambda)$ via the relation

$$
a_{\mathrm{D}}^{i}=\frac{\partial \mathcal{F}_{0}(a ; m ; q)}{\partial a_{i}} .
$$


Nekrasov computed $\mathcal{F}_{0}$ from first principles for $\mathcal{N}=2$ theories $\mathrm{T}$ with a Lagrangian formulation (that is, in terms of gauge fields possibly coupled to matter fields) [111] by considering a deformation of the theory T labeled by two complex parameters $\epsilon_{1}$ and $\epsilon_{2}$. This deformation is called the $\Omega$-background, ${ }^{23}$ where the two parameters $\epsilon_{1}$ and $\epsilon_{2}$, both with dimensions of mass, correspond to two isometries rotating two-planes in $\mathbb{R}^{4}$ according to the splitting

$$
\mathbb{R}^{4}=\mathbb{R}_{\epsilon_{1}}^{2} \times \mathbb{R}_{\epsilon_{2}}^{2} .
$$

The low energy dynamics of the resulting theory $\mathrm{T}_{\epsilon_{1}, \epsilon_{2}}$ is described by a prepotential $\mathcal{F}\left(a ; m ; q ; \epsilon_{1}, \epsilon_{2}\right)$ which is a deformation of $\mathcal{F}_{0}(a ; m ; q)$ in the sense that its limit, as $\epsilon_{1}$ and $\epsilon_{2}$ are sent to zero, is the prepotential $\mathcal{F}_{0}(a ; m ; q)$. More precisely, in an expansion in $\epsilon_{1}, \epsilon_{2}$ near zero, the function $\epsilon_{1} \epsilon_{2} \mathcal{F}\left(a ; m ; q ; \epsilon_{1}, \epsilon_{2}\right)$ is analytic and we write

$$
\mathcal{F}\left(a ; m ; q ; \epsilon_{1}, \epsilon_{2}\right)=\frac{1}{\epsilon_{1} \epsilon_{2}} \mathcal{F}_{0}(a ; m ; q)+\text { terms regular in } \epsilon_{1}, \epsilon_{2} .
$$

The gauge theory partition function $Z^{\mathrm{Nek}}\left(a ; m ; q ; \epsilon_{1}, \epsilon_{2}\right)=\exp \mathcal{F}\left(a ; m ; q ; \epsilon_{1}, \epsilon_{2}\right)$ of the theory $\mathrm{T}_{\epsilon_{1}, \epsilon_{2}}$ is sometimes called the Nekrasov partition function. After twisting the theory with a Donaldson twist, the Nekrasov partition function may be computed as an equivariant integral over the moduli space of instantons on $\mathbb{R}^{4}$. The resulting prepotential $\mathcal{F}$ can be decomposed into a classical term plus contributions from one-loop and instanton effects as

$$
\mathcal{F}=\mathcal{F}_{\text {cl }}+\mathcal{F}_{1 \text {-loop }}+\mathcal{F}_{\text {inst }}
$$

The classical contribution is

$$
\mathcal{F}_{\mathrm{cl}}=\frac{1}{\epsilon_{1} \epsilon_{2}} \log q \sum_{i=1}^{r} a_{i}^{2}
$$

where $r$ is the rank of the gauge algebra $\mathfrak{g}$. The one-loop contribution is independent of $q$ and may be computed as a product of determinants of differential operators. The result depends on the particle multiplets involved; for example, in the case of a hypermultiplet of mass $m$ one finds

$$
\mathcal{F}_{1 \text {-loop }}=\frac{1}{\epsilon_{1} \epsilon_{2}}\left(\frac{1}{2} m^{2} \log m-\frac{3}{4} m^{2}\right)+\text { terms regular in } \epsilon_{1}, \epsilon_{2} .
$$

Lastly, the instanton contribution may be written as a sum over Young diagrams (for $\mathfrak{g}=\mathfrak{s u}(K))$, and it has a power series expansion in the exponentiated gauge couplings $q=\mathrm{e}^{2 \pi \mathrm{i} \tau_{\mathrm{UV}}}$ of the form

$$
\mathcal{F}_{\text {inst }}=\sum_{k=1}^{\infty} c_{k} q^{k}
$$

More about Nekrasov partition functions may be found in [113, 115, 128, 130, 133].

In the following we will be interested in the special case where $\epsilon_{2}=0$ while $\epsilon_{1}=\epsilon$ is kept finite, which is also known as the Nekrasov-Shatashvili limit or the $\frac{1}{2} \Omega$-background. The resulting theory $\mathrm{T}_{\epsilon}=\mathrm{T}_{\epsilon, 0}$ preserves a two-dimensional $\mathcal{N}=(2,2)$ supersymmetry in the

\footnotetext{
${ }^{23}$ The precise construction of this background (starting from six dimensions) is given for instance in [114, section 1.4].
} 
$\mathbb{R}_{34}^{2}$-plane. ${ }^{24}$ The physics of $\mathbf{T}_{\epsilon}$ has been discussed extensively in the work of Nekrasov and Shatashvili, see in particular [115]. It is proposed that in the infrared limit, at energies $E \ll$ $|\epsilon|$, the theory $\boldsymbol{T}_{\epsilon}$ is described by $r$ abelian vector multiplets, coupled to an effective twisted superpotential $\widetilde{\mathcal{W}}^{\text {eff }}(\boldsymbol{\Sigma} ; m ; q ; \epsilon)$ built from the twisted chiral superfields $\boldsymbol{\Sigma}$ in the abelian vector multiplets. Moreover, if one restricts $\widetilde{\mathcal{W}}^{\text {eff }}(\boldsymbol{\Sigma} ; m ; q ; \epsilon)$ to the lowest components $\sigma_{i}=a_{i}$ of $\boldsymbol{\Sigma}$, it was proposed that

$$
\widetilde{\mathcal{W}}^{\mathrm{eff}}(a ; m ; q ; \epsilon)=\lim _{\epsilon_{2} \rightarrow 0} \epsilon_{2} \mathcal{F}\left(a ; m ; q ; \epsilon_{1}=\epsilon, \epsilon_{2}\right)
$$

In particular, the theory $\mathrm{T}_{\epsilon}$ has a discrete set of vacua determined as solutions to the quantization condition

$$
\exp \left(\frac{\partial \widetilde{\mathcal{W}}^{\mathrm{eff}}(a ; m ; q ; \epsilon)}{\partial a_{i}}\right)=1
$$

Example 16. The effective twisted superpotential $\widetilde{\mathcal{W}}^{\text {eff }}$ of the simplest Argyres-Douglas theory, the $\mathrm{AD}_{2}$ theory from example 14 , in the $\frac{1}{2} \Omega$-background has only a one-loop contribution from the free hypermultiplet of mass $m$. Hence ${ }^{25}$

$$
\widetilde{\mathcal{W}}^{\mathrm{eff}}(m ; \epsilon)=\frac{\epsilon}{2} \Upsilon\left(\frac{1}{2}+\frac{m}{2 \epsilon}\right)
$$

where

$$
\Upsilon(x)=\int_{\frac{1}{2}}^{x} \log \frac{\Gamma\left(x^{\prime}\right)}{\Gamma\left(1-x^{\prime}\right)} \mathrm{d} x^{\prime}
$$

Example 17. The effective twisted superpotential $\widetilde{\mathcal{W}}^{\text {eff }}$ of the pure $\mathrm{SU}(2)$ theory, discussed in section 3.1 , in the $\frac{1}{2} \Omega$-background has a classical, a one-loop and an instanton contribution $^{26}$ as a series expansion in powers of the ultraviolet scale parameter $\Lambda$ (which

${ }^{24}$ This can be shown by decomposing the supercharges of the original theory $\mathrm{T}$, which transform in the representation $\left(\mathbf{2}^{+} \oplus \mathbf{2}^{-}, \mathbf{2}\right)$ of $\mathfrak{s o}(4, \mathbb{C}) \times \mathfrak{s l}(2, \mathbb{C})_{R}$, under the subalgebra $\mathfrak{g l}(1, \mathbb{C}) \times \mathfrak{g l}(1, \mathbb{C}) \times \mathfrak{g l}(1, \mathbb{C})_{R}$ of complexified rotations in the 12-plane and the 34-plane, and R-symmetry rotations in the 12-plane; this decomposes into eight weight spaces with weights $( \pm 1, \pm 1, \pm 1)$. Four of the resulting charges are invariant under the generator $\epsilon\left(J^{12}+J_{R}^{12}\right)$ of the $\frac{1}{2} \Omega$-background, where $J^{12}$ is a generator of rotations in $\mathbb{R}_{12}^{2}$ and $J_{R}^{12}$ is an R-symmetry generator, and these generate $\mathcal{N}=(2,2)$ supersymmetry in the 34-plane.

${ }^{25}$ The one-loop contribution $\exp \widetilde{\mathcal{W}}_{1-\text { loop }}^{\text {eff }}$ may be computed as a product of determinants of differential operators. There is a certain freedom in its definition due to ambiguities in the regularization of divergences, which implies that it is only determined up to a phase [121, 137]. For a distinguished choice of phase, $\exp \widetilde{\mathcal{W}}^{\text {eff }}$ can be identified with the square root of the product of two Liouville three-point functions in the Nekrasov-Shatashvili (or $c \rightarrow \infty$ ) limit. The one-loop contributions that we use are computed in this "Liouville scheme".

${ }^{26}$ To be precise, the Nekrasov partition function computes the instanton contributions to the U(2) gauge theory. It is possible to extract the "spurious" U(1) contribution, which does not depend on the Coulomb parameter $a$, by comparing it to a dual Liouville conformal block [5], or alternatively by computing the $\operatorname{Sp}(1)$ Nekrasov partition function. The latter is related to the former by a change of ultraviolet regularization scheme [72]. 
replaces the instanton coupling $q$ in asymptotically free theories). Explicitly

$$
\begin{aligned}
& \widetilde{\mathcal{W}}_{\mathrm{cl}}^{\text {eff }}(a ; \Lambda ; \epsilon)=\frac{a^{2}}{\epsilon} \log \left(\frac{\Lambda}{\epsilon}\right), \\
& \widetilde{\mathcal{W}}_{1-\text { loop }}^{\text {eff }}(a ; \epsilon)=-\frac{\epsilon}{2} \Upsilon\left(-\frac{a}{\epsilon}\right)-\frac{\epsilon}{2} \Upsilon\left(\frac{a}{\epsilon}\right), \\
& \widetilde{\mathcal{W}}_{\text {inst }}^{\text {eff }}(a ; \Lambda ; \epsilon)=\frac{2}{\epsilon\left(a^{2}-\epsilon^{2}\right)} \Lambda^{4}+\frac{5 a^{2}+7 \epsilon^{2}}{\epsilon\left(a^{2}-4 \epsilon^{2}\right)\left(a^{2}-\epsilon^{2}\right)^{3}} \Lambda^{8}+O\left(\Lambda^{12}\right) .
\end{aligned}
$$

As shown by [102], the parameter $a$ in (4.13) is not proportional to the classical period $\Pi_{A}^{(0)}=\oint_{A} \lambda$, but instead receives $\epsilon$-corrections. As we will see later, the $\epsilon$-expansion for $a$ may be obtained by computing a quantum period $\Pi_{A}(\epsilon)$ which is defined in (4.81), while the quantum period $\Pi_{B}(\epsilon)$ computes the $\epsilon$-expansion for $\widetilde{\mathcal{W}}^{\text {eff }}{ }^{27}$

Although the Nekrasov partition function can only be computed from first principles for $\mathcal{N}=2$ field theories with a Lagrangian description, one expects that a similar object can be defined for any $\mathcal{N}=2$ theory and, in particular, for any $\mathcal{N}=2$ theory of class $\mathcal{S}$. For attempts to compute this object from either string theory, five dimensions or conformal field theory, see e.g. [2, 3, 17, 37, 78, 103, 104].

Example 18. The effective twisted superpotential $\widetilde{\mathcal{W}}^{\text {eff }}$ of the intrinsically strongly coupled "non-Lagrangian" $E_{6}$ Minahan-Nemeschansky theory, discussed in section 3.2, in the $\frac{1}{2} \Omega$ background is (to our knowledge) so far unknown in explicit form. We will return to this open problem in section 5.5 below.

\subsection{Hitchin systems}

Let us now introduce the Hitchin moduli space [71]. We start by explaining how fourdimensional $\mathcal{N}=2$ field theories are related to integrable systems (more details can be found in the review [109]). Of particular relevance is the notion of a (classical) algebraic integrable system. This is a triple $(\mathscr{I}, \Omega, \mathscr{B})$, where $(\mathscr{I}, \Omega)$ is a complex $2 r$-dimensional holomorphic symplectic manifold and $\mathscr{B}$ is a complex $r$-dimensional manifold, together with a holomorphic fibration $\pi: \mathscr{I} \rightarrow \mathscr{B}$ whose generic fibers $\mathscr{I}_{u}=\pi^{-1}(u)$ are polarized abelian varieties of complex dimension $r$.

Recall that the low energy description of any $\mathcal{N}=2$ theory is encoded in the auxiliary Seiberg-Witten geometry $(\Sigma, \lambda)$. Soon after the discoveries of Seiberg and Witten, it was realized that this description can be formulated mathematically as an algebraic integrable system of dimension $r$ given by the rank of the gauge algebra [44]. Its base manifold $\mathscr{B}$ is the Coulomb branch of the $\mathcal{N}=2$ theory and its fibers are the complex tori $\mathbb{C}^{r} / \Gamma_{u}$, where $\Gamma_{u}=H_{1}\left(\bar{\Sigma}_{u}, \mathbb{Z}\right)$ is the charge lattice at the vacuum $u$, polarized by the choice of $A$-cycles and $B$-cycles on $\bar{\Sigma}_{u}$ (the electric-magnetic splitting). Even more concretely, it was found for instance that the pure $\mathrm{SU}(K)$ gauge theory corresponds to the periodic $A_{K-1}$ Toda chain [64].

\footnotetext{
${ }^{27}$ More precisely, if we define the $\epsilon$-expansion for $a$ by $\frac{\pi \mathrm{i}}{\epsilon} a=\Pi_{A}(\epsilon)$, then the $\epsilon$-expansion for $\widetilde{\mathcal{W}}^{\text {eff }}(a ; \Lambda ; \epsilon)$ is obtained from the equation $\Pi_{B}(\epsilon)=2 \partial_{a} \widetilde{\mathcal{W}}^{\text {eff }}(a ; \Lambda ; \epsilon)$.
} 
However, so far only the base $\mathscr{B}$ of the algebraic integrable system has a description in terms of the fields of the $\mathcal{N}=2$ theory. The complete picture emerges if we compactify the four-dimensional $\mathcal{N}=2$ theory on a circe $S^{1}$ of radius $R$ [125]. At low energies $E \ll R^{-1}$, the resulting three-dimensional field theory $\mathrm{T}[R]$ can be described in terms of $r$ complex scalars and $r$ abelian gauge fields in three dimensions, together with $2 r$ periodic real scalars. The latter are the holonomies of the $r$ abelian and $r$ dualized abelian gauge fields in four dimensions around the compactified direction $S^{1}$. The complex scalars parameterize a sigma-model into $\mathscr{B}$, and we can think of the $2 r$ periodic real scalars as giving a map into a complex $r$-dimensional torus. Taking into account electric-magnetic duality transformations, we may conclude that $\mathrm{T}[R]$ is a three-dimensional sigma-model whose target $\mathscr{M}$ is diffeomorphic to the total space $\mathscr{I}$ of the algebraic integrable system.

Invariance under three-dimensional $\mathcal{N}=4$ supersymmetry demands that the target space $\mathscr{M}$ of the sigma-model is then a hyperkähler manifold. ${ }^{28}$ This is a kähler manifold which has a triple of complex structures $(I, J, K)$ with respect to each of which its metric $g$ is kähler. The complex structures $(I, J, K)$ satisfy the quaternion algebra

$$
I^{2}=J^{2}=K^{2}=-1=I J K .
$$

With the kähler forms $\omega_{I}, \omega_{J}$ and $\omega_{K}$ of $\mathscr{M}$ one can construct holomorphic symplectic forms $\Omega_{I}, \Omega_{J}$ and $\Omega_{K}$ given by

$$
\Omega_{I}(\cdot, \cdot)=g((J+\mathrm{i} K) \cdot, \cdot)=\omega_{J}(\cdot, \cdot)+\mathrm{i} \omega_{K}(\cdot, \cdot),
$$

and its cyclic permutations in $(I, J, K)$. One of these complex structures, say $I$, is distinguished since the target space $\mathscr{M}^{I}$, regarded as a holomorphic symplectic manifold with respect to this complex structure, is biholomorphic to the integrable system $(\mathscr{I}, \Omega)$. For later use we note that, given a hyperkähler structure, we can use the twistor prescription to construct a family of complex structures $J_{r}$ by combining

$$
J_{r}=r_{1} I+r_{2} J+r_{3} K \quad \text { for } \quad \boldsymbol{r} \in S^{2} \hookrightarrow \mathbb{R}^{3} .
$$

It is customary to identify $S^{2} \cong \mathbb{P}^{1}$ and parametrize it by complex numbers $\zeta$. The hyperkähler manifold $\mathscr{M}$ is kähler with respect to the family $J_{\zeta}$ and we can define a corresponding family of holomorphic symplectic forms $\Omega_{\zeta}$ parameterized by $\zeta \in \mathbb{P}^{1}$.

For any $\mathcal{N}=2$ theory $\mathrm{T}=\mathrm{T}_{K}[C, \mathcal{D}]$ of class $\mathcal{S}$, the resulting three-dimensional theory $\mathrm{T}[R]$ is a three-dimensional sigma-model into the moduli space $\mathscr{M}_{\mathrm{H}}(R)$ of solutions to the Hitchin equations on the Riemann surface $C[32,59]$. This is the moduli space of solutions $(A, \varphi)$ to the equations

$$
\begin{aligned}
F_{A}+R^{2}[\varphi, \bar{\varphi}] & =0, \\
\bar{\partial}_{A} \varphi & =0, \\
\partial_{A} \bar{\varphi} & =0,
\end{aligned}
$$

\footnotetext{
${ }^{28}$ By compactifying a four-dimensional $\mathcal{N}=2$ theory to three dimensions, the eight real supercharges that make up the $\mathcal{N}=2$ supersymmetry algebra in four dimensions are rearranged into an $\mathcal{N}=4$ supersymmetry algebra in three dimensions, as the respective spin group changes from $\operatorname{SL}(2, \mathbb{C})$ to $\mathrm{SL}(2, \mathbb{R})$. This is easiest to see by taking the compactified direction to be the $x_{2}$-axis. With the standard parametrization of $\operatorname{SL}(2, \mathbb{C})$ matrices in terms of coordinates of $\mathbb{R}^{3,1}$, we then get real instead of complex matrices.
} 
where $A$ is a connection on an $\mathrm{SU}(K)$-bundle $E \rightarrow C$ with curvature $F_{A}$, and $\varphi$ is a Higgs field which is a holomorphic one-form $\varphi \in \Omega^{1,0}(C$, End $E)$ with suitable boundary conditions at the punctures of $C .{ }^{29}$ The holomorphic structure is defined by the twisted Dolbeault differential $\bar{\partial}_{A}$, and $\bar{\varphi}$ denotes the Hermitean conjugate of $\varphi$.

Indeed, the $\mathcal{N}=2$ theory $\mathrm{T}$ can be obtained from the six-dimensional $(2,0)$-theory $\mathfrak{X}[K]$ by twisted compactification on $C$. If we reverse the order of compactifications, by first compactifying on $S^{1}$, the resulting theory in the infrared limit is the five-dimensional supersymmetric Yang-Mills theory with gauge algebra $\mathfrak{s u}(K)$. Compactifying this theory on $C$, with a partial twist on $C$ that changes the internal field into a complex one-form $\varphi$, gives an effective description of the three-dimensional theory $\mathrm{T}[R]$ as an $\mathcal{N}=4$ sigmamodel into the moduli space of vacuum configurations of five-dimensional supersymmetric Yang-Mills theory on $C \times \mathbb{R}^{2,1}$ that are translation invariant in the non-compact directions. These are precisely the solutions to the Hitchin equations. The Hitchin equations can similarly be obtained by dimensional reduction of the self-dual Yang-Mills equations in four dimensions to equations in two dimensions [20, 70].

The Hitchin moduli space $\mathscr{M}_{\mathrm{H}}(R)$ is well-known to be a hyperkähler manifold, with a family of complex structures $J_{\zeta}$ for $\zeta \in \mathbb{P}^{1}$. Its complex geometry depends crucially on whether $\zeta \in\{0, \infty\}$ or $\zeta \in \mathbb{C}^{\times}$.

Let us start with $\zeta=0$, when $\left(\mathscr{M}_{\mathrm{H}}, J_{0}\right)$ is biholomorphic to the moduli space $\mathscr{M}_{\text {Dol }}(C)$ of stable Higgs bundles $\left(V, \varphi, \bar{\partial}_{A}\right)$ on $C$. A Higgs bundle is a holomorphic vector bundle $\left(V, \bar{\partial}_{A}\right)$ of rank $K$ on $C$ together with a Higgs field $\varphi$, which is a holomorphic one-form on $C$ taking values in the endomorphism bundle End $V$. The moduli space $\mathscr{M}_{\mathrm{Dol}}(C)$ has the structure of an algebraic integrable system, where the projection $\pi: \mathscr{M}_{\text {Dol }}(C) \rightarrow \mathscr{B}$ is defined by taking the characteristic polynomial of $\varphi$. Any point $u \in \mathscr{B}$ thus defines an algebraic curve $\Sigma \subset T^{*} C$ as

$$
\operatorname{det}(w-\varphi)=w^{K}-\sum_{k=2}^{K} p_{k} w^{K-k}=0,
$$

which is the same as the spectral curve (3.20). The Coulomb branch $\mathscr{B}$ of any $\mathcal{N}=2$ field theory of class $\mathcal{S}$ may therefore be identified with the space of $K-1$-tuples $\left(p_{2}, \ldots, p_{K}\right)$ of meromorphic $k$-differentials on $C$, as asserted in section 3.2. The differentials $p_{k}$ may be thought of as a maximal set of algebraically independent commuting Hamiltonians of the integrable system. Similarly, at $\zeta=\infty$ the Hitchin moduli space is biholomorphic to $\overline{\mathscr{M}_{\text {Dol }}(C)}$.

For any $\zeta \in \mathbb{C}^{\times}$, it is useful to consider the complex-valued connection

$$
\mathcal{A}=\frac{R}{\zeta} \varphi+A+R \zeta \bar{\varphi} .
$$

Since the Hitchin equations are equivalent to the flatness of $\mathcal{A}$, the Hitchin moduli space $\left(\mathscr{M}_{\mathrm{H}}, J_{\zeta}\right)$ for $\zeta \in \mathbb{C}^{\times}$may be identified with de Rham moduli space $\mathscr{M}_{\text {flat }}(C, \mathrm{SL}(K, \mathbb{C}))$

\footnotetext{
${ }^{29} \mathrm{~A}$ rigorous discussion of these boundary conditions for Hitchin's equations and the construction of their hyperkähler moduli spaces has been given in [92, 108] for regular singularities, and in [21] for irregular singularities. For our purposes the discussion of boundary conditions on flat $\operatorname{SL}(K, \mathbb{C})$ connections in section 2 (and in section 4.4 below) is sufficient.
} 
of flat $\mathrm{SL}(K, \mathbb{C})$ connections on $C$, with natural holomorphic symplectic form $\Omega_{\zeta}$ given by (2.28). These equivalences are made precise by the non-abelian Hodge correspondence, which gives a diffeomorphism between $\mathscr{M}_{\text {Dol }}(C)$ and $\mathscr{M}_{\text {flat }}(C, \operatorname{SL}(K, \mathbb{C}))[45,71,126]$.

The different descriptions of $\left(\mathscr{M}_{\mathrm{H}}, J_{\zeta}\right)$ for $\zeta \in \mathbb{C}$ may be combined into a new notion known as a flat $\lambda$-connection (see e.g. [127]). This is an object $\nabla_{\lambda}$ that interpolates between a Higgs bundle and a flat connection. Instead of the usual Leibniz rule

$$
\nabla(f s)=\mathrm{d} f \otimes s+f \nabla s
$$

obeyed by a connection $\nabla$ acting on the multiplication of a section $s$ by a function $f$, it obeys the more general Leibniz rule

$$
\nabla_{\lambda}(f s)=\lambda \mathrm{d} f \otimes s+f \nabla_{\lambda} s
$$

for $\lambda \in \mathbb{C}$. In the present case, multiplying (4.19) by $\epsilon=\zeta / R$ gives a flat $\epsilon$-connection, which interpolates between a Higgs field $\varphi$ for $\epsilon=0$ and a flat connection for $\epsilon=1{ }^{30}$

\subsection{Opers}

The moduli space of flat $\epsilon$-connections $\mathscr{M}_{\text {flat }}^{\epsilon}(C, \mathrm{SL}(K, \mathbb{C}))$ has, for $\epsilon \neq 0$, a distinguished holomorphic Lagrangian submanifold $\mathscr{L}_{\epsilon}^{\text {oper }} \subset \mathscr{M}_{\text {flat }}^{\epsilon}(C, \mathrm{SL}(K, \mathbb{C}))$, which is known as the brane of $\epsilon$-opers [19]. They feature mathematically in the geometric Langlands program [51] and its gauge theory interpretation [88]. They also play an important role in the non-abelian Hodge correspondence [48, 49], following a conjecture by Gaiotto [55]. This conjecture states that in the "conformal limit" $R, \zeta \rightarrow 0$ with $\zeta / R=\epsilon$ fixed, the $\epsilon$-connection $\epsilon \mathcal{A}$ reduces to an $\epsilon$-oper.

Since $\epsilon$-opers are also a fundamental ingredient in the NRS conjecture, let us now give a concrete characterization of them in the simplest case of flat $\mathrm{SL}(2, \mathbb{C})$ connections. In this case $\epsilon$-opers are also known as Schrödinger operators.

$\operatorname{An} \operatorname{SL}(2, \mathbb{C}) \epsilon$-oper is defined locally by a second order ordinary differential equation

$$
\mathrm{D}_{\epsilon} \psi(z)=\epsilon^{2} \psi^{\prime \prime}(z)+q_{2}(z) \psi(z)=0,
$$

where $\psi$ is a $\left(-\frac{1}{2}\right)$-differential on $C$. That is, the operator $\mathrm{D}_{\epsilon}$ is a bundle map $\mathrm{D}_{\epsilon}$ : $K_{C}^{-1 / 2} \rightarrow K_{C}^{3 / 2}$, where $K_{C}$ is the canonical line bundle on $C$. This means that $\mathrm{D}_{\epsilon}$ is not automatically well-defined as a global object on $C$. Let us consider what happens to the differential equation under a holomorphic change of coordinates.

Under an arbitrary holomorphic coordinate change $z \mapsto z(w)$, the $\left(-\frac{1}{2}\right)$-differential $\psi$ transforms into

$$
\tilde{\psi}(w)=\psi(z(w))\left(\frac{\mathrm{d} z}{\mathrm{~d} w}\right)^{-1 / 2}
$$

\footnotetext{
${ }^{30}$ What we refer to here as a flat $\lambda$-connection is usually called a holomorphic $\lambda$-connection. However, any flat connection $\nabla$ is holomorphic in the complex structure induced by its $(0,1)$ part. In particular, the flat $\epsilon$-connection $\epsilon \mathcal{A}$ corresponds to the holomorphic $\epsilon$-connection $D_{\epsilon}=\epsilon \partial_{A}+\varphi$ in the complex structure $\bar{\partial}=\bar{\partial}_{A}+R^{2} \epsilon \bar{\varphi}$.
} 
This implies that

$$
\epsilon^{2} \tilde{\psi}^{\prime \prime}(w)=z^{\prime}(w)^{3 / 2}\left(\epsilon^{2} \psi^{\prime \prime}(z(w))-\frac{\epsilon^{2}}{2}\{\{w, z\} \psi(z(w))),\right.
$$

where the bracket $\{[\cdot, \cdot\}$ denotes the Schwarzian derivative

$$
\{\{w, z\}\}=\frac{w^{\prime \prime \prime}(z)}{w^{\prime}(z)}-\frac{3}{2}\left(\frac{w^{\prime \prime}(z)}{w^{\prime}(z)}\right)^{2}=-\frac{\{z, w\}}{z^{\prime}(w)^{2}} .
$$

Inserting these transformation laws into (4.22), we find that the differential equation changes into

$$
z^{\prime}(w)^{3 / 2}\left(\epsilon^{2} \psi^{\prime \prime}(z(w))-\frac{\epsilon^{2}}{2}\{\{w, z\}\} \psi(z(w))+z^{\prime}(w)^{-2} \tilde{q}_{2}(w) \psi(z(w))\right)=0,
$$

if $q_{2}(z)$ would simply transform into $\tilde{q}_{2}(w)$.

To ensure that the oper $\mathrm{D}_{\epsilon}$ remains invariant under arbitrary holomorphic coordinate transformations, we must require that $q_{2}(z)$ transforms as

$$
q_{2}(z) \longmapsto \tilde{q}_{2}(w)=z^{\prime}(w)^{2} q_{2}(z(w))+\frac{\epsilon^{2}}{2}\{\{z, w\}\}
$$

In other words, the coefficient function $q_{2}$ should transform as what is called a projective connection on $C$.

Another possibility would be to restrict to a coordinate atlas for which the transition functions are Möbius transformations

$$
z \longmapsto \frac{a z+b}{c z+d} \quad \text { with } \quad\left(\begin{array}{ll}
a & b \\
c & d
\end{array}\right) \in \mathrm{SL}(2, \mathbb{C}) .
$$

Under such a transformation, the Schwarzian derivative vanishes, $\{\{w, z\}=0$. Thus if we restrict to such an atlas, i.e. we fix a projective structure on $C$, the oper $\mathrm{D}_{\epsilon}$ is globally defined again, and $q_{2}(z)$ simply transforms as a quadratic differential.

The oper $\mathrm{D}_{\epsilon}$ becomes the spectral curve

$$
\Sigma: \quad w^{2}-p_{2}(z)=0
$$

in the classical limit $\epsilon \rightarrow 0$ where the projective connection $q_{2}$ becomes a quadratic differential $-p_{2}$, when we replace $\epsilon \partial_{z}$ with the momentum $w$. It is therefore known as a quantum curve. The opers $\mathrm{D}_{\epsilon}$ may also be thought of as the quantum Hamiltonians of the Hitchin integrable system (see e.g. [50]).

Example 19. Let $C=\mathbb{C}$ with an irregular singularity at $z=\infty$ (a pole of $p_{2}$ of order six, i.e. of type $L=4$ ), as in examples $1,7,10$ and 14 . The brane of $\epsilon$-opers $\mathscr{L}_{\epsilon}^{\text {oper }}$ on $C$ consists of a single point, given by the Schrödinger equation

$$
\mathrm{D}_{\epsilon} \psi(z)=\epsilon^{2} \psi^{\prime \prime}(z)-\left(z^{2}+m\right) \psi(z)=0 .
$$


The oper $\mathrm{D}_{\epsilon}$ reduces in the classical limit $\epsilon \rightarrow 0$ to the spectral curve

$$
\Sigma: \quad w^{2}=z^{2}+m
$$

Fixing the $\mathrm{SL}(2, \mathbb{C})$ gauge symmetry at infinity, as we did in example 10 , turns $m$ into a parameter on a complex one-dimensional brane of opers. In the dual Argyres-Douglas theory, this corresponds to gauging the $\mathrm{SU}(2)$ flavour symmetry of the hypermultiplet.

Example 20. Let $C=\mathbb{C}^{\times}$with irregular singularities of type $L=1$ at $z=0$ and $z=\infty$, as in examples $3,8,11,13$ and 15 . In this case there is a complex one-dimensional family of opers parametrized by the equation

$$
\mathrm{D}_{\epsilon} \psi(z)=\epsilon^{2} \psi^{\prime \prime}(z)-\left(\frac{\Lambda^{2}}{z^{3}}-\frac{2 u+\epsilon^{2} / 4}{z^{2}}+\frac{\Lambda^{2}}{z}\right) \psi(z)=0 .
$$

This equation is equivalent to the Mathieu differential equation with parameters $2 u$ and $\Lambda^{2} / 2$ (after the coordinate transformation $z=\mathrm{e}^{\mathrm{i} x}$ and the redefinition given by $\psi(z)=$ $\left.(\mathrm{i} z)^{1 / 2} \tilde{\psi}(x)\right) .{ }^{31}$ In the classical limit $\epsilon \rightarrow 0$, the oper $\mathrm{D}_{\epsilon}$ reduces to the spectral curve

$$
\Sigma: \quad w^{2}=\frac{\Lambda^{2}}{z^{3}}-\frac{2 u}{z^{2}}+\frac{\Lambda^{2}}{z} .
$$

Example 21. Let $C=\mathbb{P}_{0,1, \infty}^{1}$ with three regular punctures at $z=0,1, \infty$, which corresponds to the pair of pants building block that we discussed in section 3.2. For a fixed choice of residues $\pm m_{l} / 2$, the three-punctured sphere admits the unique quadratic differential

$$
p_{2}(z)=\frac{m_{0}^{2}}{4 z^{2}}+\frac{m_{1}^{2}}{4(z-1)^{2}}+\frac{m_{\infty}^{2}-m_{0}^{2}-m_{1}^{2}}{4 z(z-1)}
$$

with at most second order poles at all punctures. Similarly, $C$ admits a unique $\operatorname{SL}(2, \mathbb{C})$ $\epsilon$-oper given by

$$
\mathrm{D}_{\epsilon} \psi(z)=\epsilon^{2} \psi^{\prime \prime}(z)+\left(\frac{\delta_{0}}{z^{2}}+\frac{\delta_{1}}{(z-1)^{2}}+\frac{\delta_{\infty}-\delta_{0}-\delta_{1}}{z(z-1)}\right) \psi(z)=0
$$

where

$$
\delta_{l}=\frac{\epsilon^{2}-m_{l}^{2}}{4}
$$

This oper is equivalent (after a simple and standard transformation) to the classical Gauss hypergeometric differential equation.

We may turn the equation (4.22) into the first order differential equation

$$
\nabla_{\epsilon}^{\text {oper }} \Psi(z)=\epsilon \frac{\mathrm{d} \Psi(z)}{\mathrm{d} z} \mathrm{~d} z+A_{z} \mathrm{~d} z \Psi(z)=0
$$

where the 1 -jet $\Psi$ and connection coefficient $A_{z}$ are given by

$$
\Psi(z)=\left(\begin{array}{c}
-\epsilon \psi^{\prime}(z) \\
\psi(z)
\end{array}\right) \quad \text { and } \quad A_{z}=\left(\begin{array}{cc}
0 & -q_{2}(z) \\
1 & 0
\end{array}\right) .
$$

\footnotetext{
${ }^{31}$ The parametrization of the term $2 u+\epsilon^{2} / 4$ is chosen for convenience, it is not relevant in the following.
} 
This defines the oper $\mathrm{D}_{\epsilon}$ locally as a flat $\mathrm{SL}(2, \mathbb{C}) \epsilon$-connection $\nabla_{\epsilon}^{\text {oper }}$. Under a holomorphic change of coordinates $z \mapsto z(w)$, the 1 -jet $\Psi$ transforms to

$$
\tilde{\Psi}(w)=\mathrm{G}^{-1}(w) \Psi(z(w))=\left(\begin{array}{cc}
g(w)^{-1} & -\epsilon g^{\prime}(w) \\
0 & g(w)
\end{array}\right) \Psi(z(w))
$$

where $g(w)=\left(z^{\prime}(w)\right)^{-1 / 2}$, which obeys

$$
\frac{\mathrm{d} \tilde{\Psi}(w)}{\mathrm{d} w}+\tilde{A}_{w} \tilde{\Psi}(w)=0
$$

with the new connection again of the form

$$
\tilde{A}=\tilde{A}_{w} \mathrm{~d} w=\mathrm{G}^{-1} \mathrm{dG}+\mathrm{G}^{-1} A \mathrm{G}=\left(\begin{array}{cc}
0 & -\tilde{q}_{2}(w) \\
1 & 0
\end{array}\right) \mathrm{d} w .
$$

Hence the $\operatorname{SL}(2, \mathbb{C}) \epsilon$-oper defined locally by $(4.22)$ is equivalent to the flat $\operatorname{SL}(2, \mathbb{C}) \epsilon$ connection $\nabla_{\epsilon}^{\text {oper }}$ defined locally by (4.37).

In a global description, the form of the transition functions $G$ imply that $\nabla_{\epsilon}^{\text {oper }}$ is a connection on the rank two vector bundle $V_{\epsilon}$ defined as the unique extension [68]

$$
0 \longrightarrow K_{C}^{1 / 2} \longrightarrow V_{\epsilon} \longrightarrow K_{C}^{-1 / 2} \longrightarrow 0 \text {. }
$$

Rescaling the extension class gives an isomorphic bundle, so the bundles $V_{\epsilon}$ for all $\epsilon \in \mathbb{C}^{\times}$ are in fact isomorphic. In the classical limit $\epsilon \rightarrow 0$, the transition functions $G$ become diagonal, so that $\left(V_{\epsilon}, \nabla_{\epsilon}^{\text {oper }}\right)$ degenerates to a Higgs bundle $\left(V_{0}, \varphi\right)$ with rank two vector bundle $V_{0}=K_{C}^{1 / 2} \oplus K_{C}^{-1 / 2}$ and Higgs field

$$
\varphi=\left(\begin{array}{cc}
0 & -p_{2} \\
1 & 0
\end{array}\right)
$$

That is, the brane of $\epsilon$-opers $\mathscr{L}_{\epsilon}^{\text {oper }}$ is a quantization of the Hitchin section $s_{0}: \mathscr{B} \rightarrow$ $\left(\mathscr{M}_{\mathrm{H}}, J_{0}\right)$, which is defined precisely by sending the quadratic differential $p_{2}$ to the Higgs bundle $\left(V_{0}, \varphi\right)$ as above and embeds the Coulomb branch $\mathscr{B}$ in the integrable system $\left(\mathscr{M}_{\mathrm{H}}, J_{0}\right)$.

This whole story generalizes rather straightforwardly to higher rank gauge groups. For example, an $\operatorname{SL}(K, \mathbb{C}) \epsilon$-oper on $C$ corresponds to a choice of projective structure on $C$ together with $K-1$ meromorphic $k$-differentials $p=\left(p_{2}, \ldots, p_{K}\right)$, which is equivalent to a flat $\mathrm{SL}(K, \mathbb{C}) \epsilon$-connection defined locally by a linear ordinary differential equation of order $K$. This is spelled out in detail for $K=3$ in [74], where it is also shown how to obtain the brane of $\epsilon$-opers for a surface $C$ with non-maximal punctures.

Example 22. Let $K=3$ and $C=\mathbb{P}_{1, \omega, \omega^{2}}^{1}$ with three maximal singularities at the third roots of unity $z=\omega^{l}$, with $l=0,1,2$ and $\omega=\mathrm{e}^{2 \pi \mathrm{i} / 3}$, corresponding to the $E_{6}$ MinahanNemeschansky theory described in section 3.2. The complex one-dimensional brane of $\epsilon$-opers for this geometry is parametrized by the differential equation

$$
\mathrm{D}_{\epsilon} \psi(z)=\epsilon^{3} \psi^{\prime \prime \prime}(z)+\epsilon q_{2}(z) \psi^{\prime}(z)+q_{3}(z) \psi(z)=0,
$$


with

$$
q_{2}(z)=\frac{9 z}{\left(z^{3}-1\right)^{2}} \quad \text { and } \quad q_{3}(z)=\frac{u}{\left(z^{3}-1\right)^{2}}+\frac{\epsilon}{2} q_{2}^{\prime}(z) .
$$

This is called the $T_{3}$-equation in [77]. In the classical limit $\epsilon \rightarrow 0$, this brane of $\epsilon$-opers reduces to the Coulomb branch of the (massless) $E_{6}$ Minahan-Nemeschansky theory.

Curiously, in all examples that we have studied, opers corresponding to building blocks of Lagrangian $\mathcal{N}=2$ field theories are defined by well-known differential equations whose solutions are given by special functions, whereas opers corresponding to building blocks of non-Lagrangian $\mathcal{N}=2$ theories are rather unfamiliar and are not similarly characterized.

\subsection{Exact WKB analysis}

Recall that we constructed Darboux coordinates on the moduli space $\mathscr{M}_{\text {flat }}(C, \mathrm{SL}(K, \mathbb{C}))$ of flat $\operatorname{SL}(K, \mathbb{C})$ connections $\nabla$ on a (punctured) Riemann surface $C$ in section 2.4. This is easily generalized to the moduli space $\mathscr{M}_{\text {flat }}^{\epsilon}\left(C, \mathrm{SL}(K, \mathbb{C})\right.$ ) of flat $\epsilon$-connections $\nabla_{\epsilon}$ (where $\left.\epsilon \in \mathbb{C}^{\times}\right)$, as multiplying $\nabla_{\epsilon}$ by $\epsilon^{-1}$ gives an ordinary flat connection on $C$. Since any $\epsilon$-oper $\mathrm{D}_{\epsilon}$ is in particular a flat $\mathrm{SL}(K, \mathbb{C}) \epsilon$-connection $\nabla_{\epsilon}^{\text {oper }}$, it is natural to wonder how to characterize these spectral coordinates on the brane of $\epsilon$-opers.

Fix an $\epsilon$-oper $\mathrm{D}_{\epsilon}$. Consider the WKB spectral network $\mathscr{W}(u, \vartheta)$ defined by $K-1$ meromorphic $k$-differentials $u=\left(p_{2}, \ldots, p_{K}\right)$, obtained in the classical limit $\epsilon \rightarrow 0$ from $\nabla_{\epsilon}^{\text {oper }}$, and choose the phase $\vartheta=\arg \epsilon$. Then there is a distinguished $\mathscr{W}(u, \vartheta)$-abelianization which is determined by the exact WKB method, see [77] and [74, section 11].

Let us explain this point in more detail. The exact WKB method is a scheme for studying the monodromy (or bound states, or more generally Stokes data) of ordinary linear differential equations; see $[84,89,134]$ for some nice reviews. Here we focus on the case $K=2$ to simplify the notation. In this case the exact WKB method starts with the set-up at the beginning of section 4.3, that is, a holomorphic Schrödinger equation

$$
\mathrm{D}_{\epsilon} \psi(z)=\epsilon^{2} \psi^{\prime \prime}(z)+q_{2}(z ; \epsilon) \psi(z)=0
$$

on a (punctured) Riemann surface $C$, where $\psi(z)$ is a section of $K_{C}^{-1 / 2}$, and we have made the dependence of $q_{2}$ on $\epsilon$ explicit. Central to the method are exact local solutions to (4.46) of the form

$$
\psi(z)=\exp \left(\frac{1}{\epsilon} \int_{z_{0}}^{z} S\left(z^{\prime} ; \epsilon\right) \mathrm{d} z^{\prime}\right)
$$

where $z$ is a local coordinate in a contractible open subset $U \subset C$ and $z_{0} \in U$. This implies that $S(z ; \epsilon)$ obeys the Riccati equation

$$
\epsilon \partial_{z} S+S^{2}+q_{2}=0
$$

The first step in constructing $S$ is to develop a formal series solution $S^{\text {for }}$ in powers of $\epsilon$. The leading order in $\epsilon$ of the differential equation (4.48) is

$$
w^{2}+p_{2}=0
$$


which defines a branched double covering $\Sigma$ over $C$. On sheet $i=1,2$ of $\Sigma$, the higher order expansion of $S_{i}^{\text {for }}$ is then uniquely determined by (4.48) and takes the form

$$
S_{i}^{\mathrm{for}}(z ; \epsilon)=w_{i}+\sum_{n=1}^{\infty} \epsilon^{n} S_{n}(z) .
$$

This series is not convergent in general. However, using a technique called Borel resummation it can be given an analytic meaning.

Example 23. The logarithm of the gamma-function has the asymptotic expansion

$$
\log \Gamma\left(\frac{m}{\epsilon}\right)=\frac{m}{\epsilon} \log \frac{m}{\epsilon}-\frac{m}{\epsilon}-\frac{1}{2} \log \frac{m}{2 \pi \epsilon}+\sum_{g=1}^{\infty} \frac{B_{2 g}}{2 g(2 g-1)}\left(\frac{\epsilon}{m}\right)^{2 g-1}
$$

when $\epsilon \rightarrow 0$, where $B_{2 g}$ are the Bernoulli numbers. This is a divergent series, since $B_{2 g}$ grows factorially as $(2 g)$ !. The Borel transform of the series

$$
f(t)=\sum_{g=1}^{\infty} \frac{B_{2 g}}{2 g(2 g-1)} t^{2 g-1}
$$

is defined as

$$
\mathcal{B}[f](s)=\sum_{g=1}^{\infty} \frac{B_{2 g}}{(2 g-1)(2 g) !} s^{2 g-1} .
$$

The Borel sum of $f$ in the direction $\vartheta$ is then

$$
\begin{aligned}
\mathcal{S}_{\vartheta} f(t) & =\int_{0}^{\infty \mathrm{e}^{\mathrm{i} \vartheta}} \mathrm{d} s \mathrm{e}^{-s} \mathcal{B}[f](t s) \\
& =\int_{0}^{\infty \mathrm{e}^{\mathrm{i} \vartheta}} \mathrm{d} s \mathrm{e}^{-s} \sum_{g=1}^{\infty} \frac{B_{2 g}}{(2 g-1)(2 g) !}(t s)^{2 g-1} .
\end{aligned}
$$

Since $\mathcal{B}[f](s)$ has no singularity along the real $s$-axis we can choose $\vartheta=0$. The integral over $s$ then reproduces the series (4.52) since

$$
\int_{0}^{\infty} \mathrm{d} s \mathrm{e}^{-s} s^{2 g-1}=(2 g-1) !
$$

Instead, consider the integral representation of the Bernoulli numbers

$$
B_{2 g}=4 g(-1)^{g-1} \int_{0}^{\infty} \mathrm{d} z \frac{z^{2 g-1}}{\mathrm{e}^{2 \pi z}-1}
$$

for $g \geq 1$. Substituting this into (4.54) yields

$$
\begin{aligned}
\mathcal{S}_{0} f(t) & =2 \int_{0}^{\infty} \mathrm{d} s \mathrm{e}^{-s} \sum_{g=1}^{\infty} \frac{(-1)^{g-1}}{(2 g-1)(2 g-1) !} \int_{0}^{\infty} \mathrm{d} z \frac{(t s z)^{2 g-1}}{\mathrm{e}^{2 \pi z}-1} \\
& =2 \int_{0}^{\infty} \frac{\mathrm{d} s}{t} \mathrm{e}^{-s / t} \sum_{g=1}^{\infty} \frac{(-1)^{g-1}}{(2 g-1)(2 g-1) !} \int_{0}^{\infty} \mathrm{d} z \frac{(s z)^{2 g-1}}{\mathrm{e}^{2 \pi z}-1} \\
& =2 \int_{0}^{\infty} \mathrm{d} s \mathrm{e}^{-s / t} \sum_{g=1}^{\infty} \frac{(-1)^{g-1} s^{2 g-2}}{(2 g-1) !} \int_{0}^{\infty} \mathrm{d} z \frac{z^{2 g-1}}{\mathrm{e}^{2 \pi z}-1},
\end{aligned}
$$


where in the last equality we integrated by parts over $s$. Now using

$$
\frac{1}{\mathrm{e}^{s}-1}-\frac{1}{s}+\frac{1}{2}=2 \sum_{g=1}^{\infty}(-1)^{g-1} \frac{s^{2 g-1}}{(2 g-1) !} \int_{0}^{\infty} \mathrm{d} z \frac{z^{2 g-1}}{\mathrm{e}^{2 \pi z}-1}
$$

we obtain

$$
\mathcal{S}_{0} f(t)=\int_{0}^{\infty} \frac{\mathrm{d} s}{s} \mathrm{e}^{-s / t}\left(\frac{1}{\mathrm{e}^{s}-1}-\frac{1}{s}+\frac{1}{2}\right)
$$

The final formula

$$
\mathcal{S}_{0}[\log \Gamma(t)]=t \log t-t-\frac{1}{2} \log \frac{t}{2 \pi}+\mathcal{S}_{0} f\left(\frac{1}{t}\right)
$$

is known as Binet's first formula for the logarithm of the gamma-function. In this example the Borel sum is thus exact.

Going back to the Riccati equation (4.48), it is believed that the solution $S_{i}^{\text {for }}(z ; \epsilon)$, while not being convergent in general, is Borel summable in the direction $\vartheta=\arg (\epsilon)[91$, 117]. More precisely, the Borel sum $S_{i}^{\vartheta}$ gives an analytic solution of the Riccati equation away from the trajectories of the WKB spectral network $\mathscr{W}(u, \vartheta)$. Recall that these trajectories are defined by the condition

$$
\mathrm{e}^{-\mathrm{i} \vartheta} \sqrt{p_{2}(v)} \in \mathbb{R}^{\times}
$$

for any tangent vector $v$ to the trajectory; the spectral network $\mathscr{W}(u, \vartheta)$ is known as the Stokes graph in exact WKB analysis. Furthermore, $S_{i}^{\vartheta}$ has the expansion $S_{i}^{\text {for }}(z ; \epsilon)$ when $\epsilon \rightarrow 0$ while remaining in the closed half-plane with $\operatorname{Re}\left(\mathrm{e}^{-\mathrm{i} \vartheta} \epsilon\right) \geq 0$.

The Borel sum $S_{i}^{\vartheta}$ can be integrated to give an exact solution

$$
\psi_{i}^{\vartheta}(z)=\exp \left(\frac{1}{\epsilon} \int_{z_{0}}^{z} S_{i}^{\vartheta}\left(z^{\prime}\right) \mathrm{d} z^{\prime}\right)
$$

of the holomorphic Schrödinger equation (4.46). Suppose that we have two sets of solutions $\psi_{i}^{\vartheta}$ and $\tilde{\psi}_{i}^{\vartheta}$ in two neighbouring cells of $C \backslash \mathscr{W}(u, \vartheta)$ divided by a trajectory of type $i j$. Then $\psi_{i}^{\vartheta}$ and $\tilde{\psi}_{i}^{\vartheta}$ are related by the connection formulas

$$
\psi_{i}^{\vartheta} \longmapsto \tilde{\psi}_{i}^{\vartheta}=\psi_{i}^{\vartheta}+c_{i j} \psi_{j}^{\vartheta} \quad \text { and } \quad \psi_{j}^{\vartheta} \longmapsto \tilde{\psi}_{j}^{\vartheta}=\psi_{j}^{\vartheta}
$$

where the functions $c_{i j}$ are known as Stokes multipliers. Note that these connection formulas are precisely of the form of the unipotent transformations from (2.18). Hence the exact WKB solutions $\psi_{i}^{\vartheta}$ provide a basis of sections which abelianize the flat $\operatorname{SL}(2, \mathbb{C}) \epsilon$-connection $\nabla_{\epsilon}^{\text {oper }}$. The corresponding almost flat $\mathrm{GL}(1, \mathbb{C}) \epsilon$-connection $\nabla_{\epsilon}^{\mathrm{ab}}$ has the explicit form

$$
\left(\epsilon \partial_{z}-S_{i}^{\vartheta}\right) \psi_{i}^{\vartheta}=0
$$




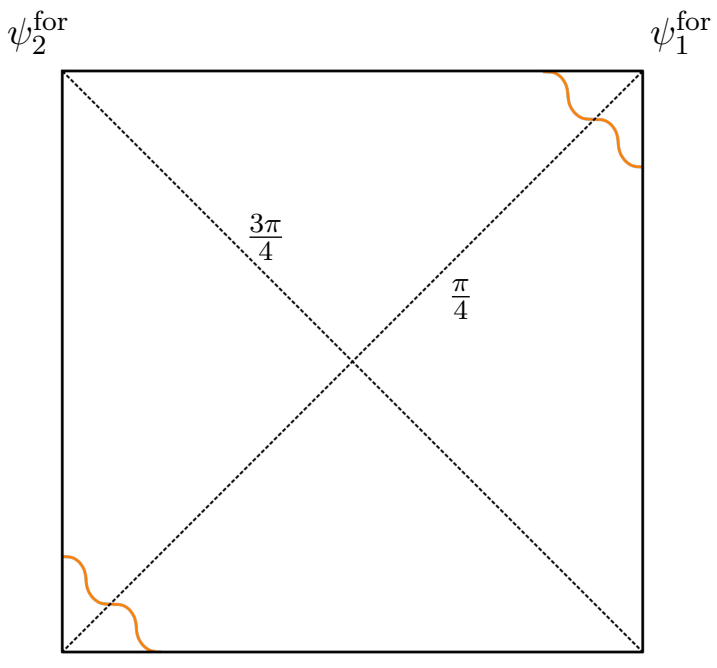

Figure 36. Stokes rays for the Schrödinger equation (4.65) with $\arg (\epsilon)=\pi / 2$.

Example 24. Perhaps the simplest example of the exact WKB method is provided by the (actual) Schrödinger equation for the complex harmonic oscillator

$$
\mathrm{D}_{\epsilon} \psi(z)=\epsilon^{2} \psi^{\prime \prime}(z)-\left(z^{2}+m\right) \psi(z)=0
$$

from example 19 [89]. Note that the differential equation (4.65) is invariant under $\epsilon \mapsto-\epsilon$ and $z \mapsto-z$. We fix $\arg (\epsilon)$. If we take $\vartheta=\arg (\epsilon)=\frac{\pi}{2}$ and $m>0$, then the corresponding spectral network $\mathscr{W}=\mathscr{W}(u, \vartheta)$ is shown in figure $3 .^{32}$ Let us choose these values for convenience. The formal series solutions to (4.65) are of the form

$$
\begin{aligned}
& \psi_{1}^{\text {for }}(z)=\mathrm{e}^{-t^{2} / 4} t^{\mu} \sum_{n=0}^{\infty}(-1)^{n} \frac{\mu(\mu-1) \cdots(\mu-2 n+1)}{n ! 2^{n} t^{2 n}}, \\
& \psi_{2}^{\text {for }}(z)=\mathrm{e}^{t^{2} / 4} t^{-\mu-1} \sum_{n=0}^{\infty} \frac{(\mu+1)(\mu+2) \cdots(\mu+2 n)}{n ! 2^{n} t^{2 n}},
\end{aligned}
$$

where

$$
t=\sqrt{\frac{2}{\epsilon}} z \quad \text { and } \quad \mu=-\frac{m}{2 \epsilon}-\frac{1}{2},
$$

and we assume $\mu \notin \mathbb{Z}$. The formal series $\psi_{1}^{\text {for }}(z)$ decreases fastest along the line $t=\sqrt{2 / \epsilon} z \in$ $\mathbb{R}$, while the formal series $\psi_{2}^{\text {for }}(z)$ decreases fastest along the line $t=\sqrt{2 / \epsilon} z \in \mathrm{i} \mathbb{R}$. These are the trajectories going to infinity at angles $\frac{\pi}{4}$ and $\frac{3 \pi}{4}$ in figure 3; see also figure 36 .

Let us therefore consider the Borel sums of $\psi_{1}^{\text {for }}(z)$ and $\psi_{2}^{\text {for }}(z)$. The Borel transform of the series

$$
f(t)=t^{\mu} \sum_{n=0}^{\infty}(-1)^{n} \frac{\mu(\mu-1) \cdots(\mu-2 n+1)}{n ! 2^{n} t^{2 n}}
$$

\footnotetext{
${ }^{32}$ Changing $\arg (\epsilon)$ rotates the trajectories at infinity; changing $\epsilon$ and $m$, while leaving $\arg (m / \epsilon)$ invariant, rotates the entire network.
} 
is given by

$$
\begin{aligned}
\mathcal{B}[f](s) & =\sum_{n=0}^{\infty}(-1)^{n} \frac{\mu(\mu-1) \cdots(\mu-2 n+1)}{n ! 2^{n} \Gamma(-\mu+2 n)} s^{2 n-\mu-1} \\
& =\sum_{n=0}^{\infty} \frac{(-1)^{n}}{n ! 2^{n} \Gamma(-\mu)} s^{2 n-\mu-1} \\
& =\frac{s^{-\mu-1}}{\Gamma(-\mu)} \mathrm{e}^{-s^{2} / 2}
\end{aligned}
$$

where the weight $\Gamma(-\mu+2 n)$ in (4.69) is determined by the prefactor $t^{\mu}$ in $\psi_{1}^{\text {for }}(z)$. The Borel sum of $f$ for $t>0$ is thus

$$
\mathcal{S}_{0} f(t)=\frac{1}{\Gamma(-\mu)} \int_{0}^{\infty} \mathrm{d} s \mathrm{e}^{-t s-\frac{s^{2}}{2}} s^{-\mu-1} .
$$

The holomorphic function

$$
\psi_{1}(z)=\mathrm{e}^{-t^{2} / 4} \mathcal{S}_{0} f(t)=D_{\mu}\left(\sqrt{\frac{2}{\epsilon}} z\right)
$$

is an integral representation of the Weber parabolic cylinder function. This is indeed an exact solution of the Schrödinger equation (4.65). The Borel sum $\psi_{1}(z)$ can be analytically continued to $\arg (z) \in\left[-\frac{\pi}{4}, \frac{3 \pi}{4}\right]$. Similarly, for $\mathrm{i} t=\sqrt{2 / \epsilon} \mathrm{i} z<0$ the Borel sum $\psi_{2}(z)$ of the formal series $\psi_{2}^{\text {for }}(z)$ coincides with the Weber parabolic cylinder function

$$
\psi_{2}(z)=\mathrm{e}^{-\frac{\pi \mathrm{i}}{2}(\mu+1)} D_{-\mu-1}\left(-\sqrt{\frac{2}{\epsilon}} \mathrm{i} z\right),
$$

and it can be analytically continued to $\arg (z) \in\left[\frac{\pi}{4}, \frac{5 \pi}{4}\right]$.

For $t=\sqrt{2 / \epsilon} z<0$ the Borel sum of $\psi_{1}^{\text {for }}(z)$ coincides with

$$
\psi_{3}(z)=\mathrm{e}^{\pi \mathrm{i} \mu} D_{\mu}\left(-\sqrt{\frac{2}{\epsilon}} z\right)
$$

and it can be analytically continued to $\arg (z) \in\left[\frac{3 \pi}{4}, \frac{7 \pi}{4}\right]$. For it $=\sqrt{2 / \epsilon} \mathrm{i} z>0$ the Borel sum of $\psi_{2}^{\text {for }}(z)$ is equal to

$$
\psi_{4}(z)=\mathrm{e}^{-\frac{\pi \mathrm{i}}{2}(3 \mu-1)} D_{-\mu-1}\left(\sqrt{\frac{2}{\epsilon}} \mathrm{i} z\right)
$$

which can be analytically continued to $\arg (z) \in\left[-\frac{3 \pi}{4}, \frac{\pi}{4}\right]$. We thus find four Stokes sectors at infinity, with bases of exact solutions as shown in figure 37 .

The exact solutions $\psi_{l}(z)$ and $\psi_{l+2}(z)$ are related across the Stokes ray labelled by $\psi_{l+1}(z)$ by the connection formula

$$
\psi_{l}=\psi_{l+2}+c_{l, l+2} \psi_{l+1}
$$

where the Stokes multipliers

$$
c_{l, l+2}=\frac{\mathrm{Wr}\left(\psi_{l}, \psi_{l+2}\right)}{\operatorname{Wr}\left(\psi_{l+1}, \psi_{l+2}\right)}
$$




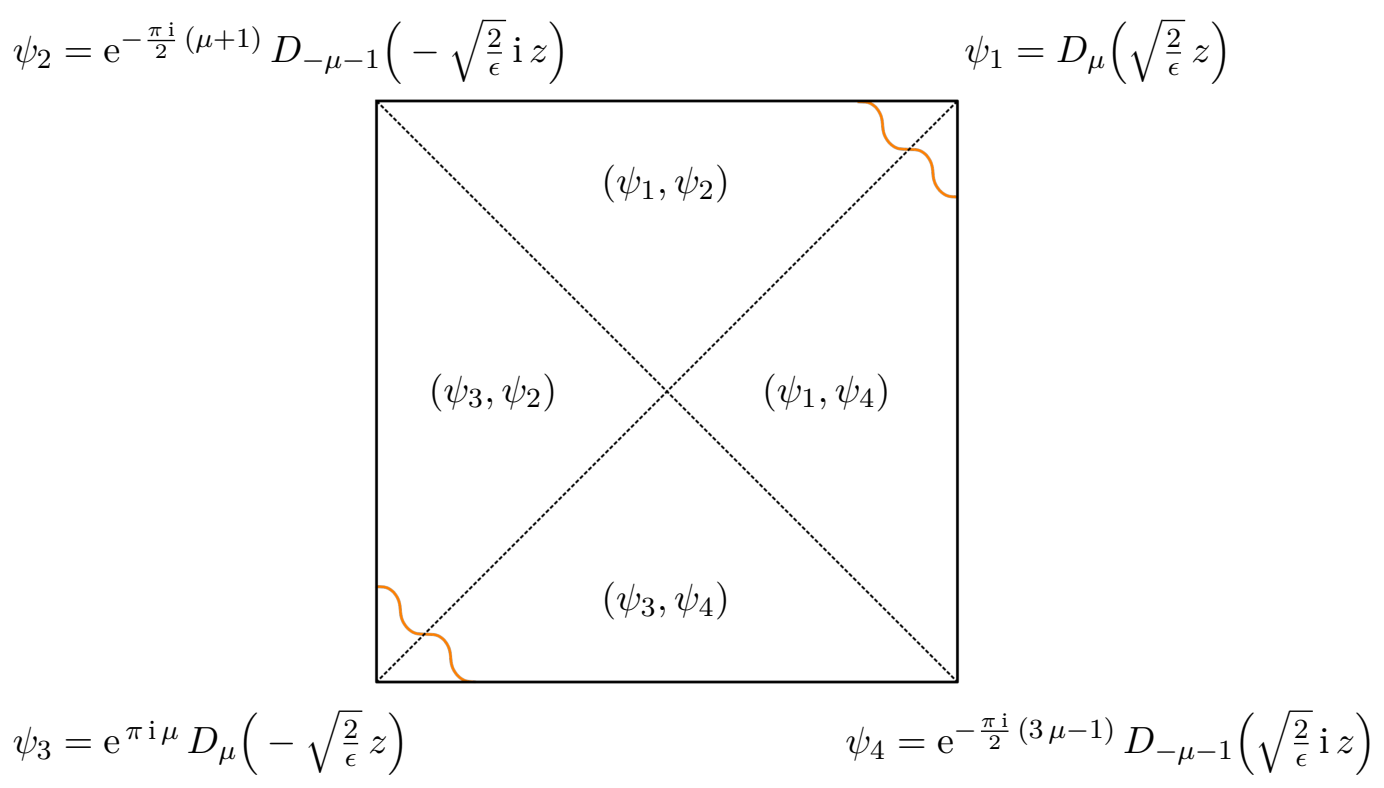

Figure 37. Local basis of solutions at infinity for the Schrödinger equation (4.65) with $\arg (\epsilon)=\pi / 2$.

may be obtained as a ratio of Wronskians of $\psi_{l}(z)$ and $\psi_{l+2}(z)$, and of $\psi_{l+1}(z)$ and $\psi_{l+2}(z)$. This yields

$$
\begin{aligned}
& \psi_{1}(z)=\psi_{3}(z)+\frac{\sqrt{2 \pi} \mathrm{e}^{\pi \mathrm{i}(\mu+1)}}{\Gamma(-\mu)} \psi_{2}(z), \\
& \psi_{2}(z)=\psi_{4}(z)-\frac{\sqrt{2 \pi} \mathrm{i} \mathrm{e}^{-2 \pi \mathrm{i} \mu}}{\Gamma(\mu+1)} \psi_{3}(z), \\
& \psi_{3}(z)=\mathrm{e}^{2 \pi \mathrm{i} \mu} \psi_{1}(z)-\frac{\sqrt{2 \pi} \mathrm{e}^{\pi \mathrm{i}(3 \mu+1)}}{\Gamma(-\mu)} \psi_{4}(z), \\
& \psi_{4}(z)=\mathrm{e}^{-2 \pi \mathrm{i} \mu} \psi_{2}(z)+\frac{\sqrt{2 \pi} \mathrm{i} \mathrm{e}^{-2 \pi \mathrm{i} \mu}}{\Gamma(\mu+1)} \psi_{1}(z),
\end{aligned}
$$

where we used $\psi_{5}(z)=\mathrm{e}^{2 \pi \mathrm{i} \mu} \psi_{1}(z)$ and $\psi_{6}(z)=\mathrm{e}^{-2 \pi \mathrm{i} \mu} \psi_{2}(z)$. These formulas indeed agree with the classical connection formulas for parabolic cylinder functions (see [120, chapter 12])

$$
\begin{aligned}
D_{\mu}(t) & =\mathrm{e}^{\mp \pi \mathrm{i} \mu} D_{\mu}(-t)+\frac{\sqrt{2 \pi}}{\Gamma(-\mu)} \mathrm{e}^{\mp \frac{\pi \mathrm{i}}{2}(\mu+1)} D_{-\mu-1}( \pm \mathrm{i} t), \\
D_{-\mu-1}( \pm \mathrm{i} t) & =-\mathrm{e}^{\mp \pi \mathrm{i} \mu} D_{-\mu-1}(\mp \mathrm{i} t)+\frac{\sqrt{2 \pi}}{\Gamma(\mu+1)} \mathrm{e}^{\mp \frac{\pi \mathrm{i}}{2} \mu} D_{\mu}(t) .
\end{aligned}
$$

Since the connection formulas (4.77) are of the form of the unipotent transformations (4.63) or (2.18), the sections

$$
s_{l}(z)=\left(\begin{array}{c}
-\epsilon \psi_{l}^{\prime}(z) \\
\psi_{l}(z)
\end{array}\right)
$$


determine a distinguished $\mathscr{W}$-abelianization of the Schrödinger oper (4.65). In particular, the exact WKB method has provided us with the framing data $s_{l}$.

Now that we have found a distinguished abelianization for the flat $\epsilon$-connection $\nabla_{\epsilon}^{\text {oper }}$, for $\arg (\epsilon)=\vartheta$, with respect to the WKB spectral network $\mathscr{W}(u, \vartheta)$, we can consider the spectral coordinates associated to this abelianization. Recall that for a one-cycle $\gamma$ on $\Sigma$, the corresponding spectral coordinate is defined by

$$
\mathcal{X}_{\gamma}^{\mathscr{W}(u, \vartheta)}\left(\nabla_{\epsilon}^{\text {oper }}\right)=\operatorname{Hol}_{\gamma} \nabla_{\epsilon}^{\mathrm{ab}} .
$$

In exact WKB analysis, the logarithm of this spectral coordinate, for $\arg (\epsilon)=\vartheta$, is known as the Voros symbol $V_{\gamma}(\epsilon)$. It follows from the discussion above that the spectral coordinate $\mathcal{X}_{\gamma}^{\mathscr{W}(u, \vartheta)}\left(\nabla_{\epsilon}^{\text {oper }}\right)$, now with $\arg (\epsilon)$ a free parameter, has good asymptotics when $\epsilon \rightarrow 0$ in the half-plane with $\operatorname{Re}\left(\mathrm{e}^{-\mathrm{i} \vartheta} \epsilon\right) \geq 0$. It is given by $\exp \left(\Pi_{\gamma}(\epsilon)\right)$, where

$$
\Pi_{\gamma}(\epsilon)=\frac{1}{\epsilon} \oint_{\gamma} S_{i}^{\text {for }}(z ; \epsilon) \mathrm{d} z
$$

is called a quantum period. ${ }^{33}$

We should point out one subtlety. The exact WKB analysis, just like abelianization, is not defined at a critical phase $\vartheta_{\text {c }}$ when the corresponding network $\mathscr{W}\left(u, \vartheta_{c}\right)$ develops one or more saddle trajectories. Again, in parallel to our approach with abelianization, one may apply the exact WKB method to the resolutions $\mathscr{W}\left(u, \vartheta_{\mathrm{c}}^{ \pm}\right)$of the critical network. In exact WKB analysis this is called lateral Borel resummation, while the average Voros symbol $V_{\gamma}^{\mathrm{c}}=\frac{1}{2}\left(V_{\gamma}^{+}+V_{\gamma}^{-}\right)$is said to be obtained using median summation.

Suppose we keep the vacuum $u$ fixed while varying the phase $\vartheta$. As long as we do not cross any BPS ray (a critical phase where the spectral network $\mathscr{W}(u, \vartheta)$ develops a saddle trajectory), the spectral coordinate $\mathcal{X}_{\gamma}^{\mathscr{W}(u, \vartheta)}\left(\nabla_{\epsilon}^{\text {oper }}\right)$ or Voros symbol $V_{\gamma}(\epsilon)$, with $\arg (\epsilon)=\vartheta$, is an analytic function of $\epsilon$.

However, the spectral coordinate $\mathcal{X}_{\gamma}^{\mathscr{W}(u, \vartheta)}\left(\nabla_{\epsilon}^{\text {oper }}\right)$ or Voros symbol $V_{\gamma}(\epsilon)$, with $\arg (\epsilon)=$ $\vartheta$, will have a jump discontinuity across a BPS ray. From the perspective of abelianization this is because the topology of the spectral network changes, while in the exact WKB method this occurs because of the need to move a contour of integration across a singularity in the Borel plane. This jump will generally be of the form of a Kontsevich-Soibelman cluster transformation associated to the BPS states supported by the BPS wall.

Hence the spectral coordinates $\mathcal{X}_{\gamma}^{\mathscr{W}(u, \vartheta)}\left(\nabla_{\epsilon}^{\text {oper }}\right)$ or Voros symbols $V_{\gamma}(\epsilon)$, with $\arg (\epsilon)=\vartheta$, are piecewise analytic functions on the parameter space. Yet, for a fixed phase $\vartheta$, these coordinates may be analytically continued to analytic functions on the whole $\epsilon$-plane. The resulting analytically continued functions agree with the spectral coordinates $\mathcal{X}_{\gamma}^{\mathscr{W}}$ obtained by the abelianization method, evaluated at the flat $\epsilon$-connection $\nabla_{\epsilon}^{\text {oper }}$, where we fix the isotopy class $\mathscr{W}$ of the spectral network $\mathscr{W}(u, \vartheta)$.

For further background, details and examples we refer to [77] and [74, section 11]. A particularly interesting feature is that the spectral coordinates $\mathcal{X}_{\gamma}^{\mathscr{W}(u, \vartheta)}\left(\nabla_{\epsilon}^{\text {oper }}\right)$ or Voros

\footnotetext{
${ }^{33}$ This is also the conformal limit of the $\zeta \rightarrow 0$ asymptotics of $\mathcal{X}_{\gamma}(\mathcal{A})$, where $\mathcal{A}$ is the flat connection from (4.19) [59].
} 


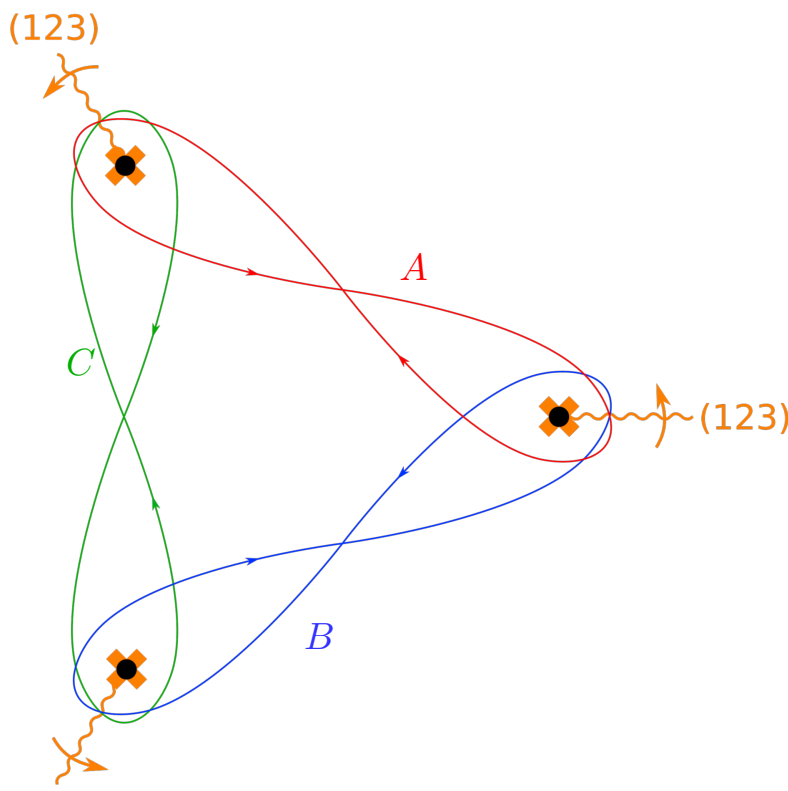

(123)

Figure 38. Three one-cycles $A, B$ and $C=-A-B$ on $\Sigma$.

periods $V_{\gamma}(\epsilon)$, for $\arg (\epsilon)=\vartheta$, may also be characterized as solutions to integral equations in the $\epsilon$-plane, called TBA equations. The general form of these equations has been formulated in [55], and derived (from reasonable analytic assumptions) in [80], while more specifics and examples are found in $[65,66,77]$ where the various methods for computing quantum periods are compared numerically. Similar equations in the context of topological string theory appear in the work of Bridgeland [25].

Example 25. Consider the one-parameter family of $\epsilon$-opers $\nabla_{\epsilon}^{\text {oper }}(u)$ from example 22 with $u \in \mathbb{R}$ and $\arg \epsilon=0=\vartheta$. For these choices the spectral network $\mathscr{W}(u, \vartheta)$ is isotopic to the circular Fenchel-Nielsen network from figure 11. We introduce one-cycles $A, B$ and $C$ with $C=-A-B$ on the spectral cover $\Sigma$ as shown in figure 38 .

The spectral coordinates $\mathcal{X}_{A}$ and $\mathcal{X}_{B}$ (for the distinguished WKB abelianization), evaluated at the family of $\epsilon$-opers $\nabla_{\epsilon}^{\text {oper }}(u)$, have an asymptotic expansion determined by the Riccati equation, as $\epsilon \rightarrow 0$ in the half-plane with $\operatorname{Re}\left(\mathrm{e}^{-\mathrm{i} \vartheta} \epsilon\right) \geq 0$. This has been verified numerically in [77, section 6.7]. The Riccati equation in this case is found by making the ansatz

$$
\psi(z ; \epsilon)=\exp \left(\sum_{n=-1}^{\infty} \epsilon^{n} \int_{0}^{z} S_{n}\left(z^{\prime}\right) \mathrm{d} z^{\prime}\right)
$$

for the solutions of the $T_{3}$-equation

with

$$
\epsilon^{3} \frac{\partial^{3} \psi(z)}{\partial z^{3}}+\epsilon q_{2}(z) \frac{\partial \psi(z)}{\partial z}+q_{3}(z) \psi(z)=0,
$$

$$
q_{2}(z)=\frac{9 z}{\left(z^{3}-1\right)^{2}} \quad \text { and } \quad q_{3}(z)=\frac{u}{\left(z^{3}-1\right)^{2}}+\frac{\epsilon}{2} q_{2}^{\prime}(z)
$$


This yields

$$
S_{-1}^{(i)}=-\frac{\omega^{i} u^{1 / 3}}{\left(z^{3}-1\right)^{2 / 3}}, \quad S_{0}^{(i)}=\frac{2 z^{2}}{z^{3}-1}, \quad S_{1}^{(i)}=\frac{\omega^{-i} u^{-1 / 3} z}{3\left(z^{3}-1\right)^{4 / 3}}, \quad \ldots
$$

for $i=0,1,2$, where we fixed the labelling of the sheets ${ }^{(i)}$ by choosing $S_{-1}^{(i)}=-\omega^{i} u^{1 / 3}$ at $z=0$, with $\omega=\mathrm{e}^{2 \pi \mathrm{i} / 3}$. The spectral coordinate $\mathcal{X}_{A}$ then has an asymptotic expansion

$$
\begin{aligned}
\mathcal{X}_{A}(\epsilon) \sim \exp \left(\Pi_{A}(\epsilon)\right) & =\exp \left(\sum_{n=-1}^{\infty} \epsilon^{n} \oint_{A} S_{n}\left(z^{\prime}\right) \mathrm{d} z^{\prime}\right) \\
& =\exp \left(\sum_{n=-1}^{\infty} \epsilon^{n}\left(\int_{1}^{\omega} S_{n}^{(2)}\left(z^{\prime}\right) \mathrm{d} z^{\prime}+\int_{\omega}^{1} S_{n}^{(3)}\left(z^{\prime}\right) \mathrm{d} z^{\prime}\right)\right) .
\end{aligned}
$$

With $t=u^{1 / 3} / \epsilon$, we find

$$
\begin{aligned}
\Pi_{A}(t)= & -\frac{\Gamma\left(\frac{1}{3}\right) \Gamma\left(\frac{1}{6}\right)}{2^{2 / 3} \sqrt{\pi}} t-\frac{3 \sqrt{\pi} \Gamma\left(\frac{5}{3}\right)}{2^{1 / 3} \Gamma\left(\frac{1}{6}\right)} t^{-1}+\frac{11 \Gamma\left(\frac{1}{3}\right) \Gamma\left(\frac{7}{6}\right)}{270 \cdot 2^{2 / 3} \sqrt{\pi}} t^{-5} \\
& -\frac{83 \sqrt{\pi} \Gamma\left(\frac{5}{3}\right)}{2268 \cdot 2^{1 / 3} \Gamma\left(\frac{7}{6}\right)} t^{-7}+\frac{44857 \Gamma\left(\frac{1}{3}\right) \Gamma\left(\frac{7}{6}\right)}{96228 \cdot 2^{2 / 3} \sqrt{\pi}} t^{-11}+\cdots
\end{aligned}
$$

Similarly, the spectral coordinates $\mathcal{X}_{B}$ and $\mathcal{X}_{C}$ have asymptotic expansions determined by the quantum periods $\Pi_{C}\left(\omega^{2} t\right)=\Pi_{B}(\omega t)=\Pi_{A}(t)$.

A challenge for the interested reader is to try to find the Borel sum $\mathcal{S}_{\vartheta} \mathcal{X}_{A}(t)$ of the quantum period $\Pi_{A}(t)$ in the direction $\vartheta$. In [77, section 6.7] it is explained how to obtain $\mathcal{X}_{A}(t)$ numerically, by both solving the abelianization problem for the $T_{3}$-equation as well as by writing down and approximating suitable integral equations. An exact answer is not presently known (and might require the definition of a new sort of "special function").

\section{The Nekrasov-Rosly-Shatashvili correspondence}

In this section we formulate a recipe for computing the effective twisted superpotential $\widetilde{\mathcal{W}}^{\text {eff }}$ of an $\mathcal{N}=2$ field theory $\mathrm{T}_{K}[C, \mathcal{D}]$ of class $\mathcal{S}$ in the $\frac{1}{2} \Omega$-background, geometrically in terms of spectral coordinates and opers. This recipe is based on a proposal of Nekrasov, Rosly and Shatashvili (NRS), who conjectured that $\widetilde{\mathcal{W}}^{\text {eff }}$ is essentially the difference between the generating functions of two holomorphic Lagrangian subspaces of the associated moduli space $\mathscr{M}_{\text {flat }}(C, \mathrm{SL}(K, \mathbb{C}))$ of flat $\mathrm{SL}(K, \mathbb{C})$ connections on $C$, in the appropriate Darboux coordinates on $\mathscr{M}_{\text {flat }}(C, \mathrm{SL}(K, \mathbb{C}))$ [114]; this conjecture is formulated in section 5.1. Evidence for this conjecture has so far been given for the conformal $\mathrm{SU}(2)$ and $\mathrm{SU}(3)$ theories coupled to four and six hypermultiplets [74, 85, 114, 137].

Here we will consider two new examples, the rank one Argyres-Douglas theory encountered in example 14 and the pure $\mathrm{SU}(2)$ theory introduced in section 3.1. The novel ingredients involved are the new Fenchel-Nielsen type coordinates found in section 2, see examples 10, 11 and 12, respectively. In section 5.2 we formulate the recipe for computing $\widetilde{\mathcal{W}}^{\text {eff }}$ geometrically. This has been tested in [74] for the conformal SU(2) and SU(3) examples. Here we test it for the rank one Argyres-Douglas theory and the weakly coupled 
pure $\mathrm{SU}(2)$ theory in sections 5.3 and 5.4, whereas in section 5.5 we use it to propose a new expansion of $\widetilde{\mathcal{W}}^{\text {eff }}$ for the $E_{6}$ Minahan-Nemeschansky theory discussed in section 3.2. In section 5.6 we give more background information and a string theory derivation of the NRS proposal, mostly following the treatment of Nekrasov and Witten in [116], while in section 5.7 we discuss the conjecture with more general boundary conditions.

\subsection{The NRS conjecture}

In section 4 we have encountered the parameter $\epsilon$ in two different contexts. Firstly, as deformation parameter of the $\frac{1}{2} \Omega$-background in section 4.1 , and secondly as a quantization parameter $\epsilon=\zeta / R$ in the Hitchin moduli space $\mathscr{M}_{\mathrm{H}}$ in sections 4.2-4.4. In [115] these two instances of $\epsilon$ are related. It is proposed that the low energy physics of the $\mathcal{N}=2$ theory $\mathrm{T}_{K}[C, \mathcal{D}]$ of class $\mathcal{S}$ in the $\frac{1}{2} \Omega$-background $\mathbb{R}_{\epsilon}^{2} \times \mathbb{R}^{2}$ is described by quantization of the Hitchin integrable system $\left(\mathscr{M}_{\mathrm{H}}(C, \mathrm{SU}(K)), J_{0}\right)$ (or more generally $\left(\mathscr{M}_{\mathrm{H}}\left(C,{ }^{\mathrm{L}} G\right), J_{0}\right)$ where ${ }^{\mathrm{L}} G$ is the Langlands dual of the gauge group $\left.G\right)$.

In particular, Nekrasov and Shatashvili argue that the generators of the 'twisted ring' of the effective two-dimensional $\mathcal{N}=(2,2)$ theory $\mathrm{T}_{\epsilon}$ may be identified with the quantum Hamiltonians, and that its supersymmetric vacua may be identified with the 'Bethe states' of the quantum integrable system. The latter claim implies that the effective twisted superpotential $\widetilde{\mathcal{W}}^{\text {eff }}$ of the $\mathcal{N}=(2,2)$ theory $\mathrm{T}_{\epsilon}$ corresponds to the Yang-Yang function $Y$ of the quantum Hitchin system. That is,

$$
\widetilde{\mathcal{W}}^{\mathrm{eff}}(a)=Y(\nu)
$$

where the vacuum expectation values $\sigma_{i}=a_{i}$ of the scalars in the twisted chiral superfields $\boldsymbol{\Sigma}$ are identified with the spectral parameters (or rapidities) $\nu_{i}$.

This proposal was made more tangible in [114]. It was conjectured that the effective twisted superpotential $\widetilde{\mathcal{W}}^{\text {eff }}$ corresponds to the difference of two generating functions of holomorphic Lagrangian submanifolds of the moduli space $\mathscr{M}_{\text {flat }}^{\epsilon}(C, \operatorname{SL}(K, \mathbb{C}))$ in appropriate Darboux coordinates, and that the supersymmetric vacua may be identified with the intersection points of these holomorphic Lagrangian submanifolds. Let us explain this in a few more words.

Given any system of holomorphic Darboux coordinates $(x, y)$ on $\mathscr{M}_{\text {flat }}^{\epsilon}(C, \operatorname{SL}(K, \mathbb{C}))$,

$$
\left\{x_{i}, y^{j}\right\}=\delta_{i}^{j},
$$

we can define a generating function $\mathcal{W}$ of any holomorphic Lagrangian submanifold of the moduli space $\mathscr{M}_{\text {flat }}^{\epsilon}(C, \mathrm{SL}(K, \mathbb{C}))$ in this coordinate chart through the equation

$$
y^{i}=\frac{\partial \mathcal{W}(x)}{\partial x_{i}},
$$

which uniquely determines it up to an $x$-independent function.

As can be anticipated from section 4, one holomorphic Lagrangian submanifold is the brane of $\epsilon$-opers $\mathscr{L}_{\epsilon}^{\text {oper }}$. As explained in section 4.3, this submanifold may be characterized as the quantization of the Coulomb branch $\mathscr{B}$ with quantization parameter $\epsilon$. The second 
holomorphic Lagrangian submanifold corresponds to a boundary condition in the $\mathcal{N}=$ $(2,2)$ theory $\mathbf{T}_{\epsilon}$ at infinity in $\mathbb{R}_{\epsilon}^{2}$. It might be surprising that such a boundary condition is needed, given that the original computation of the four-dimensional Nekrasov partition function did not require one. However, as already observed in [115], further emphasized in [116], and as we will expand on later, the two-dimensional perspective reveals that the definition of $T_{\epsilon}$ requires more data than just the choice of a four-dimensional theory $\mathrm{T}$ and a parameter $\epsilon$.

In [114] the appropriate Darboux coordinates are described in the example of the conformal SU(2) theory coupled to four hypermultiplets (which is generalized to the conformal $\mathrm{SU}(K)$ theory coupled to $2 K$ hypermultiplets in [85]). In this case the appropriate Darboux coordinates are basically the complex Fenchel-Nielsen coordinates described in example $12 .^{34}$ We will outline the string theory derivation of the NRS conjecture (based on [116]) in section 5.6. A gauge theory proof of the conjecture, in the conformal $\mathrm{SU}(2)$ and $\mathrm{SU}(3)$ examples, was given in [85], whereas a conformal field theory perspective on the conjecture was offered in [137].

\subsection{A geometric recipe}

We shall now make some additional remarks, with the goal of turning the NRS conjecture into a concrete recipe for computing the effective twisted superpotential $\widetilde{\mathcal{W}}^{\text {eff }}$ geometrically for any $\mathcal{N}=2$ theory $\mathrm{T}=\mathrm{T}_{K}[C, \mathcal{D}]$ of class $\mathcal{S}$. Recall from section 4.1 that the effective twisted superpotential $\widetilde{\mathcal{W}}^{\text {eff }}(a ; \epsilon)$ may be obtained as the Nekrasov-Shatashvili limit

$$
\widetilde{\mathcal{W}}^{\mathrm{eff}}(a ; \epsilon)=\lim _{\epsilon_{2} \rightarrow 0} \epsilon_{2} \log Z^{\mathrm{Nek}}\left(a ; \epsilon_{1}=\epsilon, \epsilon_{2}\right)
$$

of the Nekrasov partition function $Z^{\mathrm{Nek}}\left(a ; \epsilon_{1}, \epsilon_{2}\right)$. The latter partition function may be computed from first principles for any $\mathcal{N}=2$ theory $\mathrm{T}$ with a Lagrangian formulation (that is, in terms of gauge fields possibly coupled to matter fields).

However, recall that an $\mathcal{N}=2$ theory $\mathrm{T}$ may have distinct Lagrangian descriptions in different regions of its moduli space (this is the generalized S-duality discussed in section 3.2), or it may not even have a Lagrangian formulation at all (see as well section 3.2). The characterization of the corresponding two-dimensional theory $T_{\epsilon}$ (the theory $T$ in the $\frac{1}{2} \Omega$-background), as well as the explicit expression for $\widetilde{\mathcal{W}}^{\text {eff }}(a ; \epsilon)$, therefore depends on more data than just the theory $T$ and the parameter $\epsilon$. In particular, it matters in which region of the moduli space we are considering T. For example, the standard Lagrangian description of the pure $\mathrm{SU}(2)$ theory is only valid in the weak coupling region of its moduli space, and is distinct from the dual weakly coupled description near its strong coupling points. We thus obtain two distinct two-dimensional $\mathcal{N}=(2,2)$ theories $\mathrm{T}_{\epsilon}^{\mathrm{w}}$ and $\mathrm{T}_{\epsilon}^{\mathrm{s}}$ when we place either description in the $\frac{1}{2} \Omega$-background, each with its own effective twisted superpotential $\widetilde{\mathcal{W}}^{\text {eff }}$.

This extra data may be encoded in a half-BPS boundary condition at infinity in $\mathbb{R}_{\epsilon}^{2}$. This is the boundary condition that defines the second Lagrangian submanifold in the NRS conjecture. A full classification and analysis of such boundary conditions is likely to be

\footnotetext{
${ }^{34}$ These are called Darboux coordinates rather than complex Fenchel-Nielsen coordinates in [114] because the shift ambiguity in the Fenchel-Nielsen twist coordinate is fixed in the NRS Darboux coordinates.
} 
quite rich and has not been completed thus far (to our knowledge), however see [29, 43, 116] for various examples and related discussions. In particular, some simple choices have been studied in [116, section 3.4]. Especially relevant here is the type I (or standard Neumann) boundary condition, which is characterized in a four-dimensional gauge theory by Neumann boundary conditions for the components of the gauge fields parallel to the boundary, and Dirichlet boundary conditions for the component of the gauge field normal to the boundary. For an abelian gauge theory this would correspond to a Lagrangian submanifold in the Hitchin moduli space defined by the condition $\exp \left(\oint_{B} \lambda / \epsilon\right)=1$. For a non-abelian gauge theory, with a given choice of an electric-magnetic duality frame, this Lagrangian submanifold would be approximated by the condition

$$
\exp \left(\frac{1}{\epsilon} \oint_{B_{i}} \lambda\right)=1
$$

for all $i=1, \ldots, \frac{1}{2} h_{1}(\bar{\Sigma})$, near infinity on the Coulomb branch.

Here, expanding on [77], we want to speculate more generally, for any $\mathcal{N}=2$ theory $\mathrm{T}=\mathrm{T}_{K}[C, \mathcal{D}]$ of class $\mathcal{S}$, that the relevant class of half-BPS boundary conditions are labelled in the infrared by a distinguished spectral network $\mathscr{W}=\mathscr{W}\left(u, \vartheta_{c}\right)$ together with a polarization (a choice of $A$-cycles and $B$-cycles) of the Seiberg-Witten curve $\Sigma_{u}$. The spectral network $\mathscr{W}$, together with the electric-magnetic splitting, determine a choice of holomorphic exponentiated Darboux coordinates $\mathcal{X}_{\gamma}$ on the moduli space of flat $\epsilon$-connections $\mathscr{M}_{\text {flat }}^{\epsilon}\left(C, \mathrm{SL}(K, \mathbb{C})\right.$ ) (with respect to the complex structure $\left.J_{\epsilon}\right)$. In terms of these Darboux coordinates, the second Lagrangian submanifold in the NRS conjecture is given exactly by

$$
\mathcal{X}_{B_{i}}=1
$$

for all $i=1, \ldots, \frac{1}{2} h_{1}(\bar{\Sigma}) \cdot{ }^{35}$

For example, any theory $\mathrm{T}$ with a weakly coupled Lagrangian description (such as the pure $\mathrm{SU}(2)$ theory at weak coupling) comes with a canonical choice of $A$-cycles $A^{i}$ and $B$-cycles $B_{i}$ on the Seiberg-Witten curve $\Sigma_{u}$ : in a limit where an exponentiated gauge coupling $q_{i}$ tends to zero, $\exp \left(\oint_{A^{i}} \lambda\right)$ becomes exponentially small, whereas $\exp \left(\oint_{B_{i}} \lambda\right)$ becomes exponentially large. Furthermore, there is a critical phase $\vartheta_{\mathrm{c}}$ in a neighbourhood of this weak coupling point for which the $A$-cycle period satisfies $\mathrm{e}^{-\mathrm{i} \vartheta_{\mathrm{c}}} \oint_{A^{i}} \lambda<0$, and the corresponding spectral network $\mathscr{W}^{\mathrm{FN}}=\mathscr{W}\left(u, \vartheta_{\mathrm{c}}\right)$ is a Fenchel-Nielsen type network. ${ }^{36}$ The closed trajectories of this network correspond to an infinite tower of BPS particles, including the $W^{ \pm}$-bosons, accumulating at this phase.

On the other hand, suppose that $\mathrm{T}$ is an asymptotically free theory near a strong coupling point with a dual weakly coupled description. For example, say $\mathrm{T}$ is the pure $\mathrm{SU}(2)$ theory at strong coupling. ${ }^{37}$ Then the distinguished spectral network $\mathscr{W}\left(u, \vartheta_{\mathrm{c}}\right)$

\footnotetext{
${ }^{35}$ This proposal is somewhat similar in flavour to the boundary conditions described in [29, 43].

${ }^{36}$ Whenever the description of the theory $\mathrm{T}$ includes non-zero mass parameters, the isotopy class of the Fenchel-Nielsen type network depends on the values of these parameters (or better, on the wall-crossing chamber these values are part of), and is thus not quite unique. We believe these differences play no significant role in the following. See [38] for a related discussion in five dimensions where these jumps are related to flop transitions.

${ }^{37}$ This example will be treated in detail in a forthcoming paper.
} 
would be a critical Fock-Goncharov type network, with a saddle signalling the presence of a massless dyon. In this case, the massless dyon determines the $A$-cycle in the polarization of the Seiberg-Witten curve $\Sigma_{u}{ }^{38}$

Finally, suppose that $T$ is an intrinsically strongly coupled theory. For example, say $T$ is the $E_{6}$ Minahan-Nemeschansky theory. In this case we propose that the different boundary conditions are labelled by the higher rank Fenchel-Nielsen type spectral networks $\mathscr{W}\left(u, \vartheta_{c}\right)$ where the critical phase $\vartheta_{\mathrm{c}}$ satisfies $\mathrm{e}^{-\mathrm{i} \vartheta_{\mathrm{c}}} \oint_{\gamma} \lambda<0$ for a one-cycle $\gamma$ on the Seiberg-Witten curve $\Sigma_{u}$. This one-cycle also determines the polarization of $\Sigma_{u}$.

With all this in mind, we now turn the NRS conjecture into a concrete geometric recipe for computing the effective twisted superpotential $\widetilde{\mathcal{W}}^{\text {eff }}$ for any $\mathcal{N}=2$ theory $\mathrm{T}=\mathrm{T}_{K}[C, \mathcal{D}]$ of class $\mathcal{S}$ in the $\frac{1}{2} \Omega$-background, with respect to a chosen half-BPS boundary condition at infinity in the $\mathbb{R}_{\epsilon}^{2}$ plane, that is characterized by a spectral network $\mathscr{W}$ as well as a polarization of the Seiberg-Witten curve $\Sigma_{u}$. Following [74], this recipe reads:

1. Write down the brane of $\epsilon$-opers $\mathscr{L}_{\epsilon}^{\text {oper }}$ associated to the theory $\mathrm{T}$ (using the explanations of section 4.3).

2. Compute the "length" and "twist" coordinates

$$
x_{i}=\frac{\epsilon}{\pi \mathrm{i}} \log \left(-\mathcal{X}_{A^{i}}\right) \quad \text { and } \quad y^{i}=\frac{1}{2 \epsilon} \log \mathcal{X}_{B_{i}}
$$

associated to the spectral network $\mathscr{W}$ (following the instructions from section 2).

3. Evaluate the coordinates $\left(x_{i}, y^{i}\right)$ on the brane of $\epsilon$-opers $\mathscr{L}_{\epsilon}^{\text {oper }}$, with respect to the WKB-framing (as discussed in section 4.4). ${ }^{39}$

4. Finally, extract $\widetilde{\mathcal{W}}^{\text {eff }}$ through

$$
y^{i}=\frac{1}{\epsilon} \frac{\partial \widetilde{\mathcal{W}}^{\mathrm{eff}}(x ; \epsilon)}{\partial x_{i}}
$$

This recipe computes the effective twisted superpotential $\widetilde{\mathcal{W}}^{\text {eff }}$ exactly in the parameter $\epsilon$. As explained in section 4.4, the spectral coordinates $\mathcal{X}_{\gamma}$, evaluated on the brane of $\epsilon$-opers, may be interpreted as non-perturbative completions of the quantum periods $\Pi_{\gamma}(\epsilon)=\frac{1}{\epsilon}\left(\oint_{\gamma} \lambda+O(\epsilon)\right) .{ }^{40}$ The $\epsilon$-expansion for $\widetilde{\mathcal{W}}^{\text {eff }}$ may then be computed from the equations $\Pi_{B_{i}}(\epsilon)=2 \partial_{x_{i}} \widetilde{\mathcal{W}}^{\text {eff }}(x ; \epsilon)$, where for $x_{i}$ we substitute the quantum period $\epsilon \Pi_{A^{i}}(\epsilon) / \pi$ i.

For theories $\mathrm{T}$ with a weakly coupled Lagrangian formulation, this recipe computes the well-known Nekrasov-Shatashvili limit of the Nekrasov partition function with respect to the distinguished Fenchel-Nielsen type network $\mathscr{W}^{\text {FN }}$ described above. The coordinate $x_{i}$ then corresponds to a complexified Fenchel-Nielsen length parameter, which we will denote

\footnotetext{
${ }^{38}$ One might worry that there is no unique such critical Fock-Goncharov type network nor choice of $B$-cycle, but these ambiguities just illustrate the $\mathrm{SL}(2, \mathbb{Z})$ monodromy around the strong coupling point.

${ }^{39}$ Recall that there may be multiple $\mathscr{W}$-abelianizations, corresponding to distinct $\mathscr{W}$-framings.

${ }^{40}$ More precisely, $\log \mathcal{X}_{\gamma}\left(\nabla_{\epsilon}^{\text {oper }}\right)$ is an analytic continuation of the Voros symbol $V_{\gamma}(\epsilon)$ computed at $\arg (\epsilon)=$ $\vartheta$. If $\vartheta=\vartheta_{\mathrm{c}}$ is a critical phase, it is an analytic continuation of the average of the Voros symbols computed at $\arg (\epsilon)=\vartheta_{\mathrm{c}}^{ \pm}$.
} 


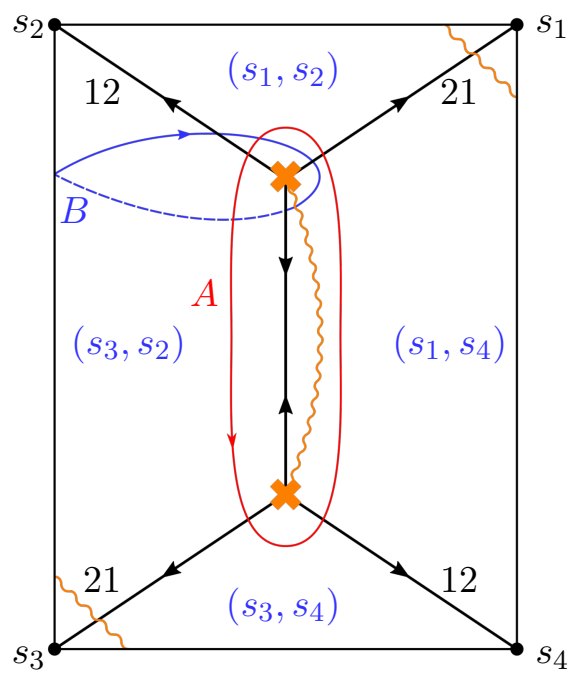

Figure 39. Critical network $\mathscr{W}$ for the $\mathrm{AD}_{2}$ theory together with a choice of $A$-cycle and $B$-cycle as well as framing data $s_{1}, s_{2}, s_{3}, s_{4}$.

in the following by $-a_{\mathrm{FN}, i}$ since it may be obtained by Borel resummation of the quantum period $\Pi_{A^{i}}(\epsilon)$ with respect to the Fenchel-Nielsen type network $\mathscr{W}^{\mathrm{FN}}$. This was already tested in [74] for the weakly coupled conformal SU(2) and $\mathrm{SU}(3)$ examples. Let us take a look at some new illustrative examples here, starting with the very simplest model of a theory of class $\mathcal{S}$.

\section{$5.3 \quad \mathrm{AD}_{2}$ theory}

Let us compute $\widetilde{\mathcal{W}}^{\text {eff }}$ for the Argyres-Douglas theory $\mathrm{AD}_{2}$ following the geometric recipe. Recall that this is the four-dimensional $\mathcal{N}=2$ field theory of a single free hypermultiplet of mass $m$, corresponding to the Seiberg-Witten curve

$$
\Sigma: \quad w^{2}=z^{2}+m,
$$

viewed as a branched covering $\Sigma \rightarrow C$ of degree two over $C=\mathbb{C}$. This geometry is illustrated in figure 31. Although the $\mathrm{AD}_{2}$ Lagrangian has merely a free hypermultiplet contribution, we can introduce gauge degrees of freedom by gauging the flavour symmetry of hypermultiplet. As we saw earlier, this turns $m$ into a variable parameterizing a complex one-dimensional Coulomb branch $\mathscr{B}$. The corresponding $A$-cycle and $B$-cycle are illustrated in figure 39 , together with the relevant critical network $\mathscr{W}$ (which we already encountered in example 1 , together with its resolutions $\mathscr{W}^{ \pm}$in example 7).

The associated complex two-dimensional moduli space $\mathscr{M}_{\text {flat }}^{\epsilon}(C, \mathrm{SL}(2, \mathbb{C}))$ of flat $\mathscr{W}$ framed $\epsilon$-connections $\nabla_{\epsilon}$ was described in example 7 , while the corresponding length and twist coordinates

$$
x=\frac{\epsilon}{\pi \mathrm{i}} \log \left(-\mathcal{X}_{A}\right) \quad \text { and } \quad y^{ \pm}=\frac{1}{2 \epsilon} \log \mathcal{X}_{B}^{ \pm}
$$


were constructed in example 10. Recall that the framing of $\nabla_{\epsilon}$ (which is the same for the network $\mathscr{W}$ and its resolutions $\mathscr{W}^{ \pm}$) consists of a local section $s_{l}$ at each of the marked points $z_{l}$ at infinity (see figure 39 ), and that the spectral coordinates

$$
\mathcal{X}_{A}=\frac{s_{1} \wedge s_{2}}{s_{3} \wedge s_{2}} \frac{s_{3} \wedge s_{4}}{s_{1} \wedge s_{4}}, \quad \mathcal{X}_{B}^{+}=\frac{s_{1} \wedge s_{4}}{s_{2} \wedge s_{4}} \quad \text { and } \quad \mathcal{X}_{B}^{-}=-\frac{s_{1} \wedge s_{3}}{s_{1} \wedge s_{2}}
$$

are determined in terms of this framing data.

The brane of $\epsilon$-opers $\mathscr{L}_{\epsilon}^{\text {oper }}$ was found to be parameterized by the Schrödinger equation

$$
\mathrm{D}_{\epsilon} \psi(z)=\epsilon^{2} \psi^{\prime \prime}(z)-\left(z^{2}+m\right) \psi(z)=0
$$

in example 19. As we saw in example 24, when $\arg (\epsilon)=\pi / 2$ and $m>0$ the spectral network $\mathscr{W}$ matches with the Stokes graph for the Schrödinger oper $\mathrm{D}_{\epsilon}$. In that case the spectral coordinates $\mathcal{X}_{A}$ and $\mathcal{X}_{B}^{ \pm}$may be computed as Voros symbols using exact WKB analysis.

Here we let $m$ and $\epsilon$ be free variables, and evaluate $\mathcal{X}_{A}$ and $\mathcal{X}_{B}^{ \pm}$on the complete family of opers $\mathrm{D}_{\epsilon}$. We frame the flat $\epsilon$-oper connection $\nabla_{\epsilon}^{\text {oper }}$ by choosing

$$
s_{l}(z)=\left(\begin{array}{c}
-\epsilon \psi_{l}^{\prime}(z) \\
\psi_{l}(z)
\end{array}\right)
$$

as in example 19. That is, the abelianization of $\nabla_{\epsilon}^{\text {oper }}$ is described in terms of the parabolic cylinder functions

$$
\begin{aligned}
& \psi_{1}(z)=D_{\mu}\left(\sqrt{\frac{2}{\epsilon}} z\right) \\
& \psi_{2}(z)=\mathrm{e}^{-\frac{\pi \mathrm{i}}{2}(\mu+1)} D_{-\mu-1}\left(-\sqrt{\frac{2}{\epsilon}} \mathrm{i} z\right), \\
& \psi_{3}(z)=\mathrm{e}^{\pi \mathrm{i} \mu} D_{\mu}\left(-\sqrt{\frac{2}{\epsilon}} z\right), \\
& \psi_{4}(z)=\mathrm{e}^{-\frac{\pi \mathrm{i}}{2}(3 \mu-1)} D_{-\mu-1}\left(\sqrt{\frac{2}{\epsilon}} \mathrm{i} z\right)
\end{aligned}
$$

with $\mu=-\frac{m}{2 \epsilon}-\frac{1}{2}$. This is the framing provided by the exact WKB method when $m>0$ and $\arg (\epsilon)=\frac{\pi}{2}$, but now analytically continued for all choices of $m$ and $\epsilon$. Since the exterior product of these sections is given by the Wronskian

$$
s_{k} \wedge s_{l}=\epsilon \operatorname{Wr}\left(\psi_{k}, \psi_{l}\right),
$$

we find

$$
\begin{aligned}
\mathcal{X}_{A}\left(\nabla_{\epsilon}^{\text {oper }}\right) & =-\exp \left(-\pi \mathrm{i} \frac{m}{\epsilon}\right), \\
\mathcal{X}_{B}^{+}\left(\nabla_{\epsilon}^{\text {oper }}\right) & =\frac{\mathrm{i}}{\sqrt{2 \pi}} \Gamma\left(\frac{1}{2}-\frac{m}{2 \epsilon}\right), \\
\mathcal{X}_{B}^{-}\left(\nabla_{\epsilon}^{\text {oper }}\right) & =\frac{\mathrm{i} \sqrt{2 \pi} \mathrm{e}^{-\pi \mathrm{i} \frac{m}{2 \epsilon}}}{\Gamma\left(\frac{1}{2}+\frac{m}{2 \epsilon}\right)} .
\end{aligned}
$$


Finally, we now have all the ingredients we need to compute $\widetilde{\mathcal{W}}^{\text {eff }}$. We define the length and twist coordinates $x$ and $y$ by averaging over the coordinates in the two resolutions (similarly to [74, section 10]) to get

$$
\begin{aligned}
& x=\frac{\epsilon}{\pi \mathrm{i}} \log \left(-\mathcal{X}_{A}\right)=-m, \\
& y=\frac{1}{2 \epsilon} \log \sqrt{\mathcal{X}_{B}^{+} \mathcal{X}_{B}^{-}}=\frac{1}{4 \epsilon} \log \frac{\Gamma\left(\frac{1}{2}+\frac{x}{2 \epsilon}\right)}{\Gamma\left(\frac{1}{2}-\frac{x}{2 \epsilon}\right)},
\end{aligned}
$$

where we dropped a linear term $\frac{1}{4 \epsilon^{2}} x$ from $y \cdot{ }^{41}$ The effective twisted superpotential $\widetilde{\mathcal{W}}^{\text {eff }}$ is then obtained by integrating the relation

$$
y=\frac{1}{\epsilon} \frac{\partial \widetilde{\mathcal{W}}^{\mathrm{eff}}(x ; \epsilon)}{\partial x} .
$$

Recall the special function from section 4.1 given by

$$
\Upsilon(x)=\int_{\frac{1}{2}}^{x} \log \frac{\Gamma\left(x^{\prime}\right)}{\Gamma\left(1-x^{\prime}\right)} \mathrm{d} x^{\prime},
$$

with the properties

$$
\frac{\partial}{\partial x} \Upsilon(a+b x)=b \log \frac{\Gamma(a+b x)}{\Gamma(1-a-b x)} \quad \text { and } \quad \Upsilon(1-x)=\Upsilon(x),
$$

for constants $a, b$. It then follows that

$$
\widetilde{\mathcal{W}}^{\mathrm{eff}}(x ; \epsilon)=\frac{\epsilon}{2} \Upsilon\left(\frac{1}{2}+\frac{x}{2 \epsilon}\right)=\frac{\epsilon}{2} \Upsilon\left(\frac{1}{2}+\frac{m}{2 \epsilon}\right),
$$

up to an integration constant that is independent of $x$. This indeed agrees with the effective twisted superpotential $\widetilde{\mathcal{W}}^{\text {eff }}(m ; \epsilon)$ from (4.11).

\subsection{Pure SU(2) theory}

As our second example, we compute the superpotential $\widetilde{\mathcal{W}}^{\text {eff }}$ for the weakly coupled pure $\mathrm{SU}(2)$ theory described in section 3.1. Recall that the Seiberg-Witten curve corresponding to the pure $\mathrm{SU}(2)$ theory is a ramified double covering $\Sigma \rightarrow C$ over the twice-punctured sphere, given by the equation

$$
w^{2}=\frac{\Lambda^{2}}{z^{3}}-\frac{2 u}{z^{2}}+\frac{\Lambda^{2}}{z}
$$

This geometry was illustrated in figure 21. In (5.22), $u$ is a parameter on the complex onedimensional Coulomb branch and $\Lambda$ is the ultraviolet scale. Since we consider the weakly coupled pure $\mathrm{SU}(2)$ theory, we assume $\left|\Lambda^{2} / u\right| \ll 1$.

In example 11 we constructed the relevant Fenchel-Nielsen coordinates on the corresponding complex two-dimensional moduli space $\mathscr{M}_{\text {flat }}^{\epsilon}(C, \mathrm{SL}(2, \mathbb{C}))$ of framed flat $\epsilon$ connections with respect to the spectral network and its resolutions, see figure $15 .{ }^{42}$ For 


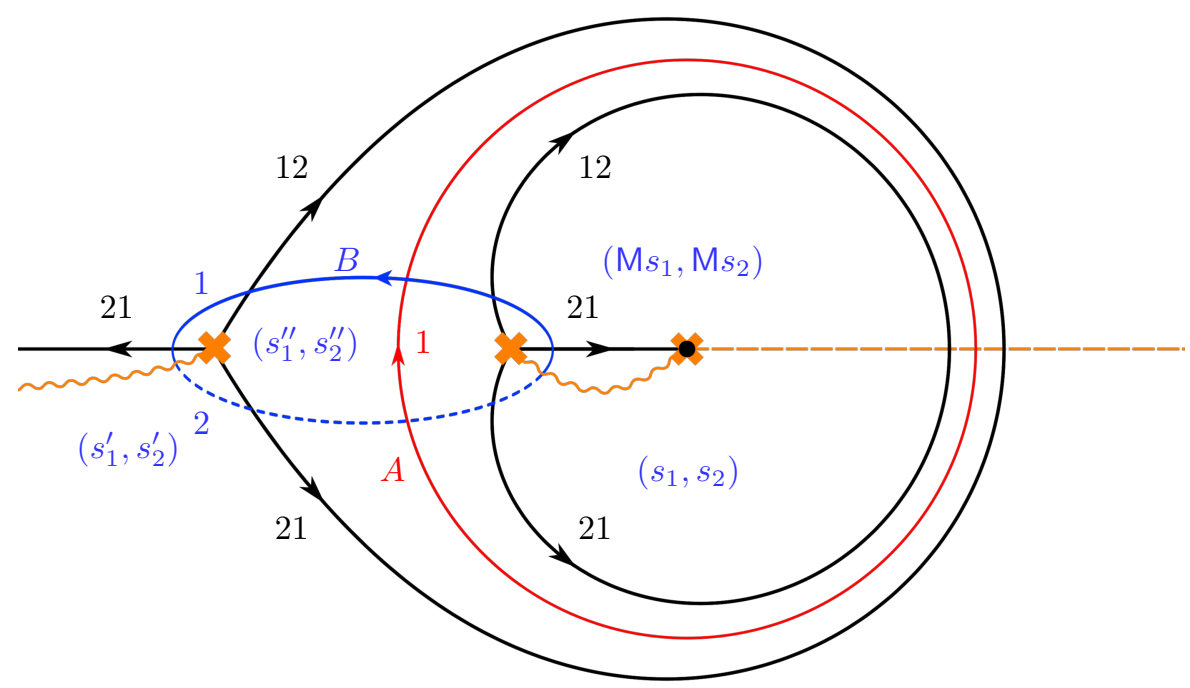

Figure 40. Fenchel-Nielsen type network for the pure SU(2) theory in the weak coupling regime together with a choice of $A$-cycle and $B$-cycle, drawn at $u=-\frac{9}{8}$ and $\vartheta=\frac{\pi}{2}$.

convenience, we have drawn this network again in figure 40. Recall that the framing data consists of a choice of local sections $s_{1} \in \ell, s_{1}^{\prime} \in \ell^{\prime}$ and $s_{2}^{\prime \prime} \in \ell^{\prime \prime}$, where $\ell$ and $\ell^{\prime}$ are $\nabla$ invariant line bundles in a neighbourhood of $z=0$ and $z=\infty$, respectively, and $\ell^{\prime \prime}$ is an eigenline of the counterclockwise monodromy $\mathrm{M}$ of $\nabla$ (see example 8).

In terms of these choices, we found in example 11 that the spectral coordinate $\mathcal{X}_{A}$ is the eigenvalue of $\mathrm{M}$ corresponding to the eigenvector $s_{2}^{\prime \prime}$, while the $B$-cycle coordinates are

$$
\begin{aligned}
& \mathcal{X}_{B}^{-}=\mathcal{X}_{A}^{2} \frac{\left(s_{1} \wedge s_{2}^{\prime \prime}\right)^{2}\left(s_{1}^{\prime} \wedge s_{1}^{\prime \prime}\right)^{2}}{\left(s_{1} \wedge s_{2}\right)\left(s_{1}^{\prime} \wedge s_{2}^{\prime}\right)\left(s_{1}^{\prime \prime} \wedge s_{2}^{\prime \prime}\right)^{2}} \\
& \mathcal{X}_{B}^{+}=\mathcal{X}_{A}^{-2} \frac{\left(s_{1} \wedge s_{2}\right)\left(s_{1}^{\prime} \wedge s_{2}^{\prime}\right)\left(s_{1}^{\prime \prime} \wedge s_{2}^{\prime \prime}\right)^{2}}{\left(s_{1} \wedge s_{1}^{\prime \prime}\right)^{2}\left(s_{1}^{\prime} \wedge s_{2}^{\prime \prime}\right)^{2}} \\
& \mathcal{X}_{B}=\sqrt{\mathcal{X}_{B}^{-} \mathcal{X}_{B}^{+}}=\frac{s_{1}^{\prime} \wedge s_{1}^{\prime \prime}}{s_{1} \wedge s_{1}^{\prime \prime}} \frac{s_{1} \wedge s_{2}^{\prime \prime}}{s_{1}^{\prime} \wedge s_{2}^{\prime \prime}}
\end{aligned}
$$

Bear in mind that the sections $s_{1}, s_{1}^{\prime}$ and $s_{2}^{\prime \prime}$ should be evaluated at the same point $z \in C$.

The brane of $\epsilon$-opers $\mathscr{L}_{\epsilon}^{\text {oper }}$ is parametrized by the Mathieu equation

$$
\mathrm{D}_{\epsilon} \psi(z)=\epsilon^{2} \frac{\partial^{2} \psi(z)}{\partial z^{2}}-\left(\frac{\Lambda^{2}}{z^{3}}-\frac{2 u+\epsilon^{2} / 4}{z^{2}}+\frac{\Lambda^{2}}{z}\right) \psi(z)=0,
$$

for $|u| \gg \Lambda^{2}$, as found in example 20. As in section 5.3, we let $\Lambda, u$ and $\epsilon$ be free variables with $\left|u / \Lambda^{2}\right| \gg 1$, and evaluate $\mathcal{X}_{A}$ and $\mathcal{X}_{B}$ on the whole family of opers $\mathrm{D}_{\epsilon}$. The local sections $s(z)$ are related to the solutions $\psi(z)$ of (5.24) as

$$
s(z)=\left(\begin{array}{c}
-\epsilon \frac{\partial \psi(z)}{\partial z} \\
\psi(z)
\end{array}\right) .
$$

\footnotetext{
${ }^{41}$ That is, we fix the shift ambiguity in the twist coordinate by defining $y$ as in (5.17).

${ }^{42}$ Although this network was drawn for specific values of $\Lambda$ and $u$, an isotopic network may be found at any point in the weak coupling region.
} 
We fix the framing data by letting $\psi_{1}(z, \Lambda)$ be the asymptotically small solution of (5.24) at $z=0$ (when approaching $z=0$ along the negative real axis), while similarly $\psi_{1}^{\prime}(z, \Lambda)$ is the asymptotically small solution at $z=\infty$ (when approaching $z=\infty$ along the negative real axis) and $\psi_{2}^{\prime \prime}(z, \Lambda)$ is the small eigenvector of the monodromy $\mathrm{M}$ of $\nabla_{\epsilon}^{\text {oper }}$, all for $-u \gg \Lambda^{2}$ and $\arg (\epsilon)=\pi / 2$. This is the framing provided by the exact WKB method for these choices of parameters, but now analytically continued to all $u, \epsilon \in \mathbb{C}$.

Unlike in section 5.3, the functions $\psi_{1}(z, \Lambda), \psi_{1}^{\prime}(z, \Lambda)$ and $\psi_{2}^{\prime \prime}(z, \Lambda)$ are not known in exact form. But it is possible to find them as series expansions in $\Lambda^{2}$, while exact in $\epsilon$. For this, we follow the approach of [74, section 9].

We start with the eigenfunctions $\psi_{i}^{\prime \prime}(z, \Lambda)$ of the monodromy M. Note that the Mathieu equation (5.24) is parametrized in terms of $\Lambda^{2}$. Since $\Lambda^{2} \ll|u|$, we expand

$$
u=\sum_{k=0}^{\infty} u_{k} \Lambda^{2 k}
$$

and

$$
\psi_{i}^{\prime \prime}(z, \Lambda)=\sum_{k=0}^{\infty} f_{k, i}^{\prime \prime}(z) \Lambda^{2 k} .
$$

At order $\Lambda^{0}$, the Mathieu equation (5.24) reads

$$
\epsilon^{2} \frac{\partial^{2} f_{0}^{\prime \prime}(z)}{\partial z^{2}}+\frac{8 u_{0}+\epsilon^{2}}{4 z^{2}} f_{0}^{\prime \prime}(z)=0
$$

with the two independent solutions

$$
f_{0, i}^{\prime \prime}(z)=\alpha_{i} z^{\frac{1}{2} \pm \frac{a_{\mathrm{FN}}}{2 \epsilon}}
$$

if we choose $u_{0}=-a_{\mathrm{FN}}^{2} / 8$. These solutions satisfy $f_{0, i}^{\prime \prime}\left(\mathrm{e}^{2 \pi \mathrm{i}} z\right)=-\mathrm{e}^{ \pm \pi \mathrm{i} a_{\mathrm{FN}} / \epsilon} f_{0, i}^{\prime \prime}(z)$; since we are looking for functions $\psi_{i}^{\prime \prime}(z, \Lambda)$ that diagonalize the monodromy around $z=0$ with eigenvalue $-\mathrm{e}^{ \pm \pi \mathrm{i} a_{\mathrm{FN}} / \epsilon}$, this is the property we require for all $f_{k, i}^{\prime \prime}(z)$. Note that if we choose $a_{\mathrm{FN}}>0$, then $\psi_{2}^{\prime \prime}(z, \Lambda)$ is indeed the small eigenvector for $-u \gg \Lambda^{2}$ and $\arg (\epsilon)=\frac{\pi}{2}$.

This guides us to the ansatz

$$
f_{k, i}^{\prime \prime}(z)=\sum_{m=-k}^{k} c_{k, \pm}^{(m)} z^{\frac{1}{2} \pm \frac{a_{\mathrm{FN}}}{2 \epsilon}+m}
$$

This ansatz indeed solves the Mathieu equation (5.24) at order $\Lambda^{2}$, which reads

$$
\epsilon^{2} \frac{\partial^{2} f_{1}^{\prime \prime}(z)}{\partial z^{2}}+\frac{8 u_{0}+\epsilon^{2}}{4 z^{2}} f_{1}^{\prime \prime}(z)-\left(\frac{1}{z^{3}}-\frac{2 u_{1}}{z^{2}}+\frac{1}{z}\right) f_{0}^{\prime \prime}(z)=0
$$

if we choose

$$
c_{1, \pm}^{(-1)}= \pm \frac{1}{\epsilon\left(a_{\mathrm{FN}} \pm \epsilon\right)}, \quad u_{1}=0 \quad \text { and } \quad c_{1, \pm}^{(1)}=\mp \frac{1}{\epsilon\left(a_{\mathrm{FN}} \mp \epsilon\right)}
$$


while $c_{1, \pm}^{(0)}$ is unconstrained. The same strategy can be applied to go to any order in $\Lambda^{2}$. For instance, at order $\Lambda^{4}$ we find

$$
\begin{aligned}
& c_{2, \pm}^{(-1)}=\frac{1}{2 \epsilon^{2}\left(a_{\mathrm{FN}} \pm 2 \epsilon\right)\left(a_{\mathrm{FN}} \pm \epsilon\right)}, \quad c_{2, \pm}^{(-1)}= \pm \frac{c_{1, \pm}^{(0)}}{\epsilon\left(a_{\mathrm{FN}} \pm \epsilon\right)}, \quad u_{2}=-\frac{1}{a_{\mathrm{FN}}^{2}-\epsilon^{2}} \\
& c_{2, \pm}^{(1)}=\mp \frac{c_{1, \pm}^{(0)}}{\epsilon\left(a_{\mathrm{FN}} \mp \epsilon\right)} \quad \text { and } \quad c_{2, \pm}^{(2)}=-\frac{1}{2 \epsilon^{2}\left(a_{\mathrm{FN}} \mp 2 \epsilon\right)\left(a_{\mathrm{FN}} \mp \epsilon\right)}
\end{aligned}
$$

while $c_{2, \pm}^{(0)}$ is again undetermined, and so on.

Computing the asymptotically small solution $\psi_{1}(z, \Lambda)$ near $z=0$ is a little trickier. This requires explicit knowledge of the expansion

$$
u=-\frac{a_{\mathrm{FN}}^{2}}{8}-\frac{\Lambda^{4}}{a_{\mathrm{FN}}^{2}-\epsilon^{2}}-\frac{5 a_{\mathrm{FN}}^{2}+7 \epsilon^{2}}{\left(a_{\mathrm{FN}}^{2}-4 \epsilon^{2}\right)\left(a_{\mathrm{FN}}^{2}-\epsilon^{2}\right)^{3}} \Lambda^{8}+O\left(\Lambda^{10}\right)
$$

which we just computed. We introduce a new coordinate $v=z / \Lambda^{2}$ which is of order 1 even if $z$ is very small. In this coordinate the Mathieu equation (5.24) reads

$$
\epsilon^{2} \frac{\partial^{2} g(v)}{\partial v^{2}}-\left(\frac{1}{v^{3}}-\frac{8 u+\epsilon^{2}}{4 v^{2}}+\frac{\Lambda^{4}}{v}\right) g(v)=0,
$$

with $g(v):=\psi\left(\Lambda^{2} v\right)$. As before, we expand $g(v)$, this time as a series in $\Lambda^{4}$ :

$$
g(v)=\sum_{l=0}^{\infty} g_{l}(v) \Lambda^{4 l}
$$

At order $\Lambda^{0}$, the rescaled Mathieu equation (5.35) reads

$$
\epsilon^{2} \frac{\partial^{2} g_{0}(v)}{\partial v^{2}}-\left(\frac{1}{v^{3}}-\frac{8 u_{0}+\epsilon^{2}}{4 v^{2}}\right) g_{0}(v)=0 .
$$

For the two independent solutions $g_{0, i}(v)$ we choose the Hankel functions

$$
g_{0, i}(v)=\sqrt{v} H^{([i+1])}\left(\frac{a_{\mathrm{FN}}}{\epsilon}, \frac{2 \mathrm{i}}{\epsilon \sqrt{v}}\right)
$$

where the index $[i+1]$ is understood modulo 2 , because they have the correct asymptotics

$$
H^{(i)}\left(\frac{a_{\mathrm{FN}}}{\epsilon}, \frac{2 \mathrm{i}}{\epsilon \sqrt{v}}\right) \simeq \sqrt{-\mathrm{i} \epsilon} v^{1 / 4} \mathrm{e}^{\mp 2 / \epsilon \sqrt{v}}
$$

when $v \rightarrow 0$ with $-\pi<\arg (2 \mathrm{i} / \epsilon \sqrt{v})<2 \pi ;^{43}$ note that $H^{(1)}$ has exponent $-\frac{2}{\epsilon \sqrt{v}}<0$ when $\arg (\epsilon)=\frac{\pi}{2}$ and $v$ has negative real part as well as negative imaginary part. It is also useful to know [1, Equation 9.1.39]

$$
H^{(2)}\left(\alpha, \mathrm{e}^{-\pi \mathrm{i}} z\right)=-\mathrm{e}^{-\pi \mathrm{i} \alpha} H^{(1)}(\alpha, z),
$$

\footnotetext{
${ }^{43}$ Note that the Hankel functions are multivalued functions, and so are really defined on the universal cover of $\mathbb{C} \backslash\{0\}$.
} 
which implies

$$
g_{0,1}\left(\mathrm{e}^{2 \pi \mathrm{i}} v\right)=\mathrm{e}^{-\pi \mathrm{i} a_{\mathrm{FN}} / \epsilon} g_{0,2}(v) .
$$

Since the solutions $\psi_{1}(z, \Lambda)$ and $\psi_{2}(z, \Lambda)$ near $z=0$ should be related as $\mathbf{M} \psi_{1}(z, \Lambda)=$ $\psi_{2}(z, \Lambda)$, this equation implies that the leading order of the solution $\psi_{i}(z, \Lambda)$ needs to be

$$
\psi_{i}(z, \Lambda)=\mathrm{e}^{ \pm \pi \mathrm{i} a_{\mathrm{FN}} / 2 \epsilon} g_{0, i}(v)+O\left(\Lambda^{4}\right) .
$$

At order $\Lambda^{4}$, the equation (5.35) reads

$$
\epsilon^{2} \frac{\partial^{2} g_{1}(v)}{\partial v^{2}}-\left(\frac{1}{v^{3}}+\frac{a_{\mathrm{FN}}^{2}+\epsilon^{2}}{v^{2}}\right) g_{1}(v)-\left(\frac{1}{v}+\frac{2}{\left(a_{\mathrm{FN}}^{2}-\epsilon^{2}\right) v^{2}}\right) g_{0}(v)=0 .
$$

Since we are looking for solutions $g(v)$ with the asymptotics (5.39) when $v \rightarrow 0$, we propose the ansatz

$$
g_{1, i}(v)=\sum_{n= \pm 1} d_{1, i}^{(n)} \sqrt{v} H^{([i+1])}\left(\frac{a_{\mathrm{FN}}}{\epsilon}+2 n, \frac{2 \mathrm{i}}{\epsilon \sqrt{v}}\right) .
$$

This indeed solves (5.43) when

$$
d_{1, i}^{(-1)}=\mp \frac{1}{a_{\mathrm{FN}} \epsilon\left(a_{\mathrm{FN}} \pm \epsilon\right)^{2}} \quad \text { and } \quad d_{1, i}^{(+1)}= \pm \frac{1}{a_{\mathrm{FN}} \epsilon\left(a_{\mathrm{FN}} \mp \epsilon\right)^{2}} .
$$

We can extend this analysis to higher orders in $\Lambda^{4}$ by making the ansatz

$$
g_{l, i}(v)=\sum_{n=-l}^{l} d_{l, i}^{(n)} \sqrt{v} H^{([i+1])}\left(\frac{a_{\mathrm{FN}}}{\epsilon}+2 n, \frac{2 \mathrm{i}}{\epsilon \sqrt{v}}\right),
$$

and solving (5.35) at order $\Lambda^{4 l}$ for $d_{l, i}^{(n)}$.

We are not done yet though, as we are looking for solutions $\psi(z)$ of the original Mathieu equation (5.24) with the correct asymptotics as $z \rightarrow 0$, in a series expansion in $\Lambda^{2}$. Thus we need to analytically continue the functions $g_{i}(v)$ to $v \rightarrow \infty$, while keeping $z=\Lambda^{2} v$ of order 1. For this, we use the fact that the Hankel functions $H^{(i)}$ have the series expansions

$$
\begin{aligned}
H^{(i)}\left(\frac{a_{\mathrm{FN}}}{\epsilon}, \frac{2 \mathrm{i}}{\epsilon \sqrt{v}}\right)= & \mp \frac{\mathrm{i}}{\pi}\left(-\epsilon^{2} v\right)^{a_{\mathrm{FN}} / 2 \epsilon} \Gamma\left(\frac{a_{\mathrm{FN}}}{\epsilon}\right)\left(1-\frac{1}{\epsilon\left(a_{\mathrm{FN}}-\epsilon\right)} v^{-1}+O\left(v^{-2}\right)\right) \\
& \pm \frac{\mathrm{i} \mathrm{e}^{\mp \frac{\pi \mathrm{i} a_{\mathrm{FN}}}{\epsilon}}\left(-\epsilon^{2} v\right)^{-a_{\mathrm{FN}} / 2 \epsilon}}{\Gamma\left(\frac{a_{\mathrm{FN}}}{\epsilon}+1\right) \sin \left(\frac{\pi a_{\mathrm{FN}}}{\epsilon}\right)}\left(1+\frac{1}{\epsilon\left(a_{\mathrm{FN}}+\epsilon\right)} v^{-1}+O\left(v^{-2}\right)\right)
\end{aligned}
$$

when $v \rightarrow \infty$. If we substitute $v=z / \Lambda^{2}$ in the corresponding series expansion in $v^{-1}$ for $g_{i}(v)$ and reorganize the resulting expression as a series in $\Lambda^{2}$, we find

$$
\psi_{i}(z, \Lambda)=\mathrm{e}^{ \pm \pi \mathrm{i} a_{\mathrm{FN}} / 2 \epsilon} g_{i}\left(z / \Lambda^{2}\right)=\sum_{k=0}^{\infty} \psi_{k, i}(z) \Lambda^{2 k},
$$

where $i \in\{1,2\}$; here $\psi_{0, i}(z)$ obtains a contribution only from the leading term in the series expansion of $g_{0, i}(v)$ when $v \rightarrow \infty$, while $\psi_{1, i}(z)$ gets a contribution from the nextto-leading term in the series expansion of $g_{0, i}(v)$ when $v \rightarrow \infty$ as well as a contribution 
from the leading term in the series expansion of $g_{1, i}(v)$ when $v \rightarrow \infty$, and so on. Note that $\psi_{1}(z, \Lambda)$ is indeed asymptotically small near $z=0$ when $\arg (\epsilon)=\frac{\pi}{2}$ and $v$ has negative real part as well as negative imaginary part.

Since the resulting function $\psi_{i}(z, \Lambda)$ should be a solution of the original Mathieu equation (5.24), we should be able to express it as a linear combination of $\psi_{1}^{\prime \prime}(z, \Lambda)$ and $\psi_{2}^{\prime \prime}(z, \Lambda)$, the eigenfunctions of the monodromy $\mathrm{M}$ computed in (5.27). Indeed we find

$$
\psi_{i}(z, \Lambda)=\frac{r_{i}}{\Lambda} \psi_{2}^{\prime \prime}(z, \Lambda)+\frac{s_{i}}{\Lambda} \psi_{1}^{\prime \prime}(z, \Lambda),
$$

where

$$
\begin{aligned}
r_{i}= & \pm \frac{\mathrm{i}}{\pi}\left(\frac{\mathrm{i} \Lambda}{\epsilon}\right)^{\frac{a_{\mathrm{FN}}}{\epsilon}} \mathrm{e}^{ \pm \frac{\pi \mathrm{i} a_{\mathrm{FN}}}{2 \epsilon}} \Gamma\left(-\frac{a_{\mathrm{FN}}}{\epsilon}\right) \\
& \times\left(1-\frac{1}{\epsilon^{2}\left(\epsilon-a_{\mathrm{FN}}\right)^{2}} \Lambda^{4}+\frac{a_{\mathrm{FN}}^{3}+a_{\mathrm{FN}}^{2} \epsilon-29 a_{\mathrm{FN}} \epsilon^{2}+43 \epsilon^{3}}{4 \epsilon^{4}\left(\epsilon+a_{\mathrm{FN}}\right)\left(2 \epsilon-a_{\mathrm{FN}}\right)^{2}\left(\epsilon-a_{\mathrm{FN}}\right)^{4}} \Lambda^{8}+O\left(\Lambda^{10}\right)\right), \\
s_{i}= & \pm \frac{\mathrm{i}}{\pi}\left(\frac{\epsilon}{\mathrm{i} \Lambda}\right)^{\frac{a_{\mathrm{FN}}}{\epsilon}} \mathrm{e}^{\mp \frac{\pi \mathrm{i} a_{\mathrm{FN}}}{2 \epsilon}} \Gamma\left(\frac{a_{\mathrm{FN}}}{\epsilon}\right) \\
& \times\left(1-\frac{1}{\epsilon^{2}\left(\epsilon+a_{\mathrm{FN}}\right)^{2}} \Lambda^{4}+\frac{-a_{\mathrm{FN}}^{3}+a_{\mathrm{FN}}^{2} \epsilon+29 a_{\mathrm{FN}} \epsilon^{2}+43 \epsilon^{3}}{4 \epsilon^{4}\left(\epsilon-a_{\mathrm{FN}}\right)\left(2 \epsilon+a_{\mathrm{FN}}\right)^{2}\left(\epsilon+a_{\mathrm{FN}}\right)^{4}} \Lambda^{8}+O\left(\Lambda^{10}\right)\right),
\end{aligned}
$$

if we set the free parameters as $c_{1, \pm}^{(0)}=c_{3, \pm}^{(0)}=0, c_{2, \pm}^{(0)}=d_{2, \pm}^{(0)}$ and $c_{4, \pm}^{(0)}=d_{4, \pm}^{(0)}$. This required computing $g_{i}(v)$ up to order $\Lambda^{16}$. As a check, note that

$$
\begin{aligned}
\mathrm{M} \psi_{1}(z, \Lambda) & =-\frac{r_{1} \mathrm{e}^{-\pi \mathrm{i} a_{\mathrm{FN}} / \epsilon}}{\Lambda} \psi_{2}^{\prime \prime}(z, \Lambda)-\frac{s_{1} \mathrm{e}^{\pi \mathrm{i} a_{\mathrm{FN}} / \epsilon}}{\Lambda} \psi_{1}^{\prime \prime}(z, \Lambda) \\
& =\frac{r_{2}}{\Lambda} \psi_{2}^{\prime \prime}(z, \Lambda)+\frac{s_{2}}{\Lambda} \psi_{1}^{\prime \prime}(z, \Lambda) \\
& =\psi_{2}(z, \Lambda) .
\end{aligned}
$$

The same strategy at the other end of $C$, where $z \rightarrow \infty$ and it is useful to introduce a local coordinate $w=\Lambda^{2} z$, shows that

$$
\psi_{i}^{\prime}(z, \Lambda)=\Lambda r_{[i+1]} \psi_{1}^{\prime \prime}(z, \Lambda)+\Lambda s_{[i+1]} \psi_{2}^{\prime \prime}(z, \Lambda) .
$$

Indeed, we check that

$$
\begin{aligned}
\mathrm{M} \psi_{1}^{\prime}(z, \Lambda) & =-\Lambda r_{2} \mathrm{e}^{\pi \mathrm{i} a_{\mathrm{FN}} / \epsilon} \psi_{1}^{\prime \prime}(z, \Lambda)-\Lambda s_{2} \mathrm{e}^{-\pi \mathrm{i} a_{\mathrm{FN}} / \epsilon} \psi_{2}^{\prime \prime}(z, \Lambda) \\
& =\Lambda r_{1} \psi_{1}^{\prime \prime}(z, \Lambda)+\Lambda s_{1} \psi_{2}^{\prime \prime}(z, \Lambda) \\
& =\psi_{2}^{\prime}(z, \Lambda)
\end{aligned}
$$

We finally have all the ingredients necessary to compute the spectral coordinates $\mathcal{X}_{A}$ and $\mathcal{X}_{B}$ at $\nabla_{\epsilon}^{\text {oper }}$, and thus to extract the effective twisted superpotential $\widetilde{\mathcal{W}}^{\text {eff }}$ in a series expansion in $\Lambda$ for the pure $\mathrm{SU}(2)$ theory at weak coupling. We find

$$
\mathcal{X}_{A}\left(\nabla_{\epsilon}^{\text {oper }}\right)=-\exp \left(-\pi \mathrm{i} \frac{a_{\mathrm{FN}}}{\epsilon}\right),
$$


while

$$
\begin{aligned}
\mathcal{X}_{B}\left(\nabla_{\epsilon}^{\text {oper }}\right) & =\frac{\operatorname{Wr}\left(\psi_{1}^{\prime}, \psi_{1}^{\prime \prime}\right)}{\operatorname{Wr}\left(\psi_{1}, \psi_{1}^{\prime \prime}\right)} \frac{\operatorname{Wr}\left(\psi_{1}, \psi_{2}^{\prime \prime}\right)}{\operatorname{Wr}\left(\psi_{1}^{\prime}, \psi_{2}^{\prime \prime}\right)}=\frac{s_{1} s_{2}}{r_{1} r_{2}}, \\
\mathcal{X}_{B}^{-}\left(\nabla_{\epsilon}^{\text {oper }}\right) & =\mathcal{X}_{A}^{2} \frac{s_{1}^{2} s_{2}^{2}}{\left(r_{1} s_{2}-r_{2} s_{1}\right)^{2}}, \\
\mathcal{X}_{B}^{+}\left(\nabla_{\epsilon}^{\text {oper }}\right) & =\mathcal{X}_{A}^{-2} \frac{\left(r_{1} s_{2}-r_{2} s_{1}\right)^{2}}{r_{1}^{2} r_{2}^{2}},
\end{aligned}
$$

where we used $s \wedge \tilde{s}=\epsilon \operatorname{Wr}(\psi, \tilde{\psi})$.

From (5.54) we find

$$
x=\frac{\epsilon}{\pi \mathrm{i}} \log \left(-\mathcal{X}_{A}\right)=-a_{\mathrm{FN}} .
$$

Substituting (5.50) into (5.55) and expanding to order $\Lambda^{8}$ we obtain

$$
\begin{aligned}
\frac{1}{2} \log \mathcal{X}_{B}= & -\frac{2 a_{\mathrm{FN}}}{\epsilon} \log \left(\frac{\Lambda}{\epsilon}\right)-\frac{1}{2} \log \frac{\Gamma\left(-\frac{a_{\mathrm{FN}}}{\epsilon}\right)}{\Gamma\left(1+\frac{a_{\mathrm{FN}}}{\epsilon}\right)} \frac{\Gamma\left(1-\frac{a_{\mathrm{FN}}}{\epsilon}\right)}{\Gamma\left(\frac{a_{\mathrm{FN}}}{\epsilon}\right)}+\frac{4 a_{\mathrm{FN}} \Lambda^{4}}{\epsilon\left(a_{\mathrm{FN}}^{2}-\epsilon^{2}\right)^{2}} \\
& +\frac{6\left(5 a_{\mathrm{FN}}^{5}-2 a_{\mathrm{FN}}^{3} \epsilon^{2}-37 a_{\mathrm{FN}} \epsilon^{4}\right)}{\epsilon\left(a_{\mathrm{FN}}^{2}-4 \epsilon^{2}\right)^{2}\left(a_{\mathrm{FN}}^{2}-\epsilon^{2}\right)^{4}} \Lambda^{8}+O\left(\Lambda^{10}\right),
\end{aligned}
$$

where we used

$$
\frac{\Gamma\left(-\frac{a_{\mathrm{FN}}}{\epsilon}\right)}{\Gamma\left(\frac{a_{\mathrm{FN}}}{\epsilon}\right)}=-\frac{\Gamma\left(1-\frac{a_{\mathrm{FN}}}{\epsilon}\right)}{\Gamma\left(1+\frac{a_{\mathrm{FN}}}{\epsilon}\right)} .
$$

The effective twisted superpotential is now obtained by integrating $\frac{1}{2} \log \mathcal{X}_{B}$ with respect to $x=-a_{\mathrm{FN}}$. This gives

$$
\begin{aligned}
\widetilde{\mathcal{W}}^{\mathrm{eff}}\left(a_{\mathrm{FN}} ; \Lambda ; \epsilon\right)= & -\int \frac{1}{2} \log \mathcal{X}_{B} \mathrm{~d} a_{\mathrm{FN}} \\
= & \frac{a_{\mathrm{FN}}^{2}}{\epsilon} \log \left(\frac{\Lambda}{\epsilon}\right)-\frac{\epsilon}{2} \Upsilon\left(-\frac{a_{\mathrm{FN}}}{\epsilon}\right)-\frac{\epsilon}{2} \Upsilon\left(\frac{a_{\mathrm{FN}}}{\epsilon}\right)+\frac{2 \Lambda^{4}}{\epsilon\left(a_{\mathrm{FN}}^{2}-\epsilon^{2}\right)} \\
& +\frac{5 a_{\mathrm{FN}}^{2}+7 \epsilon^{2}}{\epsilon\left(a_{\mathrm{FN}}^{2}-4 \epsilon^{2}\right)\left(a_{\mathrm{FN}}^{2}-\epsilon^{2}\right)^{3}} \Lambda^{8}+O\left(\Lambda^{10}\right),
\end{aligned}
$$

which indeed matches the effective twisted superpotential from (4.13) when we identify the monodromy parameter $a_{\mathrm{FN}}$ with the ( $\epsilon$-corrected) Coulomb parameter $a$. As an additional check, we compute

$$
-\epsilon \frac{\Lambda}{8} \frac{\partial \widetilde{\mathcal{W}}^{\mathrm{eff}}}{\partial \Lambda}=-\frac{a_{\mathrm{FN}}^{2}}{8}-\frac{\Lambda^{4}}{a_{\mathrm{FN}}^{2}-\epsilon^{2}}-\frac{5 a_{\mathrm{FN}}^{2}+7 \epsilon^{2}}{\left(a_{\mathrm{FN}}^{2}-4 \epsilon^{2}\right)\left(a_{\mathrm{FN}}^{2}-\epsilon^{2}\right)^{3}} \Lambda^{8}+O\left(\Lambda^{10}\right)=u,
$$

which agrees with the expansion of $u$ in $\Lambda^{2}$ given in (5.34). This is known as the quantum Matone relation $[4,14,46]$.

Finally, note that if we were to compute the effective twisted superpotential $\widetilde{\mathcal{W}}^{\text {eff }}$ with respect to the spectral coordinates $\left(\mathcal{X}_{A}, \mathcal{X}_{B}^{ \pm}\right)$, the result would only differ in the one-loop contributions. Instead of

$$
\widetilde{\mathcal{W}}_{1-\mathrm{loop}}^{\text {eff }}\left(a_{\mathrm{FN}} ; \epsilon\right)=-\frac{\epsilon}{2} \Upsilon\left(-\frac{a_{\mathrm{FN}}}{\epsilon}\right)-\frac{\epsilon}{2} \Upsilon\left(\frac{a_{\mathrm{FN}}}{\epsilon}\right)
$$


we would obtain

$$
\widetilde{\mathcal{W}}_{1-\operatorname{loop}}^{\text {eff }} \pm\left(a_{\mathrm{FN}} ; \epsilon\right)=\int\left[\mp \log \Gamma\left(\mp \frac{a_{\mathrm{FN}}}{\epsilon}\right) \mp \log \Gamma\left(1 \mp \frac{a_{\mathrm{FN}}}{\epsilon}\right)\right] \mathrm{d} a_{\mathrm{FN}}
$$

since

$$
\begin{aligned}
& \mathcal{X}_{B, 1 \text {-loop }}^{-}=\frac{1}{\pi^{2}} \Gamma\left(\frac{a_{\mathrm{FN}}}{\epsilon}\right)^{2} \Gamma\left(1+\frac{a_{\mathrm{FN}}}{\epsilon}\right)^{2}, \\
& \mathcal{X}_{B, 1 \text {-loop }}^{+}=\frac{\pi^{2}}{\Gamma\left(-\frac{a_{\mathrm{FN}}}{\epsilon}\right)^{2} \Gamma\left(1-\frac{a_{\mathrm{FN}}}{\epsilon}\right)^{2}} .
\end{aligned}
$$

Interestingly, these are the one-loop contributions to the Nekrasov partition function in the Nekrasov-Shatashvili limit, in the gauge theory or topological string scheme. ${ }^{44}$

\section{5 $\quad E_{6}$ Minahan-Nemeschansky theory}

The $E_{6}$ Minahan-Nemeschansky theory, introduced in section 3.2, is an example of an intrinsically strongly coupled $\mathcal{N}=2$ theory of class $\mathcal{S}$. Its Seiberg-Witten curve, given by (2.14), is a ramified triple covering $\Sigma \rightarrow C$ over the three-punctured sphere with three maximal punctures (see figure $25 \mathrm{~B}$ ). The three maximal punctures correspond to an $\mathrm{SU}(3) \times \mathrm{SU}(3) \times \mathrm{SU}(3)$ flavour symmetry group in the ultraviolet, which is enhanced to $E_{6}$ upon flowing to the infrared.

The $E_{6}$ theory is superconformal in the massless limit where the Seiberg-Witten curve is given by the equation

$$
w^{3}+\frac{u}{\left(z^{3}-1\right)^{2}}=0
$$

which in this case defines a triple unramified covering over the sphere with three maximal punctures at the third roots of unity $z=1, \omega, \omega^{2}$, where $\omega=\mathrm{e}^{2 \pi \mathrm{i} / 3}$. It has a onedimensional Coulomb branch $\mathscr{B}$, parametrized by $u \in \mathbb{C}$. All points on the Coulomb branch are physically equivalent. In particular, there are no walls of marginal stability where BPS bound states can form or decay. The electromagnetic charge lattice $\Gamma$ has three distinguished one-cycles, called $A, B$ and $C$ in example 25 (see figure 38). The periods $\oint_{A} \lambda=\omega \oint_{B} \lambda=\omega^{2} \oint_{C} \lambda$ are all of the same order, hence there is no canonical choice of an electric-magnetic duality frame.

In example 5 we noted that the topology of the spectral network $\mathscr{W}(u, \vartheta)$ only depends on the phase of the quantity $\mathrm{e}^{-3 \mathrm{i} \vartheta} u$, and that the network seems to be "wild" at all phases $\vartheta$, except when

$$
\mathrm{e}^{-\mathrm{i} \vartheta} \oint_{\gamma} \lambda \in \mathbb{R}
$$

for some one-cycle $\gamma$ on $\Sigma$, in which case the network is of higher rank Fenchel-Nielsen type. The simplest topology appears when $\gamma$ is equal to either of the one-cycles $A, B$ or $C$. It is illustrated in figure $11 \mathrm{~A}$ for the choice $\gamma=A$. For convenience we have drawn this network again in figure 41. (The networks corresponding to $\gamma=B, C$ are found simply by 


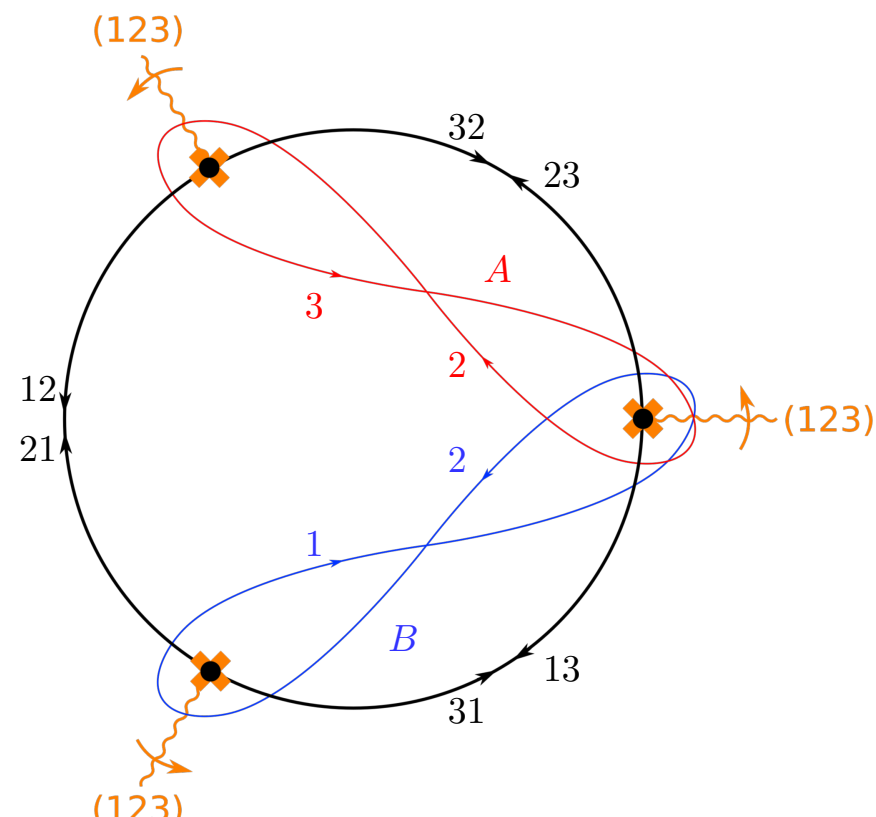

Figure 41. Higher rank Fenchel-Nielsen network for the $E_{6}$ Minahan-Nemeschansky theory together with a choice of $A$-cycle and $B$-cycle.

rotating this network through $120^{\circ}$.) The Fenchel-Nielsen coordinates $\mathcal{X}_{A}$ and $\mathcal{X}_{B}$ for the circular network $\mathscr{W}_{[1,0]}$ illustrated in figure $11 \mathrm{~A}$ have been obtained and studied in [77], however in a form in which it is not easy to extract explicit expressions.

The effective twisted superpotential $\widetilde{\mathcal{W}}^{\text {eff }}$ for the $E_{6}$ theory is obtained by evaluating the coordinates $\mathcal{X}_{A}$ and $\mathcal{X}_{B}$ on the family of $T_{3}$-opers $\nabla_{\epsilon}^{\text {oper }}(u)$ written down in example 22. Even though it is difficult to obtain exact expressions in $\epsilon$ for this example, we have learned in example 25 that the spectral coordinates $\mathcal{X}_{A}$ and $\mathcal{X}_{B}$ at $\nabla_{\epsilon}^{\text {oper }}(u)$ have an asymptotic expansion, as $\epsilon \rightarrow 0$ in the half-plane with $\operatorname{Re}\left(\mathrm{e}^{-\mathrm{i} \vartheta} \epsilon\right) \geq 0$, determined by the Riccati equation. With the expansion (4.87), and by defining $\widetilde{\mathcal{W}}^{\text {eff }}$ through

$$
x=\frac{\epsilon}{\pi \mathrm{i}} \log \left(-\mathcal{X}_{A}\right) \quad \text { and } \quad \frac{\partial \widetilde{\mathcal{W}}^{\text {eff }}}{\partial x}=\frac{1}{2} \log \mathcal{X}_{B}
$$

we find

$$
\begin{aligned}
\widetilde{\mathcal{W}}^{\text {eff }}(x ; \epsilon)= & -\frac{\pi^{2}}{4 \epsilon} \mathrm{e}^{4 \pi \mathrm{i} / 3} x^{2}+6 \pi \mathrm{i} \epsilon \log \frac{x}{\epsilon}+\frac{12 \sqrt{3} \mathrm{i} \epsilon^{3}}{x^{2}}-\frac{144 \mathrm{i} \epsilon^{5}}{\pi x^{4}} \\
& +\frac{2 \sqrt{3}\left(16800 \pi^{13 / 2}+23 \cdot 2^{2 / 3} \Gamma\left(\frac{1}{3}\right)^{8} \Gamma\left(\frac{7}{6}\right)^{5}\right) \epsilon^{7}}{35 \pi^{17 / 2} x^{6}}+\cdots
\end{aligned}
$$

in an expansion in $\epsilon$. Solving the challenge posed in example 25, that is, finding the Borel sum $\mathcal{S}_{\vartheta} \mathcal{X}_{A}(t)$, would enable one to write down the exact effective twisted superpotential $\widetilde{\mathcal{W}}^{\text {eff }}$ in $\epsilon$ for the $E_{6}$ theory.

\footnotetext{
${ }^{44}$ Recall that the one-loop contribution exp $\widetilde{\mathcal{W}}_{1-l o o p}^{\text {eff }}$ is only determined up to a phase, see Footnote 25.
} 


\subsection{Derivation from quantum field theory}

Now that we have explained how the geometric recipe works, let us take a closer look at the physics behind it. We start by sketching a derivation of the NRS correspondence from the point of view of quantum field theory, mostly following the treatments of $[114,116]$.

Consider a theory $\mathrm{T}=\mathrm{T}_{\mathfrak{g}}[\mathrm{C}, \mathcal{D}]$ of class $\mathcal{S}$ in the $\frac{1}{2} \Omega$-background $\mathbb{R}_{\epsilon}^{2} \times S^{1} \times \mathbb{R}$. On the one hand, as we reviewed in section 4.1, Nekrasov and Shatashvili argued in [115] that the resulting theory $\mathbf{T}_{\epsilon}$ in the infrared limit may be described as a two-dimensional sigmamodel (with worldsheet $S^{1} \times \mathbb{R}$ ) into the complexified maximal torus of the gauge group $G$ with the effective twisted superpotential $\widetilde{\mathcal{W}}^{\text {eff }}(a ; \epsilon)$.

On the other hand, as we will describe here, an alternative three-dimensional sigmamodel argument shows that this superpotential is also equal to the difference of the generating functions of two holomorphic Lagrangian submanifolds of the moduli space $\mathscr{M}_{\text {flat }}^{\epsilon}\left(C,{ }^{\mathrm{L}} G_{\mathbb{C}}\right)$ of flat ${ }^{\mathrm{L}} G_{\mathbb{C}} \epsilon$-connections on the Riemann surface $C$, in suitable Darboux coordinates. Pivotal in this argument is the observation in [116] that the precise metric on $\mathbb{R}_{\epsilon}^{2}$ is not essential, as long as it is $\mathrm{U}(1)$-invariant, so that we may equivalently replace it with a "cigar" metric of the form

$$
\mathrm{d} s^{2}=\mathrm{d} r^{2}+f(r) \mathrm{d} \theta^{2},
$$

with $0 \leq r<\infty$ and $0 \leq \theta<2 \pi$, where $f(r) \simeq r^{2}$ for $r \rightarrow 0$ and $f(r) \rightarrow \rho^{2}$ as $r \rightarrow \infty$. We write $D$ for $\mathbb{R}^{2}$ endowed with such a cigar metric, and $D_{R}$ for $D$ restricted to $r \leq R$, with $R$ larger than the radius $r_{0}$ where the circle parametrized by $\theta$ reaches its asymptotic radius $\rho$.

Since we take $R$ to be very large, $D_{R}$ looks globally like $I \times \widetilde{S}^{1}$, with the interval $I$ parametrized by $0 \leq r \leq R$ and the circle $\widetilde{S}^{1}$ by $\theta$. We then turn on a $\frac{1}{2} \Omega$-deformation with parameter $\epsilon$ corresponding to the $\mathrm{U}(1)$ isometry of $D_{R}$ generated by $\frac{\partial}{\partial \theta}$. We denote the resulting theory, that is, the theory $\mathrm{T}$ in the $\frac{1}{2} \Omega$-background $D_{R, \epsilon} \times \mathbb{R}^{2}$, by the same symbol $\mathrm{T}_{\epsilon}$.

We now compactify the theory $\mathrm{T}_{\epsilon}$ over the fiber $\widetilde{S}^{1}$ of the cigar $D_{R, \epsilon}$. As demonstrated by [116], the $\frac{1}{2} \Omega$-deformation can be undone away from the tip of the cigar, where $r=$ 0 , in exchange for a field redefinition and setting the asymptotic radius to $\rho=|\epsilon|^{-1}$. Then, away from the tip of the cigar, compactification of the $\frac{1}{2} \Omega$-deformed theory $\mathbf{T}_{\epsilon}$ gives the same result as compactification of the undeformed theory, but now with field and coupling redefinitions.

Following the arguments of section 4.2, we may thus describe the theory $\mathrm{T}_{\epsilon}$ at low energies $E \ll|\epsilon|$, at least away from the tip of the cigar, as a three-dimensional $\mathcal{N}=4$ sigmamodel, whose target space is the hyperkähler Hitchin moduli space $\mathscr{M}_{\mathrm{H}}(C, G)$. In [116] it is then argued that the complete result, after compactifying the theory $\mathrm{T}_{\epsilon}$ over the fiber $\widetilde{S}^{1}$ of the cigar and then T-dualizing along either circle $\widetilde{S}^{1}$ or $S^{1},{ }^{45}$ is a conventional three-dimensional $\mathcal{N}=4$ sigma-model with worldvolume $I \times S^{1} \times \mathbb{R}$ into either $\mathscr{M}_{\mathrm{H}}(C, G)$

\footnotetext{
${ }^{45}$ Recall from section 4.2 that to arrive at the conventional description of this sigma-model in terms of hypermultiplets (rather than linear multiplets), it is necessary to dualize the abelian gauge fields in order to obtain enough periodic scalars. Alternatively, one may perform T-duality on either fiber $S^{1}$ or $\widetilde{S}^{1}$.
} 
or $\mathscr{M}_{\mathrm{H}}\left(C,{ }^{\mathrm{L}} G\right)$ (the Hitchin moduli space for the Langlands dual gauge group ${ }^{\mathrm{L}} G$ ). This sigma-model comes with two branes: a brane $\mathscr{L}^{\prime}$ at $r=0$ which arises purely from the geometry at the tip of the cigar, and a brane $\mathscr{L}$ at $r=R$ which descends from a choice of half-BPS boundary condition in the four-dimensional theory.

If we choose to T-dualize along the circle $\widetilde{S}^{1}$, the brane $\mathscr{L}^{\prime}$ at $r=0$ is the space-filling (or canonical) coisotropic brane from [88]. On the other hand, T-dualizing along the circle $S^{1}$ results in its three-dimensional mirror brane, the brane of $\epsilon$-opers $\mathscr{L}_{\epsilon}^{\text {oper }}$ (also described in [88] and introduced in section 4.3 of these notes). The latter is obviously the interesting choice for us.

As proposed in section 5.2, we assume that the brane $\mathscr{L}=\mathscr{L}_{\mathscr{W}, \Pi}$ at $r=R$ is labelled by the distinguished spectral network $\mathscr{W}=\mathscr{W}\left(u, \vartheta_{c}\right)$ together with a polarization $\Pi=$ $\left\{A^{i}, B_{i}\right\}$ of the Seiberg-Witten curve $\Sigma_{u}$. That is, it corresponds to the holomorphic Lagrangian submanifold of the Hitchin moduli space defined by the equations

$$
\mathcal{X}_{B_{i}}^{\mathscr{W}}=1
$$

in the system of holomorphic exponentiated Darboux coordinates $\left\{\mathcal{X}_{A^{i}}^{\mathscr{W}}, \mathcal{X}_{B_{i}}^{\mathscr{W}}\right\}$ obtained by abelianization with respect to $\mathscr{W}$. The brane of $\epsilon$-opers $\mathscr{L}_{\epsilon}^{\text {oper }}$ is instead defined by its generating function $\mathcal{W}^{\text {oper }}(\epsilon)$ in these coordinates. ${ }^{46}$

This implies that, when the interval $I$ is contracted to a point, the three-dimensional $\mathcal{N}=4$ sigma-model with target space $\mathscr{M}_{\mathrm{H}}\left(C,{ }^{\mathrm{L}} G\right)$ may be equivalently described as a twodimensional $\mathcal{N}=(2,2)$ sigma-model with target space $\left(\mathbb{C}^{\times}\right)^{r}$. This sigma-model is then characterized by a twisted superpotential ${ }^{47}$

$$
\widetilde{\mathcal{W}}^{\sigma}=\epsilon \mathcal{W}^{\text {oper }}(\epsilon)
$$

Hence, comparing the two arguments, we conclude that the effective twisted superpotential $\widetilde{\mathcal{W}}^{\text {eff }}$ characterizing the original $\mathcal{N}=2$ field theory $\mathrm{T}_{\epsilon}$ may be identified with the generating function $\mathcal{W}^{\text {oper }}(\epsilon)$ for the brane of $\epsilon$-opers $\mathscr{L}_{\epsilon}^{\text {oper }}$. The vacua of the theory $\mathrm{T}_{\epsilon}$ correspond to the points of intersection $\mathscr{L}_{\epsilon}^{\text {oper }} \cap \mathscr{L}$.

Another check of the NRS correspondence would be to compare vacuum expectation values of half-BPS line defects in the theory $\mathrm{T}_{\epsilon}$. Suppose we consider such line defects wrapping the fiber $\widetilde{S}^{1}$ of the cigar geometry $D_{R, \epsilon}$ while being situated at a point of $\mathbb{R}^{2}$. In the ultraviolet these line defects are labelled by a loop or network on $C$ as well as a phase $\zeta=\mathrm{e}^{\mathrm{i} \vartheta}$ (see e.g. [47, 57]). Their vacuum expectation values in an infrared vacuum $u$ have

\footnotetext{
${ }^{46}$ In order to preserve four supersymmetries in the three-dimensional sigma-model, it is necessary to choose $\arg (\epsilon)=\vartheta$. However, we can analytically continue all of our results, and in particular the generating function $\mathcal{W}^{\text {oper }}(\epsilon)$, to any complex $\epsilon$. Indeed, recall from section 4.4 that the spectral coordinates $\mathcal{X}_{\gamma}^{\mathscr{W}}$, evaluated on the family of flat $\epsilon$-connections $\nabla_{\epsilon}^{\text {oper }}$, are equal to the Voros periods $\exp V_{\gamma}(\epsilon)$ when $\arg (\epsilon)=\vartheta$, but then analytically continued to all $\epsilon \in \mathbb{C}^{\times}$.

${ }^{47} \mathrm{~A}$ similar statement holds in topologically twisted sigma-models, for example in the reduction of the twodimensional A-model with worldsheet $I \times \mathbb{R}$ to quantum mechanics on $\mathbb{R}$, where this twisted superpotential is the Morse function generating the Fukaya category, or in the three-dimensional Rozansky-Witten model, which is explained in [87]. These arguments are generalized to three-dimensional $\mathcal{N}=4$ gauge theories in $[28]$.
} 
natural expansions

$$
\left\langle L_{\zeta}\right\rangle_{u}=\sum_{\gamma \in \Gamma_{u}} \underline{\bar{\Omega}}\left(\gamma, L_{\zeta} ; u\right) \mathcal{X}_{\gamma}^{\mathscr{W}(u, \vartheta)}
$$

in terms of the spectral coordinates $\mathcal{X}_{\gamma}^{\mathscr{W}(u, \vartheta)}$ obtained by abelianization with respect to the spectral network $\mathscr{W}(u, \vartheta)$. Here $\underline{\bar{\Omega}}\left(\gamma, L_{\zeta} ; u\right)$ are indices which count what are called the framed BPS states of charge $\gamma$ supported by the line defect $L_{\zeta}$.

In order to preserve four supersymmetries in the theory $\mathrm{T}_{\epsilon}$, the phase $\zeta$ of the line defect $L_{\zeta}$ has to be chosen consistently with the boundary conditions $\mathscr{L}_{\epsilon}^{\text {oper }}$ and $\mathscr{L}_{\mathscr{W}, \Pi}$ (see Footnote 46). The vacuum expectation value of the resulting half-BPS line defect $L_{\epsilon}$ has an expansion

$$
\left\langle L_{\epsilon}\right\rangle_{u}=\sum_{\gamma \in \Gamma_{u}} \underline{\bar{\Omega}}\left(\gamma, L_{\epsilon} ; u\right) \mathcal{X}_{\gamma}^{\mathscr{W}},
$$

in terms of the Darboux coordinates $\mathcal{X}_{\gamma}^{\mathscr{W}}$ corresponding to the distinguished spectral network $\mathscr{W}$. That is, for a weakly coupled Lagrangian theory $\mathrm{T}_{\epsilon}$, vacuum expectation values of half-BPS line defects have a natural expansion in terms of complex Fenchel-Nielsen coordinates.

The NRS correspondence would thus be verified if we could perform an analogous computation in the four-dimensional field theory, for example by computing the vacuum expectation value $\left\langle L_{\epsilon}\right\rangle_{u}$ using supersymmetric localization in the $\frac{1}{2} \Omega$-background. Although similar localization computations for weakly coupled Lagrangian theories indeed yield the correct expansion in terms of complex Fenchel-Nielsen coordinates, as far as we are aware the relevant calculation in the $\frac{1}{2} \Omega$-background has yet to be done. ${ }^{48}$

\subsection{A more general effective twisted superpotential}

In section 5.2 we argued that the Lagrangian submanifold $\mathscr{L}_{\mathscr{W}, \Pi}$ corresponds to the type I (or standard Neumann) boundary condition of [116], at least when $\mathbf{T}_{\epsilon}$ is a weakly coupled gauge theory. A more general boundary condition may be obtained by coupling the fourdimensional theory $\mathbf{T}_{\epsilon}$ to three-dimensional degrees of freedom on its boundary. In [42, 43] it is argued that the three-dimensional $\mathcal{N}=2$ theories $\mathrm{T}_{K}[M]$ of class $\mathcal{R}$ form a natural class of such boundary conditions. These superconformal field theories have ultraviolet Lagrangian descriptions as abelian Chern-Simons-matter theories, possibly deformed by superpotential terms that may contain monopole operators [40-42].

Similarly to the $\mathcal{N}=2$ theories $\mathrm{T}_{K}[C]$ of class $\mathcal{S}$ (see section 3.2), these three-dimensional theories may be obtained from a (twisted) compactification of the six-dimensional

\footnotetext{
${ }^{48}$ The supersymmetric localization calculations available $[81,119]$ indeed express the vacuum expectation values $\left\langle L_{\epsilon}\right\rangle_{u}$ in terms of complex Fenchel-Nielsen coordinates for weakly coupled Lagrangian field theories $\mathrm{T}_{\epsilon}$ and might appear very suggestive. See also [24] for a similar investigation. However, beware that these supersymmetric localization computations are made in the $\Omega$-background denoted by $S^{1} \times{ }_{b} \mathbb{R}^{3}$. This is an $\Omega$-background with a pair of parameters $\left(\epsilon_{1}, \epsilon_{2}\right)$ associated to the $\mathrm{U}(1)$-isometry of $S^{1}$ and the $\mathrm{U}(1)$ isometry of $\mathbb{R}^{2} \subset \mathbb{R}^{3}$, which is only sypersymmetric if the ratio $b=\epsilon_{1} / \epsilon_{2}$ is fixed. To perform an analogous calculation for the $\frac{1}{2} \Omega$-background $\mathbb{R}_{\epsilon}^{2} \times \mathbb{R}^{2}$, one would need to consider the four supercharges preserved by the latter background (see Footnote 24).
} 
$(2,0)$-theory $\mathfrak{X}[K]$ on a three-manifold $M$. The three-manifolds $M$ that label the threedimensional theories $\mathrm{T}_{K}[M]$ of class $\mathcal{R}$ are assembled by gluing topological tetrahedra. This decomposition into tetrahedra determines an ideal triangulation $\mathscr{T}$ of their boundaries $\partial M$. The corresponding three-dimensional superconformal field theory depends on the triangulation $\mathscr{T}$ as well as a polarization $\Pi$ of the boundary, ${ }^{49}$ and is therefore often denoted as $\mathrm{T}_{K}[M, \mathscr{T}, \Pi]$. Although the discussion which follows can be generalized rather straightforwardly to any $K$ (with the help of $[40,60]$ ), we will often restrict ourselves here to $K=2$ for simplicity.

For $K=2$, we call an edge of the triangulation $\mathscr{T}$ electric or magnetic if the corresponding one-cycle $\gamma \in H_{1}(\bar{\Sigma}, \mathbb{Z})$ is an $A$-cycle or a $B$-cycle, respectively. Each electric edge corresponds to a manifest $\mathrm{U}(1)$ flavour symmetry in the three-dimensional superconformal field theory. For every such edge $E$ there is a chiral operator $\mathcal{O}_{E}$ which transforms with charge +1 under the corresponding $U(1)$ flavour symmetry. If $E$ instead has a nontrivial magnetic charge, then an operator $\mathcal{O}_{E}$ exists in the presence of a magnetic monopole background.

Given a four-dimensional $\mathcal{N}=2$ theory $\mathrm{T}_{K}[C]$ in an infrared vacuum $u$, labelled by a (possibly) punctured Riemann surface $C$, we constructed the "empty" boundary condition

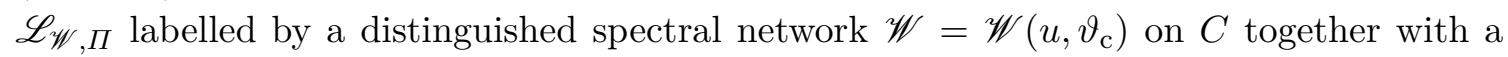
polarization $\Pi$ of the Seiberg-Witten curve $\Sigma_{u}$, which is given by the equations $\mathcal{X}_{B_{i}}^{\mathscr{W}}=1$. It should now be clear that we may generalize this boundary condition to a "full" boundary condition $\mathscr{L}_{M, \mathscr{W}^{\prime}, \Pi^{\prime}}$ labelled by a three-manifold $M$ (assembled as above by gluing tetrahedra) with boundary $\partial M=C \sqcup C^{\prime}$, whose boundary triangulation $\mathscr{T} \sqcup \mathscr{T}^{\prime}$ is dual to the pair of spectral networks $\mathscr{W} \sqcup \mathscr{W}^{\prime}$ on $C \sqcup C^{\prime}$, and with boundary polarizations $\Pi$ on $C$ and $\Pi^{\prime}$ on $C^{\prime}$. The new boundary condition $\mathscr{L}_{M, \mathscr{W}^{\prime}, \Pi^{\prime}}$ is defined by the equations

$$
\mathcal{X}_{B_{i}^{\prime}}^{\mathscr{W}^{\prime}}=1
$$

in the system of exponentiated Darboux coordinates $\left\{\mathcal{X}_{A^{\prime}}^{\mathscr{W}^{\prime}}, \mathcal{X}_{B_{i}^{\prime}}^{\mathscr{W}^{\prime}}\right\}$ obtained by abelianization with respect to the spectral network $\mathscr{W}^{\prime}$.

In the field theory description when $K=2$, the abelian gauge fields of the fourdimensional theory $\mathrm{T}_{2}[C]$ in the vacuum $u$ can be used to gauge the flavour symmetries of the three-dimensional theory $\mathrm{T}_{2}[M]$ corresponding to the edges $E$ of the triangulation $\mathscr{T}$, whereas the electric BPS hypermultiplets of $\mathrm{T}_{2}[C]$ may be coupled to the corresponding chiral operators $\mathcal{O}_{E}$ of $\mathrm{T}_{2}[M]$. This eliminates the dependence of the resulting fourdimensional theory on $\mathscr{T}$ and $\Pi$. A similar argument can be made for arbitrary $K$.

The simplest new boundary conditions are those that leave the vacuum $u$ invariant. Let us examine two types of examples. First, consider a boundary condition $\mathscr{L}_{M, \mathscr{W}, \Pi^{\prime}}$ where only the polarization $\Pi \rightarrow \Pi^{\prime}$ of the Seiberg-Witten curve $\Sigma_{u}$ is modified. This change corresponds to an electric-magnetic duality transformation $g \in \operatorname{Sp}(2 r, \mathbb{Z})$ in the infrared vacuum $u$, which is implemented by a generalized Legendre transform $\mathcal{L}_{\mathrm{g}}$ at the level of the effective twisted superpotential, associated to the linear symplectomorphism $\left(\begin{array}{l}x \\ y\end{array}\right) \mapsto\left(\begin{array}{l}x^{\prime} \\ y^{\prime}\end{array}\right)=\mathrm{g}\left(\begin{array}{l}x \\ y\end{array}\right)$ (see e.g. [138]). For the elementary canonical transformation

\footnotetext{
${ }^{49}$ More precisely, $\Pi$ is a polarization for an open subset of the moduli space $\mathscr{M}_{\text {flat }}(\partial M, \mathrm{SL}(K, \mathbb{C}))$ of framed flat $\operatorname{SL}(K, \mathbb{C})$ connections on $\partial M$.
} 


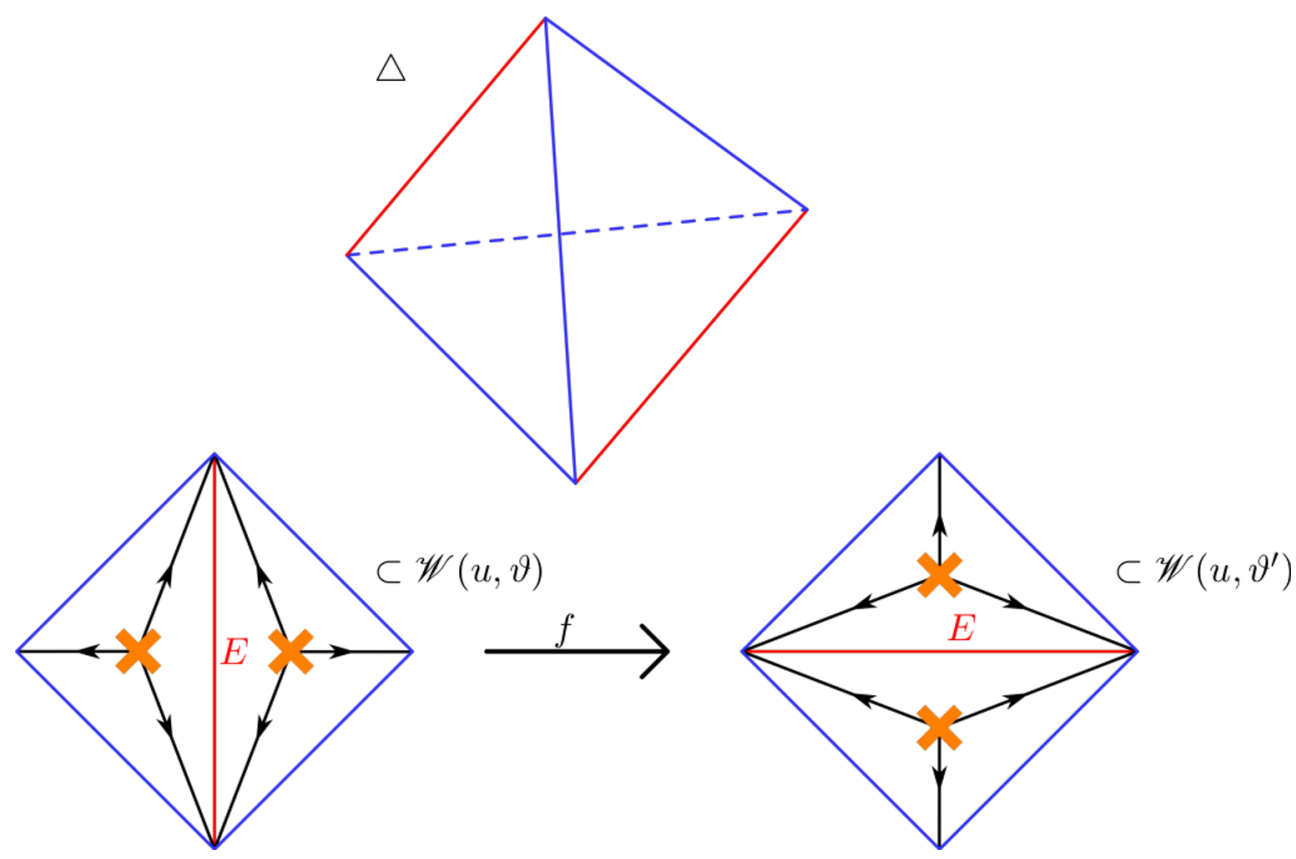

Figure 42. Each flip $f$ in the transition from $\mathscr{W}(u, \vartheta)$ to $\mathscr{W}\left(u, \vartheta^{\prime}\right)$ corresponds to a tetrahedron $\triangle$ in $M$, where the triangles adjacent to the flipped edge $E$ form the faces of $\triangle$.

$\left(\begin{array}{l}x^{\prime} \\ y^{\prime}\end{array}\right)=\mathrm{g}_{0}\left(\begin{array}{l}x \\ y\end{array}\right)=\left(\begin{array}{c}-y \\ x\end{array}\right)$, this Legendre transform has the familiar classical expression

$$
\mathcal{L}_{\mathrm{g}_{0}}: \widetilde{\mathcal{W}}^{\mathrm{eff}}(x ; \epsilon) \longmapsto \widetilde{\mathcal{W}}^{\text {eff }}\left(x^{\prime} ; \epsilon\right)=\epsilon x^{\prime} \cdot x+\widetilde{\mathcal{W}}^{\mathrm{eff}}(x ; \epsilon) .
$$

We may also simply change the boundary triangulation $\mathscr{T} \rightarrow \mathscr{T}^{\prime}$ on the second copy $C^{\prime}=C$ of the boundary $\partial M=C \sqcup C^{\prime}$. Let $K=2$. Assume that $\mathscr{T}$ and $\mathscr{T}^{\prime}$ are dual to the spectral networks $\mathscr{W}=\mathscr{W}(u, \vartheta)$ and $\mathscr{W}^{\prime}=\mathscr{W}\left(u, \vartheta^{\prime}\right)$, respectively, and that $\mathscr{W}(u, \vartheta)$ and $\mathscr{W}\left(u, \vartheta^{\prime}\right)$ are related by a sequence of flips. As illustrated in figure 42, each flip in the transition from $\mathscr{W}(u, \vartheta)$ to $\mathscr{W}\left(u, \vartheta^{\prime}\right)$ corresponds to a tetrahedron in the decomposition of $M$ into tetrahedra. The coordinate change corresponding to such a flip is given by

$$
\mathcal{X}_{\gamma_{E}}^{\mathscr{W}^{\prime}}=\mathcal{X}_{\gamma_{E}}^{\mathscr{W}} \quad \text { and } \quad \mathcal{X}_{\gamma}^{\mathscr{W}^{\prime}}=\mathcal{X}_{\gamma}^{\mathscr{W}}\left(1+\mathcal{X}_{\gamma_{E}}^{\mathscr{W}}\right)^{\left\langle\gamma, \gamma_{E}\right\rangle},
$$

where $\gamma_{E}$ is the one-cycle on $\Sigma_{u}$ corresponding to the edge $E$ of $\mathscr{T}$ in figure 42 , and $\gamma$ is any other one-cycle on $\Sigma_{u}$ (see [59, section 7.6]). Let $\gamma$ be any one-cycle with $\left\langle\gamma, \gamma_{E}\right\rangle=1$, and consider the Darboux coordinates $x=\frac{\epsilon}{\pi \mathrm{i}} \log \left(-\mathcal{X}_{\gamma_{E}}^{\mathscr{W}}\right), y=\frac{1}{2 \epsilon} \log \mathcal{X}_{\gamma}^{\mathscr{W}}$ and $y^{\prime}=\frac{1}{2 \epsilon} \log \mathcal{X}_{\gamma}^{\mathscr{W}^{\prime}}$. The effective twisted superpotential then changes by

$$
\begin{aligned}
\widetilde{\mathcal{W}}^{\prime \text { eff }}(x ; \epsilon)-\widetilde{\mathcal{W}}^{\text {eff }}(x ; \epsilon) & =\frac{1}{2} \int\left(\log \mathcal{X}_{\gamma}^{\mathscr{W}^{\prime}}-\log \mathcal{X}_{\gamma}^{\mathscr{W}}\right) \mathrm{d} x \\
& =\frac{1}{2} \int \log \left(1-\mathrm{e}^{\pi \mathrm{i} x / \epsilon}\right) \mathrm{d} x \\
& =-\frac{\epsilon}{2 \pi \mathrm{i}} \operatorname{Li}_{2}\left(\mathrm{e}^{\pi \mathrm{i} x / \epsilon}\right) .
\end{aligned}
$$

The difference (5.76) precisely matches the effective two-dimensional twisted superpotential $\widetilde{\mathcal{W}}_{\triangle}^{\text {eff }}(x ; \epsilon)$ for the elementary tetrahedron theory $\mathrm{T}_{2}\left[\triangle, \mathscr{T}, \Pi_{E}\right]$ in the background 
$\widetilde{S}^{1} \times \mathbb{R}^{2}$ (the boundary of $D_{R, \epsilon} \times \mathbb{R}^{2}$ ). Indeed, the theory $\mathrm{T}_{2}\left[\triangle, \mathscr{T}, \Pi_{E}\right]$ consists of a single free chiral multiplet $\phi_{E}$ with charge +1 under the $\mathrm{U}(1)$ flavour symmetry associated to the edge $E$, charge 0 under the $\mathrm{U}(1)_{R}$ symmetry, and a level $-\frac{1}{2}$ background Chern-Simons term for the difference of the corresponding gauge fields $A_{\mathrm{f}}-A_{R}$. The result (5.76) is the one-loop contribution of the chiral field $\phi_{E}$ with effective twisted mass $\pi \mathrm{i} x / \epsilon$ to the effective twisted superpotential, which is obtained as a sum over its Kaluza-Klein modes in the compactification to $\mathbb{R}^{2}$ [140]. Geometrically it corresponds to the hyperbolic volume of the tetrahedron $\triangle$.

Implementing the modifications to the four-dimensional effective twisted superpotential for a sequence of flips might require us to adapt the polarization on $\Sigma_{u}$ along the way. This has the effect of an affine symplectic transformation on the three-dimensional $\mathcal{N}=2$ theory $\mathrm{T}_{2}[M]$ (see [143] and [40, appendix A]): "T-type" transformations add background Chern-Simons couplings for flavour symmetries, while "S-type" transformations gauge a flavour symmetry, replacing it with a new topological $\mathrm{U}(1)_{J}$ symmetry.

This type of generalized boundary condition does not depend on the complex structure of $C$ and hence can only change the one-loop part of the effective twisted superpotential $\widetilde{\mathcal{W}}^{\text {eff }}(x ; \epsilon)$. Considering the effective twisted superpotential with respect to the spectral coordinates $\left\{\mathcal{X}_{A}, \mathcal{X}_{B}^{ \pm}\right\}$corresponding to a resolution of a Fenchel-Nielsen type network, as we did at the end of section 5.4, is another example of such a generalized boundary condition. Recall that for these choices, we found that the resulting one-loop contribution agrees with the one-loop contribution to $\widetilde{\mathcal{W}}^{\text {eff }}(x ; \epsilon)$ in the gauge theory or topological string scheme.

So far we have studied two types of more general infrared boundary conditions for the four-dimensional theory $\mathrm{T}_{K}[C]$ in the vacuum $u$. By choosing more complicated threemanifolds $M$, we may however also engineer three-dimensional boundary conditions for the theory $\mathrm{T}_{K}[C]$ in a different vacuum $u^{\prime}$ (because of the $\operatorname{Sp}(2 r, \mathbb{Z})$ monodromies on the Coulomb branch $\mathscr{B}$, the three-manifold $M$ as well as the resulting effective twisted superpotential will depend on a choice of path from $u$ to $u^{\prime}$ on $\mathscr{B}$ ). In the language of [43] this means that we should consider three-manifolds with not only non-trivial "big" boundary components, but also with non-trivial "small" boundary components, corresponding to annuli or tori that stem from truncating the tetrahedra in the decomposition of $M .^{50}$

While we do not describe such boundary conditions in detail here, these considerations do motivate the definition of a new object: the (generalized) effective twisted superpotential $\widetilde{\mathcal{W}}_{u, \vartheta, \Pi}^{\text {eff }}(x ; \epsilon)$. We define this twisted superpotential as the generating function of the brane of $\epsilon$-opers in the spectral coordinates $\left\{\mathcal{X}_{A^{i}}^{\mathscr{W}(u, \vartheta)}, \mathcal{X}_{B_{i}}^{\mathscr{W}(u, \vartheta)}\right\}$ obtained from abelianization with respect to any spectral network $\mathscr{W}(u, \vartheta)$, determined by $u$ and $\vartheta$, as well as the polarization

\footnotetext{
${ }^{50}$ More accurately, the empty boundary condition $\mathscr{L}_{\mathscr{W}, \Pi}$ can be constructed, according to [43], as a combination of a UV-IR domain wall and an infrared boundary condition. This combination corresponds geometrically to a three-manifold $M$ with two boundary components $C$ and $\bar{C}$. Here $C$ is a "big" boundary component together with a triangulation $\mathscr{T}$ dual to $\mathscr{W}$ and polarization $\Pi$, while $\bar{C}$ is only homeomorphic to $C$ and consists of "big" three-punctured spheres glued together with "small" annuli. The more general boundary conditions discussed here may then be constructed as a combination of an S-duality wall (which connects $C$ with complex structure $q$ to $C^{\prime}$ with complex structure $q^{\prime}$ ), a UV-IR domain wall (which flows the theory to the vacuum $u^{\prime}$ ) as well as an infrared boundary condition (determined by the spectral network $\mathscr{W}^{\prime}$ and polarization $\Pi^{\prime}$ on $C^{\prime}$ ).
} 
$\Pi$ on the Seiberg-Witten curve $\Sigma_{u}$. That is, the effective twisted superpotential is extracted from the relations

$$
\begin{aligned}
\log \left(-\mathcal{X}_{A^{i}}^{\mathscr{W}(u, \vartheta)}\left(\nabla_{\epsilon}^{\text {oper }}\right)\right) & =\frac{\pi \mathrm{i} x_{i}}{\epsilon}, \\
\log \mathcal{X}_{B_{i}}^{\mathscr{W}(u, \vartheta)}\left(\nabla_{\epsilon}^{\text {oper }}\right) & =2 \frac{\partial \widetilde{\mathcal{W}}_{u, \vartheta, \Pi}^{\text {eff }}}{\partial x_{i}} .
\end{aligned}
$$

Physically it corresponds to the effective twisted superpotential of the theory $\mathrm{T}_{K}[C]$ in the

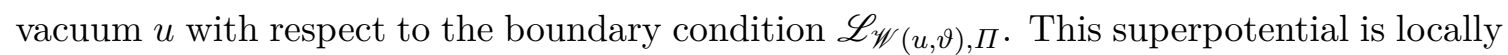
constant on $\mathscr{B} \times[0,2 \pi)$, and it is analytic in $x$ and $\epsilon$ for fixed $(u, \vartheta)$. It is defined uniquely on $\mathscr{B} \times[0,2 \pi)$ up to the monodromies of the $\operatorname{Sp}(2 r, \mathbb{Z})$ bundle over the Coulomb branch $\mathscr{B}$.

Open Access. This article is distributed under the terms of the Creative Commons Attribution License (CC-BY 4.0), which permits any use, distribution and reproduction in any medium, provided the original author(s) and source are credited.

\section{References}

[1] M. Abramowitz and I.A. Stegun, Handbook of mathematical functions, Dover Publications, U.S.A. (1965).

[2] M. Aganagic, N. Haouzi, C. Kozcaz and S. Shakirov, Gauge/Liouville triality, arXiv: 1309.1687 [INSPIRE].

[3] M. Aganagic, N. Haouzi and S. Shakirov, $A_{n}$-triality, arXiv:1403.3657 [INSPIRE].

[4] L.F. Alday, D. Gaiotto, S. Gukov, Y. Tachikawa and H. Verlinde, Loop and surface operators in $N=2$ gauge theory and Liouville modular geometry, JHEP 01 (2010) 113 [arXiv:0909.0945] [INSPIRE].

[5] L.F. Alday, D. Gaiotto and Y. Tachikawa, Liouville correlation functions from four-dimensional gauge theories, Lett. Math. Phys. 91 (2010) 167 [arXiv:0906.3219] [INSPIRE].

[6] M. Alim, Difference equation for the Gromov-Witten potential of the resolved conifold, arXiv:2011.12759 [INSPIRE].

[7] M. Alim, Intrinsic non-perturbative topological strings, arXiv:2102.07776 [INSPIRE].

[8] M. Alim, S. Cecotti, C. Cordova, S. Espahbodi, A. Rastogi and C. Vafa, BPS quivers and spectra of complete $N=2$ quantum field theories, Commun. Math. Phys. 323 (2013) 1185 [arXiv:1109.4941] [INSPIRE].

[9] M. Alim, A. Saha, J. Teschner and I. Tulli, Mathematical structures of non-perturbative topological string theory: from GW to DT invariants, arXiv:2109.06878 [INSPIRE].

[10] D.G.L. Allegretti, Voros symbols as cluster coordinates, J. Topol. 12 (2019) 1031 [arXiv: 1802 . 05479] [INSPIRE].

[11] D.G.L. Allegretti, Stability conditions, cluster varieties, and Riemann-Hilbert problems from surfaces, Adv. Math. 380 (2021) 107610 [arXiv: 1912.05938] [INSPIRE].

[12] P.C. Argyres and M.R. Douglas, New phenomena in SU(3) supersymmetric gauge theory, Nucl. Phys. B 448 (1995) 93 [hep-th/9505062] [INSPIRE]. 
[13] P.C. Argyres and N. Seiberg, S-duality in $N=2$ supersymmetric gauge theories, JHEP 12 (2007) 088 [arXiv:0711.0054] [INSPIRE].

[14] S.K. Ashok, M. Billó, E. Dell'Aquila, M. Frau, R.R. John and A. Lerda, Non-perturbative studies of $N=2$ conformal quiver gauge theories, Fortsch. Phys. 63 (2015) 259 [arXiv: 1502.05581] [INSPIRE].

[15] M.F. Atiyah and R. Bott, The Yang-Mills equations over Riemann surfaces, Phil. Trans. Roy. Soc. London A 308 (1982) 523.

[16] T. Banks, M.R. Douglas and N. Seiberg, Probing F-theory with branes, Phys. Lett. B 387 (1996) 278 [hep-th/9605199] [INSPIRE].

[17] L. Bao, V. Mitev, E. Pomoni, M. Taki and F. Yagi, Non-Lagrangian theories from brane junctions, JHEP 01 (2014) 175 [arXiv:1310.3841] [INSPIRE].

[18] A. Barbieri, T. Bridgeland and J. Stoppa, A quantized Riemann-Hilbert problem in Donaldson-Thomas theory, arXiv: 1905.00748 [INSPIRE].

[19] A. Beilinson and V. Drinfeld, Opers, math.AG/0501398.

[20] M. Bershadsky, A. Johansen, V. Sadov and C. Vafa, Topological reduction of 4D SYM to 2D sigma models, Nucl. Phys. B 448 (1995) 166 [hep-th/9501096] [INSPIRE].

[21] P. Boalch and O. Biquard, Wild non-abelian Hodge theory on curves, Compos. Math. 140 (2004) 179 [math.DG/0111098].

[22] P. Boalch, Geometry and braiding of Stokes data; Fission and wild character varieties, Ann. Math. 179 (2014) 301.

[23] G. Bonelli, O. Lisovyy, K. Maruyoshi, A. Sciarappa and A. Tanzini, On Painlevé/gauge theory correspondence, Lett. Math. Phys. 107 (2017) pages 2359 [arXiv:1612.06235] [INSPIRE].

[24] T.D. Brennan and G.W. Moore, Index-like theorems from line defect vevs, JHEP 09 (2019) 073 [arXiv: 1903.08172] [INSPIRE].

[25] T. Bridgeland, Riemann-Hilbert problems from Donaldson-Thomas theory, Invent. Math. 216 (2019) 69 [arXiv:1611.03697] [INSPIRE].

[26] T. Bridgeland, Riemann-Hilbert problems for the resolved conifold, J. Diff. Geom. 115 (2020) 395 [arXiv:1703.02776] [INSPIRE].

[27] T. Bridgeland and D. Masoero, On the monodromy of the deformed cubic oscillator, arXiv:2006.10648.

[28] M. Bullimore, T. Dimofte, D. Gaiotto and J. Hilburn, Boundaries, mirror symmetry, and symplectic duality in $3 d \mathcal{N}=4$ gauge theory, JHEP 10 (2016) 108 [arXiv:1603.08382] [INSPIRE].

[29] S. Cecotti, C. Cordova and C. Vafa, Braids, walls, and mirrors, arXiv:1110.2115 [INSPIRE].

[30] S. Cecotti and C. Vafa, Classification of complete $N=2$ supersymmetric theories in 4 dimensions, J. Diff. Geom. 18 (2013) 19 [arXiv:1103.5832] [InSPIRE].

[31] O. Chacaltana and J. Distler, Tinkertoys for Gaiotto duality, JHEP 11 (2010) 099 [arXiv: 1008.5203] [INSPIRE]. 
[32] S.A. Cherkis and A. Kapustin, Nahm transform for periodic monopoles and $N=2$ super-Yang-Mills theory, Commun. Math. Phys. 218 (2001) 333 [hep-th/0006050] [INSPIRE].

[33] P. Claus, R. Kallosh and A. Van Proeyen, $M$ five-brane and superconformal $(0,2)$ tensor multiplet in six-dimensions, Nucl. Phys. B 518 (1998) 117 [hep-th/9711161] [INSPIRE].

[34] S. Codesido, A. Grassi and M. Mariño, Spectral theory and mirror curves of higher genus, Ann. H. Poincaré 18 (2017) 559 [arXiv:1507.02096] [InSPIRE].

[35] I. Coman, P. Longhi and J. Teschner, From quantum curves to topological string partition functions II, arXiv:2004.04585 [INSPIRE].

[36] I. Coman, E. Pomoni and J. Teschner, From quantum curves to topological string partition functions, arXiv:1811.01978 [INSPIRE].

[37] I. Coman, E. Pomoni and J. Teschner, Toda conformal blocks, quantum groups, and flat connections, Commun. Math. Phys. 375 (2019) 1117 [arXiv:1712.10225] [INSPIRE].

[38] I. Coman, E. Pomoni and J. Teschner, Trinion conformal blocks from topological strings, JHEP 09 (2020) 078 [arXiv: 1906.06351] [INSPIRE].

[39] R. Dijkgraaf, Les Houches lectures on fields, strings and duality, in NATO Advanced Study Institute: Les Houches Summer School on Theoretical Physics, Session 64: Quantum Symmetries, August 1-September 8, Les Houches, France (1997) [hep-th/9703136] [INSPIRE].

[40] T. Dimofte, M. Gabella and A.B. Goncharov, K-decompositions and $3 d$ gauge theories, JHEP 11 (2016) 151 [arXiv:1301.0192] [InSPIRE].

[41] T. Dimofte, D. Gaiotto and S. Gukov, 3-manifolds and 3d indices, Adv. Theor. Math. Phys. 17 (2013) 975 [arXiv: 1112.5179] [INSPIRE].

[42] T. Dimofte, D. Gaiotto and S. Gukov, Gauge theories labelled by three-manifolds, Commun. Math. Phys. 325 (2014) 367 [arXiv:1108.4389] [INSPIRE].

[43] T. Dimofte, D. Gaiotto and R. van der Veen, $R G$ domain walls and hybrid triangulations, Adv. Theor. Math. Phys. 19 (2015) 137 [arXiv:1304.6721] [INSPIRE].

[44] R. Donagi and E. Witten, Supersymmetric Yang-Mills theory and integrable systems, Nucl. Phys. B 460 (1996) 299 [hep-th/9510101] [INSPIRE].

[45] S.K. Donaldson, A new proof of a theorem of Narasimhan and Seshadri, J. Diff. Geom. 18 (1983) 269.

[46] N. Drukker, J. Gomis, T. Okuda and J. Teschner, Gauge theory loop operators and Liouville theory, JHEP 02 (2010) 057 [arXiv:0909.1105] [INSPIRE].

[47] N. Drukker, D.R. Morrison and T. Okuda, Loop operators and S-duality from curves on Riemann surfaces, JHEP 09 (2009) 031 [arXiv:0907.2593] [INSPIRE].

[48] O. Dumitrescu, A journey from the Hitchin section to the oper moduli, Proc. Symp. Pure Math. 98 (2018) 107 [arXiv: 1701.00155] [INSPIRE].

[49] O. Dumitrescu et al., Opers versus non-Abelian Hodge, J. Diff. Geom. 117 (2021) 223 [arXiv: 1607. 02172] [INSPIRE].

[50] B. Feigin and E. Frenkel, Quantization of soliton systems and Langlands duality, arXiv:0705.2486 [INSPIRE]. 
[51] E. Frenkel, Lectures on the Langlands program and conformal field theory, in Les Houches School of Physics: Frontiers in Number Theory, Physics and Geometry, July 2-27, Les Houches, France (2007) [hep-th/0512172] [INSPIRE].

[52] M. Gabella, P. Longhi, C.Y. Park and M. Yamazaki, BPS graphs: from spectral networks to BPS quivers, JHEP 07 (2017) 032 [arXiv: 1704.04204] [INSPIRE].

[53] A. Gadde, S.S. Razamat and B. Willett, "Lagrangian" for a non-Lagrangian field theory with $\mathcal{N}=2$ supersymmetry, Phys. Rev. Lett. 115 (2015) 171604 [arXiv:1505.05834] [iNSPIRE].

[54] D. Gaiotto, $N=2$ dualities, JHEP 08 (2012) 034 [arXiv:0904.2715] [InSPIRE].

[55] D. Gaiotto, Opers and TBA, arXiv:1403.6137 [INSPIRE].

[56] D. Gaiotto, G.W. Moore and A. Neitzke, Four-dimensional wall-crossing via three-dimensional field theory, Commun. Math. Phys. 299 (2010) 163 [arXiv:0807.4723] [INSPIRE].

[57] D. Gaiotto, G.W. Moore and A. Neitzke, Framed BPS states, Adv. Theor. Math. Phys. 17 (2013) 241 [arXiv: 1006.0146] [INSPIRE].

[58] D. Gaiotto, G.W. Moore and A. Neitzke, Spectral networks, Ann. H. Poincaré 14 (2013) 1643 [arXiv: 1204.4824] [INSPIRE].

[59] D. Gaiotto, G.W. Moore and A. Neitzke, Wall-crossing, Hitchin Systems, and the WKB Approximation, Adv. Math. 234 (2013) 239 [arXiv:0907.3987] [INSPIRE].

[60] D. Gaiotto, G.W. Moore and A. Neitzke, Spectral networks and snakes, Ann. H. Poincaré 15 (2014) 61 [arXiv:1209.0866] [InSPIRE].

[61] D. Galakhov, P. Longhi, T. Mainiero, G.W. Moore and A. Neitzke, Wild wall crossing and BPS giants, JHEP 11 (2013) 046 [arXiv: 1305.5454] [INSPIRE].

[62] D. Galakhov, P. Longhi and G.W. Moore, Spectral networks with spin, Commun. Math. Phys. 340 (2015) 171 [arXiv:1408.0207] [INSPIRE].

[63] W.M. Goldman, The symplectic nature of fundamental groups of surfaces, Adv. Math. 54 (1984) 200.

[64] A. Gorsky, I. Krichever, A. Marshakov, A. Mironov and A. Morozov, Integrability and Seiberg-Witten exact solution, Phys. Lett. B 355 (1995) 466 [hep-th/9505035] [InSPIRE].

[65] A. Grassi, J. Gu and M. Mariño, Non-perturbative approaches to the quantum Seiberg-Witten curve, JHEP 07 (2020) 106 [arXiv: 1908. 07065] [INSPIRE].

[66] A. Grassi, Q. Hao and A. Neitzke, Exact WKB methods in $\mathrm{SU}(2) N_{f}=1$, arXiv:2105.03777 [INSPIRE].

[67] A. Grassi, Y. Hatsuda and M. Mariño, Topological strings from quantum mechanics, Ann. H. Poincaré 17 (2016) 3177 [arXiv:1410.3382] [INSPIRE].

[68] R.C. Gunning, Special coordinate coverings of Riemann surfaces, Math. Ann. 170 (1967) 67.

[69] Q. Hao, L. Hollands and A. Neitzke, BPS states in the Minahan-Nemeschansky $E_{7}$ theory, JHEP 04 (2020) 039 [arXiv: 1905. 09879] [INSPIRE].

[70] J.A. Harvey, G.W. Moore and A. Strominger, Reducing S duality to T duality, Phys. Rev. D $\mathbf{5 2}$ (1995) 7161 [hep-th/9501022] [INSPIRE]. 
[71] N.J. Hitchin, The selfduality equations on a Riemann surface, Proc. Lond. Math. Soc. 55 (1987) 59 [INSPIRE].

[72] L. Hollands, C.A. Keller and J. Song, From SO/Sp instantons to W-algebra blocks, JHEP 03 (2011) 053 [arXiv: 1012.4468] [INSPIRE].

[73] L. Hollands, C.A. Keller and J. Song, Towards a 4d/2d correspondence for Sicilian quivers, JHEP 10 (2011) 100 [arXiv:1107.0973] [INSPIRE].

[74] L. Hollands and O. Kidwai, Higher length-twist coordinates, generalized Heun's opers, and twisted superpotentials, Adv. Theor. Math. Phys. 22 (2018) 1713 [arXiv:1710.04438] [INSPIRE].

[75] L. Hollands and A. Neitzke, Spectral networks and Fenchel-Nielsen coordinates, Lett. Math. Phys. 106 (2016) 811 [arXiv:1312.2979] [INSPIRE].

[76] L. Hollands and A. Neitzke, BPS states in the Minahan-Nemeschansky $E_{6}$ theory, Commun. Math. Phys. 353 (2017) 317 [arXiv: 1607.01743] [InSPIRE].

[77] L. Hollands and A. Neitzke, Exact $W K B$ and abelianization for the $T_{3}$ equation, Commun. Math. Phys. 380 (2020) 131 [arXiv:1906.04271] [InSPIRE].

[78] M.-x. Huang, A.-K. Kashani-Poor and A. Klemm, The $\Omega$ deformed B-model for rigid $\mathcal{N}=2$ theories, Annales Henri Poincaré 14 (2013) 425 [arXiv:1109.5728] [InSPIRE].

[79] M. Ionita and B. Morrissey, Spectral networks and non-abelianization, arXiv:2103.12285 [INSPIRE].

[80] K. Ito, M. Mariño and H. Shu, TBA equations and resurgent quantum mechanics, JHEP 01 (2019) 228 [arXiv: 1811.04812] [INSPIRE].

[81] Y. Ito, T. Okuda and M. Taki, Line operators on $S^{1} \times \mathbb{R}^{3}$ and quantization of the Hitchin moduli space, JHEP 04 (2012) 010 [Erratum ibid. 03 (2016) 085] [arXiv:1111.4221] [INSPIRE].

[82] K. Iwaki and O. Kidwai, Topological recursion and uncoupled BPS structures I: BPS spectrum and free energies, arXiv:2010.05596 [INSPIRE].

[83] K. Iwaki and O. Kidwai, Topological recursion and uncoupled BPS structures II: Voros symbols and the $\tau$-function, arXiv:2108.06995 [INSPIRE].

[84] K. Iwaki and T. Nakanishi, Exact WKB analysis and cluster algebras, J. Phys. A 47 (2014) 474009 .

[85] S. Jeong and N. Nekrasov, Opers, surface defects, and Yang-Yang functional, Adv. Theor. Math. Phys. 24 (2020) 1789 [arXiv:1806.08270] [InSPIRE].

[86] S. Jeong and N. Nekrasov, Riemann-Hilbert correspondence and blown up surface defects, JHEP 12 (2020) 006 [arXiv:2007.03660] [INSPIRE].

[87] A. Kapustin, L. Rozansky and N. Saulina, Three-dimensional topological field theory and symplectic algebraic geometry I, Nucl. Phys. B 816 (2009) 295 [arXiv:0810.5415] [INSPIRE].

[88] A. Kapustin and E. Witten, Electric-magnetic duality and the geometric Langlands program, Commun. Num. Theor. Phys. 1 (2007) 1 [hep-th/0604151] [INSPIRE].

[89] T. Kawai and Y. Takei, Algebraic analysis of singular perturbation theory, Maerican Mathematical Society, U.S.A. (2005). 
[90] A. Klemm, W. Lerche, P. Mayr, C. Vafa and N.P. Warner, Selfdual strings and $N=2$ supersymmetric field theory, Nucl. Phys. B 477 (1996) 746 [hep-th/9604034] [INSPIRE].

[91] T. Koike and R. Schäfke, On the Borel summability of WKB solutions of Schrödinger equations with polynomial potentials and its applications, to appear.

[92] H. Konno, Construction of the moduli space of stable parabolic Higgs bundles on a Riemann surface, J. Math. Soc. Japan 45 (1993) 253.

[93] M. Kontsevich, Intersection theory on the moduli space of curves and the matrix Airy function, Comm. Math. Phys. 147 (1992) 1.

[94] M. Kontsevich and Y. Soibelman, Stability structures, motivic Donaldson-Thomas invariants and cluster transformations, arXiv:0811.2435 [INSPIRE].

[95] W. Lerche, Introduction to Seiberg-Witten theory and its stringy origin, Nucl. Phys. B Proc. Suppl. 55 (1997) 83 [hep-th/9611190] [INSPIRE].

[96] P. Longhi, Wall-crossing invariants from spectral networks, Ann. H. Poincaré 19 (2018) 775 [arXiv: 1611.00150] [INSPIRE].

[97] P. Longhi and C.Y. Park, ADE spectral networks, JHEP 08 (2016) 087 [arXiv: 1601.02633] [INSPIRE].

[98] M. Martone, The constraining power of Coulomb Branch Geometry: lectures on Seiberg-Witten theory, arXiv:2006.14038 [INSPIRE].

[99] K. Maruyoshi and J. Song, Enhancement of supersymmetry via renormalization group flow and the superconformal index, Phys. Rev. Lett. 118 (2017) 151602 [arXiv:1606.05632] [INSPIRE].

[100] M. Matone, Instantons and recursion relations in $N=2$ SUSY gauge theory, Phys. Lett. B 357 (1995) 342 [hep-th/9506102] [INSPIRE].

[101] J.A. Minahan and D. Nemeschansky, An $N=2$ superconformal fixed point with $E_{6}$ global symmetry, Nucl. Phys. B 482 (1996) 142 [hep-th/9608047] [INSPIRE].

[102] A. Mironov and A. Morozov, Nekrasov functions and exact Bohr-Zommerfeld integrals, JHEP 04 (2010) 040 [arXiv:0910.5670] [InSPIRE].

[103] V. Mitev and E. Pomoni, Toda 3-point functions from topological strings, JHEP 06 (2015) 049 [arXiv: 1409.6313] [INSPIRE].

[104] V. Mitev, E. Pomoni, M. Taki and F. Yagi, Fiber-base duality and global symmetry enhancement, JHEP 04 (2015) 052 [arXiv:1411.2450] [INSPIRE].

[105] G.W. Moore, PiTP lectures on BPS states and wall-crossing in $d=4, \mathcal{N}=2$ theories, http://www.physics.rutgers.edu/ gmoore/PiTP-LectureNotes.pdf.

[106] G.W. Moore, Applications of the six-dimensional (2,0)-theory to physical mathematics, http://www.physics.rutgers.edu/ gmoore/FelixKleinLectureNotes.pdf.

[107] W. Nahm, Supersymmetries and their representations, Nucl. Phys. B 135 (1978) 149 [INSPIRE].

[108] H. Nakajima, Hyperkähler structures on moduli spaces of parabolic Higgs bundles on Riemann surfaces, Lect. Notes Pure Appl. Math. 179 (1996) 199.

[109] A. Neitzke, Hitchin systems in $\mathcal{N}=2$ field theory, in New dualities of supersymmetric gauge theories, J. Teschner ed., Springer, Germany (2016) [arXiv:1412.7120] [INSPIRE]. 
[110] A. Neitzke, swn-plotter, Mathematica notebook, https://gauss.math.yale.edu/ an592/.

[111] N.A. Nekrasov, Seiberg-Witten prepotential from instanton counting, Adv. Theor. Math. Phys. 7 (2003) 831 [hep-th/0206161] [INSPIRE].

[112] N. Nekrasov, Blowups in BPS/CFT correspondence, and Painlevé VI, arXiv:2007.03646 [INSPIRE].

[113] N. Nekrasov and V. Pestun, Seiberg-Witten geometry of four dimensional $N=2$ quiver gauge theories, arXiv: 1211.2240 [INSPIRE].

[114] N. Nekrasov, A. Rosly and S. Shatashvili, Darboux coordinates, Yang-Yang functional, and gauge theory, Nucl. Phys. B Proc. Suppl. 216 (2011) 69 [arXiv:1103.3919] [InSPIRE].

[115] N.A. Nekrasov and S.L. Shatashvili, Quantization of integrable systems and four dimensional gauge theories, in the proceedings of the $16^{\text {th }}$ International Congress on Mathematical Physics, August 3-9, Prague, Czech Republic (2009) [arXiv:0908.4052] [INSPIRE].

[116] N. Nekrasov and E. Witten, The omega deformation, branes, integrability, and liouville theory, JHEP 09 (2010) 092 [arXiv: 1002.0888] [INSPIRE].

[117] N. Nikolaev, Exact solutions for the singularly perturbed Riccati equation and exact WKB analysis, arXiv:2008.06492.

[118] N. Nikolaev, Abelianisation of logarithmic $\mathfrak{s l}_{2}$-connections, Selecta Math. 27 (2021) 78 [arXiv: 1902.03384] [INSPIRE].

[119] T. Okuda, Line operators in supersymmetric gauge theories and the $2 d-4 d$ relation, in New dualities of supersymmetric gauge theories, J. Teschner ed., Springer, Germany (2016) [arXiv:1412.7126] [INSPIRE].

[120] F.J.W. Olver, D.W. Lozier, R.F. Boisvert and C.W. Clark, NIST Handbook of Mathematical Functions, Cambridge UNiversity Press, Cambridge U.K. (2010).

[121] V. Pestun, Localization of gauge theory on a four-sphere and supersymmetric Wilson loops, Commun. Math. Phys. 313 (2012) 71 [arXiv:0712.2824] [INSPIRE].

[122] V. Pestun et al., Localization techniques in quantum field theories, J. Phys. A 50 (2017) 440301 [arXiv: 1608.02952] [INSPIRE].

[123] N. Seiberg and E. Witten, Electric-magnetic duality, monopole condensation, and confinement in $N=2$ supersymmetric Yang-Mills theory, Nucl. Phys. B 426 (1994) 19 [Erratum ibid. 430 (1994) 485] [hep-th/9407087] [INSPIRE].

[124] N. Seiberg and E. Witten, Monopoles, duality and chiral symmetry breaking in $N=2$ supersymmetric QCD, Nucl. Phys. B 431 (1994) 484 [hep-th/9408099] [INSPIRE].

[125] N. Seiberg and E. Witten, Gauge dynamics and compactification to three-dimensions, in Mathematical beauty of physics, J.M. Drouffe and J.N. Zuber eds., World Scientific, Singapore (1997) [hep-th/9607163] [INSPIRE].

[126] C.T. Simpson, Higgs bundles and local systems, Publ. Math. IHÉS 75 (1992) 5.

[127] C.T. Simpson, The Hodge filtration on non-Abelian cohomology, Proc. Symp. Pure Math. 62 (1997) 217.

[128] J. Song, $4 d / 2 d$ correspondence: instantons and W-algebras, Ph.D. thesis, California Institute of Technology, U.S.A. (2012). 
[129] A. Strominger, Open p-branes, Phys. Lett. B 383 (1996) 44 [hep-th/9512059] [INSPIRE].

[130] R.J. Szabo, $N=2$ gauge theories, instanton moduli spaces and geometric representation theory, J. Geom. Phys. 109 (2016) 83 [arXiv:1507.00685] [InSPIRE].

[131] Y. Tachikawa, $N=2$ supersymmetric dynamics for pedestrians, Lecture Notes in Physics volume 890, Sprigner, Germany (2014) [arXiv: 1312.2684] [INSPIRE].

[132] Y. Tachikawa, A review of the $T_{N}$ theory and its cousins, PTEP 2015 (2015) $11 \mathrm{~B} 102$ [arXiv: 1504.01481] [INSPIRE].

[133] Y. Tachikawa, A review on instanton counting and $W$-algebras, in New dualities of supersymmetric gauge theories, J. Teschner ed., Springer, Germany (2016) [arXiv: 1412.7121] [INSPIRE].

[134] Y. Takei, WKB analysis and Stokes geometry of differential equations, in Analytic, algebraic and geometric aspects of differential equations, G. Filipuk et al. eds., Sprigner, Germany (2017).

[135] J. Teschner, New dualities of supersymmetric gauge theories, Mathematical Physics Studies, Springer, Germany (2016).

[136] J. Teschner, Quantisations of some spectral curves, Oberwolfach Rept. 9 (2016) 433.

[137] J. Teschner and G.S. Vartanov, Supersymmetric gauge theories, quantization of $\mathcal{M}_{\text {flat }}$, and conformal field theory, Adv. Theor. Math. Phys. 19 (2015) 1 [arXiv:1302.3778] [InSPIRE].

[138] W.M. Tulczyjew, The Legendre transformation, Ann.Inst.Henri Poincaré A 27 (1977) 101.

[139] E. Witten, Two-dimensional gravity and intersection theory on moduli space, Surv. Diff. Geom. 1 (1991) 243.

[140] E. Witten, Phases of $N=2$ theories in two-dimensions, Nucl. Phys. B 403 (1993) 159 [hep-th/9301042] [INSPIRE].

[141] E. Witten, Some comments on string dynamics, in Future Perspectives in String Theory, I. Bars et al. eds., World Scientific, Singapore (1995) [hep-th/9507121] [INSPIRE].

[142] E. Witten, Solutions of four-dimensional field theories via M-theory, Nucl. Phys. B 500 (1997) 3 [hep-th/9703166] [INSPIRE].

[143] E. Witten, $\operatorname{SL}(2, Z)$ action on three-dimensional conformal field theories with Abelian symmetry, in From fields to strings: circumnavigating theoretical physics, M. Shifman et al. eds., World Scientific, Singapore (2003) [hep-th/0307041] [INSPIRE].

[144] F. Yan, Exact WKB and the quantum Seiberg-Witten curve for $4 d N=2$ pure $S U(3)$ Yang-Mills, Part I: Abelianization, arXiv:2012.15658 [INSPIRE]. 